Pontifícia U Miversidade $C_{\text {atólica }}$

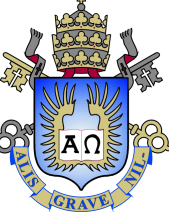

Alvaro Javier Ortega

\title{
On hybrid beamforming design for downlink mmWave massive MU-MIMO systems
}

\author{
Tese de Doutorado
}

Thesis presented to the Programa de Pós-graduação em Engenharia Elétrica of PUC-Rio in partial fulfillment of the requirements for the degree of Doutor em Engenharia Elétrica.

Advisor : Prof. Raimundo Sampaio-Neto Co-advisor: Dr. Rodrigo Pereira David 


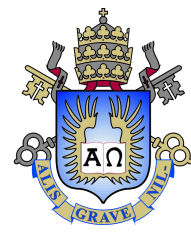

Alvaro Javier Ortega

\section{On hybrid beamforming design for downlink mmWave massive MU-MIMO systems}

Thesis presented to the Programa de Pós-graduação em Engenharia Elétrica of PUC-Rio in partial fulfillment of the requirements for the degree of Doutor em Engenharia Elétrica. Approved by the Examination Committee:

Prof. Raimundo Sampaio-Neto

Advisor

Centro de Estudos em Telecomunicações - PUC-Rio

Dr. Rodrigo Pereira David

Co-advisor

INMETRO

Dr. Ernesto Leite Pinto

IME

Dr. Marcello Luiz R. de Campos

COPPE/UFRJ

Dr. Paulo Sergio Ramirez Diniz

COPPE/UFRJ

Dr. João Alfredo Cal Braz

INMETRO

Rio de Janeiro, June the 1st 2020 
All rights reserved.

\section{Alvaro Javier Ortega}

Graduated in Electronic Engineering in 2013 at the University of Nariño, Nariño, Colombia. In 2016, he obtained a Master's degree in Electrical Engineering Sciences from the Pontifical Catholic University of Rio de Janeiro (PUC-Rio), Rio de Janeiro, Brazil, where, due to his outstanding academic performance he was granted a special scholarship Grade 10, FAPERJ. From February 2016 to July 2016, he worked as Lecturer at the University of Nariño, Nariño, Colombia. Since August 2016, he is a Ph.D. student at the Electrical Engineering Department of PUC-Rio, Rio de Janeiro, Brazil. Since August 2019, he is working at Sidia - Institute of Science and Technology, Manaus, Brazil

Bibliographic data

Ortega, Alvaro Javier

On hybrid beamforming design for downlink mmWave massive MU-MIMO systems / Alvaro Javier Ortega; advisor: Raimundo Sampaio-Neto; co-advisor: Rodrigo Pereira David. -2020 .

86 f. : il. color ; $30 \mathrm{~cm}$

Tese (doutorado) - Pontifícia Universidade Católica do Rio de Janeiro, Departamento de Engenharia Elétrica, 2020. Inclui bibliografia.

1. Engenharia Elétrica - Teses. 2. Ondas milimétricas. 3. Precodificação híbrida. 4. MIMO massivo. 5. Sistemas MIMO. 6. MIMO massivo multiusuário. I. Sampaio-Neto, Raimundo. II. Pereira David, Rodrigo. III. Pontifícia Universidade Católica do Rio de Janeiro. Departamento de Engenharia Elétrica. IV. Título. 


\section{Acknowledgments}

I am so grateful to my advisor, Raimundo Sampaio-Neto, for his support, sympathy and encouragement to carry out this work. He has provided me excellent guidance during my master and doctoral studies, greatly contributing to my formation.

Also, I want to thank to my co-advisor, Rodrigo Pereira David, who has guided this research, giving me immense support searching and studying papers with me, furthermore, solving my doubts every time that I needed.

Finally, I want to thank to the Brazilian Agency CNPq thas have supported this work. Also, this study was financed in part by the Coordenação de Aperfeiçoamento de Pessoal de Nível Superior - Brasil (CAPES) - Finance Code 001 


\section{Abstract}

Ortega, Alvaro Javier; Sampaio-Neto, Raimundo (Advisor); Pereira David, Rodrigo (Co-Advisor). On hybrid beamforming design for downlink mmWave massive MU-MIMO systems. Rio de Janeiro, 2020. 86p. Tese de Doutorado - Departamento de Engenharia Elétrica, Pontifícia Universidade Católica do Rio de Janeiro.

Millimeter-wave (mmWave) communications have been regarded as a key technology for the next-generation cellular systems since the huge available bandwidth can potentially provide the rates of multiple gigabits per second. Conventional precoding and combining techniques are impractical at mmWave scenarios due to manufacturing cost and power consumption. Hybrid alternatives have been considered as a promising technology to provide a compromise between hardware complexity and system performance. A large number of hybrid precoder designs have been proposed with different approaches. One possible approach is to search for minimizing the Euclidean distance between hybrid precoder and the full-digital precoder. However, this approach makes the hybrid precoder design becomes a matrix factorization problem difficult to deal due to the hardware constraints of analog components.

This doctoral thesis proposes some hybrid precoder and combiners designs through a hierarchical strategy. The hybrid precoding/combining problem is divided into analog and digital parts. First, the analog precoder/combiner is designed. Then, with the analog precoder/combiner fixed, the digital precoder/combiner is computed to improve the system performance. Furthermore, linear and no-linear optimization methods are employed to design the analog part of the precoder/combiner.

The viability of these proposals is evaluated using different data detection techniques and analyzing the system performance in terms of bit error rate (BER), sum rate, and other metrics, in indoor mmWave scenarios considering massive MU-MIMO downlink.

Also, this work proposes a method to find fairly tight analytic approximations to the obtained BER performance. The methodology proposed would require the knowledge of the probability density function (pdf) of the variables involved, which are unknown for mmWave scenarios. In order to solve 
this problem, Gamma pdf approximations are used. The analytic BER approximations resulted in differences no larger than $0.5 \mathrm{~dB}$ with respect to the simulation results in high SNR.

\section{Keywords}

mmWave; hybrid precoding; massive MIMO; MIMO Systems; multi-user massive MIMO.. 


\section{Resumo}

Ortega, Alvaro Javier; Sampaio-Neto, Raimundo (Advisor); Pereira David, Rodrigo (Co-Advisor). Projeto híbrido de formação de feixe para enlace direto em ondas milimétricas em sistemas massivos MU-MIMO. Rio de Janeiro, 2020. 86p. Tese de Doutorado - Departamento de Engenharia Elétrica, Pontifícia Universidade Católica do Rio de Janeiro.

As comunicações de ondas milimétricas (mmWave) são consideradas uma tecnologia essencial para os sistemas celulares de próxima geração, dado que a enorme largura de banda disponível pode potencialmente fornecer as taxas de vários gigabits por segundo. As técnicas convencionais de pré-codificação e combinação são impraticáveis nos cenários da mmWave devido ao custo de fabricação e ao consumo de energia. As alternativas híbridas foram consideradas uma tecnologia promissora para fornecer um compromisso entre a complexidade do hardware e o desempenho do sistema. Um grande número de projetos de pré-codificadores híbridos têm sido proposto com diferentes abordagens. Uma abordagem possível é procurar minimizar a distância euclidiana entre o pré-decodificador híbrido e o pré-decodificador totalmente digital. No entanto, essa abordagem torna o projeto do pré-codificador híbrido um problema de fatoração da matrices difícil de lidar devido às restrições de hardware dos componentes analógicos. Esta tese de doutorado propõe alguns projetos de pré-codificadores e combinadores híbridos por meio de uma estratégia hierárquica. O problema híbrido de pré-codificação / combinação é dividido em partes analógicas e digitais. Primeiro, o pré-codificador / combinador analógico é projetado. Em seguida, com o pré-codificador / combinador analógico fixo, o précodificador / combinador digital é calculado para melhorar o desempenho do sistema. Além disso, métodos de otimização linear e não linear são empregados para projetar a parte analógica do pré-codificador / combinador. A viabilidade dessas propostas é avaliada usando diferentes técnicas de detecção de dados e analisando o desempenho do sistema em termos de taxa de erros de bits (BER), sum-rate e outras métricas, em cenários internos do mmWave, considerando enlace diretos massivo do MU-MIMO.

Além disso, este trabalho propõe um método para encontrar aproximações analíticas bastante restritas ao desempenho obtido no BER. A metodologia proposta exigiria o conhecimento da função densidade de probabilidade 
(fdp) das variáveis relacionadas que são desconhecidas para os cenários mmWave. Para resolver este problema, são utilizadas as aproximações fdp Gamma. As aproximações analíticas do BER resultaram em diferenças não superiores a $0,5 \mathrm{~dB}$ em relação aos resultados da simulação em alto SNR.

\section{Palavras-Chaves}

Ondas milimétricas; precodificação híbrida; MIMO massivo, sistemas MIMO; MIMO massivo multiusuário 


\section{Table of contents}

1 Introduction $\quad 14$

2 System and channel model $\quad 18$

2.1 System model 18

2.2 Channel model 19

3 Hybrid precoder proposal that maximizes the mutual information $\quad 21$

3.1 Hybrid precoder proposal 21

3.2 Numerical results 23

$4 \quad$ Four different hybrid precoders based on MMSE $\quad 28$

$\begin{array}{lll}4.1 & \text { Hybrid designing approaches } & 29\end{array}$

$\begin{array}{lll}4.1 .1 & \text { [21]-SVD-MMSE algorithm } & 29\end{array}$

4.1.2 [24]-CIA-BD algorithm 30

4.1.3 [29]-CIA-MMSE algorithm 32

4.2 Hybrid precoder/combiner proposal I 32

4.3 Hybrid precoder/combiner proposal II and III 34

4.4 Hybrid precoder/combiner proposal IV 34

4.4.1 Hybrid combiner proposal 34

4.4.2 Hybrid precoder proposal 37

4.5 Data detection approaches 38

4.6 Numerical results 39

5 Method to find fairly tight analytic approximations to the BER performance $\quad 47$

5.1 Semi-analytic approximated BER expression 47

5.2 Analytic approximate BER expression 50

5.3 Hybrid design 51

5.4 Numerical results 52

$6 \quad$ Three stages hybrid precoder proposal $\quad 67$

$\begin{array}{ll}6.1 \text { Hybrid precoder proposal } & 67\end{array}$

$\begin{array}{lll}6.1 .1 & \text { Hybrid design review } & 68\end{array}$

6.1.2 Power allocation matrix proposal 69

$\begin{array}{lll}6.2 & \text { Numerical results } & 71\end{array}$

$\begin{array}{lll}7 & \text { Conclusions } & 76\end{array}$

$\begin{array}{ll}\text { Bibliography } & 79\end{array}$

$\begin{array}{ll}\text { A Appendix } & 84\end{array}$

A.1 General linear constrained MMSE Precoding 84 


\section{List of figures}

Figure 3.1 Representation of the hybrid precoder design idea

Figure 3.2 Mutual information rate achieved by different schemes using $N_{t}=64, N_{r}=4, K=4, N_{s}=2$

Figure 3.3 Mutual information rate achieved by different schemes using $N_{t}=256, N_{r}=4, K=8, N_{s}=2$

Figure 3.4 Mutual information rate achieved by different hybrid precoding schemes as function of the users number with fixed $\mathrm{SNR}=10$ $\mathrm{dB}, N_{t}=256, N_{r}=16, N_{s}=2$, and a variable number of users.

Figure 3.5 BER performance comparison using NMDD. Simulation settings: $N_{t}=64, N_{r}=4, N_{s}=2$, and $K=4$.

Figure 4.1 Design idea of the analog precoder and hybrid combiner

Figure 4.2 The value of $\log _{2}\left(\prod_{i=1}^{n} \lambda_{i}^{2}\right)$, with $\lambda_{1} \geq \lambda_{2} \geq \cdots \geq \lambda_{K N_{s}}$, of different equivalent baseband channels

Figure 4.3 Achievable sum rate using $N_{t}=64, N_{r}=4, N_{s}=2, K=4$ and $K=8$

Figure 4.4 BER results obtained with the MDD and AMDD data detectors and simulation settings $N_{t}=64, N_{r}=4, N_{s}=2$, $K=4$

Figure 4.5 BER results obtained with the NWMDD and NWAMDD data detectors and simulation settings $N_{t}=64, N_{r}=4, N_{s}=2$, $K=4$

Figure 4.6 BER results obtained with the MDD and AMDD data detectors and simulation settings $N_{t}=64, N_{r}=4, N_{s}=2$, $K=8$

Figure 4.7 BER results obtained with the NWMDD and NWAMDD data detectors and simulation settings $N_{t}=64, N_{r}=4, N_{s}=2$, $K=8$

Figure 4.8 BER results obtained with the NWIMDD data detector and simulation settings $N_{t}=64, N_{r}=4, N_{s}=2, K=4$

Figure 4.9 BER results obtained with the NWIMDD data detector and simulation settings $N_{t}=64, N_{r}=4, N_{s}=2, K=8$

Figure 5.1 Simulated and estimated values of the BER performance using P-SVD*-ZF. Common simulation settings $N_{t}=64, N_{s}=2$ and $\hat{\mathbf{B}}=\mathbf{I}_{N_{s}}$

Figure 5.2 Simulated and estimated values of the BER performance using P-SVD*-MMSE*. Simulation settings $N_{t}=64, N_{s}=2$, $K=4, N_{r}=4$, and $\hat{\mathbf{B}}=\mathbf{I}_{N_{s}}$

Figure 5.3 Simulated and estimated values of the BER performance using P-SVD*-MMSE*. Simulation settings $N_{t}=64, N_{s}=2$, $K=8, N_{r}=16$, and $\hat{\mathbf{B}}=\mathbf{I}_{N_{s}}$

Figure 5.4 Simulated and estimated values of the BER performance using P-SVD*-ZF. Simulation settings: MDD, $N_{t}=64, N_{s}=2$, $K=4, N_{r}=4$ and $\hat{\mathbf{B}}_{k}=\mathbf{B}_{k}$. 
Figure 5.5 Simulated and estimated values of the BER performance using P-SVD*-ZF. Simulation settings: NWMDD (NIWMDD), $N_{t}=64, N_{s}=2, K=4, N_{r}=4$ and $\hat{\mathbf{B}}_{k}=\mathbf{B}_{k}$.

Figure 5.6 Simulated and estimated values of the BER performance using P-SVD*-ZF. Simulation settings: MDD, $N_{t}=64, N_{s}=2$, $K=8, N_{r}=16$ and $\hat{\mathbf{B}}_{k}=\mathbf{B}_{k}$.

Figure 5.7 Simulated and estimated values of the BER performance using P-SVD*-ZF. Simulation settings: NWMDD (NIWMDD), $N_{t}=64, N_{s}=2, K=8, N_{r}=16$ and $\hat{\mathbf{B}}_{k}=\mathbf{B}_{k}$.

Figure 5.8 Simulated and estimated values of the BER performance using P-SVD*-MMSE*. Simulation settings: MDD, $N_{t}=64$, $N_{s}=2, K=4, N_{r}=4$ and $\hat{\mathbf{B}}_{k}=\mathbf{B}_{k}$.

Figure 5.9 Simulated and estimated values of the BER performance using P-SVD*-MMSE* ${ }^{*}$, Simulation settings: NWMDD, $N_{t}=64$, $N_{s}=2, K=4, N_{r}=4$ and $\hat{\mathbf{B}}_{k}=\mathbf{B}_{k}$.

Figure 5.10 Simulated and estimated values of the BER performance using P-SVD*-MMSE* ${ }^{*}$. Simulation settings: NIWMDD, $N_{t}=$ 64, $N_{s}=2, K=4, N_{r}=4$ and $\hat{\mathbf{B}}_{k}=\mathbf{B}_{k}$.

Figure 5.11 Simulated and estimated values of the BER performance using P-SVD*-MMSE*,. Simulation settings: MDD, $N_{t}=64$, $N_{s}=2, K=8, N_{r}=16$ and $\hat{\mathbf{B}}_{k}=\mathbf{B}_{k}$.

Figure 5.12 Simulated and estimated values of the BER performance using P-SVD*-MMSE* ${ }^{*}$. Simulation settings: NWMDD, $N_{t}=$ $64, N_{s}=2, K=8, N_{r}=16$ and $\hat{\mathbf{B}}_{k}=\mathbf{B}_{k}$.

Figure 5.13 Simulated and estimated values of the BER performance using P-SVD*-MMSE* ${ }^{*}$. Simulation settings: NIWMDD, $N_{t}=$ $64, N_{s}=2, K=8, N_{r}=16$ and $\hat{\mathbf{B}}_{k}=\mathbf{B}_{k}$.

Figure 5.14 BER performance of P-SVD*-ZF and P-SVD*-MMSE* using NWMDD. Simulation settings: $N_{t}=64, K=4, N_{r}=4, N_{s}=2$, and modulation 16QAM

Figure 6.1 BER performance comparison between our proposals PSVD $^{*}-M_{M S E}^{*}+\mathrm{PA}$ and P-SVD*-MMSE* $N t=64, N_{r}=4$, $N_{s}=2$ and $K=4$

Figure 6.2 BER performance comparison between our proposal PSVD $^{*}-\mathrm{ZF}+\mathrm{PA}$ and P-SVD*-ZF. $N t=64, N_{r}=4, N_{s}=2$ and $K=4$

Figure 6.3 BER performance comparison between our proposals PSVD $^{*}$-MMSE* $+\mathrm{PA}$ and P-SVD*-MMSE* $. N t=64, N_{r}=16$, $N_{s}=2$ and $K=8$

Figure 6.4 BER performance comparison between our proposals $\mathrm{P}$ SVD $^{*}-\mathrm{ZF}+\mathrm{PA}$ and P-SVD ${ }^{*} \mathrm{ZF} . N t=64, N_{r}=16, N_{s}=2$ and $K=8$ 


\section{List of tables}

Table 5.1 Approximated BER accurateness in terms of mean and variance of the $\left|\Delta_{\mathrm{BER}}\right|$ when MDD is used

Table 5.2 Approximated BER accurateness in terms of mean and variance of the $\left|\Delta_{\text {BER }}\right|$ when NWMDD is used

Table 5.3 Approximated BER accurateness in terms of mean and variance of the $\left|\Delta_{\mathrm{BER}}\right|$ when NIMDD is used 59

Table 6.1 Number of times that the water filling algorithm was used 


\section{List of Abreviations}

\begin{tabular}{|c|c|c|}
\hline & $\mathrm{ADC}$ & - Analog to digital converter \\
\hline & $\mathrm{BS}$ & - Base station \\
\hline & $\mathrm{BF}$ & - Beamforming \\
\hline & BER & - Bit error rate \\
\hline & $\mathrm{BD}$ & - Block diagonalization \\
\hline & $\mathrm{DAC}$ & - Digital to analog converter \\
\hline & CIA & - Column iterative algorithm \\
\hline & CSI & - Channel side information \\
\hline & $\mathrm{HB}$ & - Hybrid beamforming \\
\hline & mmWave & - Millimeter-Wave \\
\hline & MMSE & - Minimum mean square error \\
\hline & MS & - Mobile station \\
\hline & MIMO & - $\quad$ Multiple-input and multiple-output \\
\hline & MU-MIMO & - Multi-user MIMO \\
\hline & $\mathrm{RF}$ & - Radio frequency \\
\hline & SNR & - Signal-to-noise ratio \\
\hline & SVD & - Singular value decomposition \\
\hline & $3 \mathrm{D}$ & - Three dimensional \\
\hline & ULA & - Uniform linear array \\
\hline & UPA & - Uniform planar array \\
\hline & WSMSE & - Weighed sum mean square error \\
\hline & $\mathrm{ZF}$ & $-\quad$ Zero forcing \\
\hline
\end{tabular}




\section{1 \\ Introduction}

Millimeter-Wave (mmWave) band communication recently gained more and more attention for the development of short-range high-speed wireless networks. The advantage of this technology lies on its capability to support multi-Gbps throughput [3]. The mmWave frequency bands present an attractive option for designing multi-Gbps wireless links because they offer an abundance of unlicensed or semi-unlicensed bandwidth. The unlicensed $60 \mathrm{GHz}$ band, for example, offers $7 \mathrm{GHz}$ of spectrum spanning 57 to $64 \mathrm{GHz}$ in the United States [2]. However, the higher expected path loss caused by the high frequency carrier, atmospheric gases, water vapor, and atmospheric absorption result in severe link quality degradation [1]. The adverse channel conditions at mmWave frequencies can be compensated with beamforming $(\mathrm{BF})$ algorithms employing a large number of antennas that give a large array gain. An interesting feature of mmWave is that large antenna arrays can occupy a very small area thanks to its small wavelength [4].

Due to their simplicity, systems using uniform linear array (ULA) have been widely studied for use at mmWave frequencies [5-7]. However, uniform planar array (UPA) is capable of using additional vertical degrees of freedom allowing the three dimensional (3D) BF that takes advantage of both elevation and azimuth domain BF to efficiently mitigate interuser interference and eventually increase system capacity.

The conventional fully digital precoders requere one dedicated radio frequency $(\mathrm{RF})$ chain for each antenna element, where an RF chain includes a low-noise amplifier, a down-converter, a digital to analog converter (DAC), an analog to digital converter (ADC) and so on. Then, when the number of antennas is very large, the high cost and power consumptions of mixed signal components implie that the dedication of a separate RF chain for each antenna is highly inefficient. In addition, the number of RF chains is only lower-limited by the number of data streams that are to be transmitted; while the BF gain and diversity order are given by the number of antenna elements if a suitable $\mathrm{BF}$ is made. For these reasons, BF design with limited number of RF chains has recently received significant attention [8].

Two relevant solutions have been proposed to mmWave beamforming. 
The first one is to perform only changes of phases on the signal, i.e., analog beamforming. This technique consists in processing the signal on the RF domain by using low cost phase shifters, however, the users usually receive only a single stream due to his inherent limited capability in managing interference, and therefore a single RF chain is required in each mobile station. The second solution enables spatial multiplexing transmission by using a reduced number of RF chains such that the beamforming is divided between the analog and digital domain, that is known as hybrid beamforming (HB).

A large number of HB designs have been proposed with different approaches. Mainly, there are two HB structures: (i) full-complexity, where each $\mathrm{RF}$ chain is connected to all antenna elements; and (ii) reduced-complexity, where each RF chain is connected to a subset of antenna elements, the complexity reduction is done at the price of performance loss. We particularly select the first one in order to obtain more degrees of freedom. The HB approaches can be also categorized in relation to its channel side information (CSI) requirement: (i) fixed approaches, where a set of beams in many directions are generated previously with no CSI. The literature defines this set as beam codebook, and in every transmission a beam is selected such that the stronger power processed signal is obtained; and (ii) adaptive approaches, that are able to deliver better performances when compared to the fixed approaches requering, however, CSI or partial CSI in each transmission [7].

We addressed our present research to downlink in mmWave multiuser multiple-input and multiple-output (MU-MIMO)systems using adaptive hybrid processing with fully connected structures. The system and channel model for that scenario is described in Chapter 2. Then, Chapter 3 presents our first hybrid precoder proposal, which looks for the maximization of the mutual information upper bound. This problem has already been studied in mmWave systems for the last years, e.g., [1,21,23,24,29,32]. Many proposed solutions try to decrease the distance between the hybrid precoder and a digital one but, this methodology generally leads to iterative complex algorithms. However, the considered problem in this chapter has been widely studied for traditional MIMO system, thus, our research was focused on the adaptation of a solution to mmWave systems. Particularly, Chapter 3 presents the adaptation of [30] to mmWave MU-MIMO scenarios. Numerical results in terms of mutual information rate show the improvement obtained by our proposal in relation to other considered hybrid precoders. However, the results in terms of bit error rate (BER) performance achieved by this proposal is not good due to the maximization of the mutual information could lead to solutions that also increase the inter user interference, and unfortunately, the hybrid precoder resulted 
from our approach has low interference management capability. Thus, in order to decrease the BER, structures based on the classic linear precoders, i.e., MMSE, ZF, are explored in the following chapters.

Chapter 4 presents a different approach to design the hybrid combiner/precoder. The HB design consist of two stages, the first stage performs the analog part of the beamforming that maximizes the effective channel gain, and the second stage applies a low-dimensional baseband precoder to manage the inter user/symbol interference. The system baseband model shows that the signal processed by the mobile station can be interpreted as a received signal in the presence of colored Gaussian noise, therefore, since the digital part of the combiner and precoder do not have constraints for their generation, their designs can be based on any traditional signal processing that takes into account this kind of noise. To the best out knowledge, this was not considered by previous works. A more realistic and appropriate design is described in this chapter. Also, the approaches adopted in the literature for the designing of the combiner'/precoder' analog parts do not try to avoid or even reduce the inter user/symbol interference, they are concentrated on increasing the SNR. We propose a simple solution that decreases the interference while maintaining large SNR. Chapter 4 presents four hybrid precoder/combiner designs, where one of the proposed hybrid combiners reaches the maximum value of the considered cost function according to the Hadamard's inequality. Numerical results illustrate the BER performance improvements resulting from our proposals. In addition, a simple detection approach can be used for data estimation without significant performance loss.

To the best of our knowledge, there are no works presenting exact closed forms expressions for the BER performance in mmWave system. Chapter 5 proposes a method to find fairly tight analytic approximations to the BER performance using different data detection approaches. According to the numerical results, the analytic BER approximations resulted in differences no larger than $0.5 \mathrm{~dB}$ with respect to the simulation results in high SNR. In addition, a variation of our proposed P-SVD*-MMSE* described in Section 4.4 is included.

Chapter 6 proposes a new approach to design the hybrid precoder. This proposal adds a third stage to our previous hybrid precoders proposed in Chapters 4 and 5. This new stage allocates the available transmit power in order to decrease the BER. Numerical results in terms of BER performance show that this new approach can reach an improvement about $0.5 \mathrm{~dB}$ in large SNR values or even better in larger SNR. In Chapter 7, some conclusions are underlined; and finally Appendix A presents some derivations and our 
published papers in this area.

The following notation is used throughout the paper: $\mathbb{C}$ denotes the field of complex numbers; $\mathcal{A}$ is a set; $\mathbf{A}$ is a matrix; $\mathbf{a}$ is a vector; $a$ is a scalar; $\mathbf{A}_{a, b}$, $\mathbf{A}_{a,:}, \mathbf{A}_{:, b}$, denote the $(a, b)$-th entry, $a$-th row, and $b$-th column of the matrix $\mathbf{A}$, respectively; $\mathbf{1}_{a, b}$ is the $a \mathrm{x} b$ all ones matrix; $\mathbf{I}_{N}$ is the $N \mathrm{x} N$ identity matrix; $\operatorname{tr}\{\mathbf{A}\}$ returns the trace of matrix $\mathbf{A} ;\|.\|_{p}$ is the $p$-norm, for the euclidean norm case, $p=2$, the under-index is avoided; $\operatorname{det}($.$) represents the determinant$ function; $\mid$ A $\mid$ returns the product of the nonzero eigenvalues of the square matrix $\mathbf{A} ; \otimes$ is the Kronecker product; $(.)^{T}$ and $(.)^{H}$ denote the transpose and conjugate transpose, respectively; $\mathbb{E}[$.$] is the expectation operator; \mathcal{C N}\left(m, \sigma^{2}\right)$ denotes a complex Gaussian random variable with mean $m$ and variance $\sigma^{2}$; the function $\mathbf{\Psi}(\mathbf{A})$ returns the entries of the matrix $\mathbf{A} \in \mathbb{C}^{n \times m}$ normalized to magnitude 1, i.e., $(\mathbf{\Psi}(\mathbf{A}))_{i, j}=\frac{\mathbf{A}_{i, j}}{\left\|\mathbf{A}_{i, j}\right\|}, i=1, \ldots, n, j=1, \ldots . m ; \operatorname{tr}\{\mathbf{A}\}$ returns the trace of the matrix $\mathbf{A} ;\left[\mathbf{U}, \boldsymbol{\Lambda}, \mathbf{V}^{H}\right]=\operatorname{svd}(\mathbf{A})$ performs a singular value decomposition of matrix $\mathbf{A}$, such that $\mathbf{A}=\mathbf{U} \boldsymbol{\Lambda} \mathbf{V}^{H} ; \mathbf{A}=\mathfrak{R}\{\mathbf{B}\}$ returns the real part of each element in array $\mathbf{B} ; \mathbf{B}=\operatorname{blkdiag}\left\{\mathbf{A}_{1}, \ldots, \mathbf{A}_{N}\right\}$ returns the block diagonal matrix created by aligning the input matrices $\mathbf{A}_{1}, \ldots, \mathbf{A}_{N}$ along the diagonal of $\mathbf{B}$; and $(x)_{+}=\max \{0, x\}$. 


\section{2}

\section{System and channel model}

This chapter describes the system and channel model commonly used by the literature in the mmWave topic through Sections 2.1 and 2.2, respectively.

\section{1}

\section{System model}

We consider downlink mmWave MU-MIMO systems using HB in the base station (BS) and in each mobile station (MS). To enable precoding, we assume that the BS has full channel side information knowledge. In fact, thanks to the sparse nature of mmWave channel, the channel information can be effectively estimated by compressive sensing algorithms [46-48] HB in the BS can be represented by the product between the RF beamformer, $\mathbf{F}_{R F} \in \mathbb{C}^{N_{t} \times N_{R F_{t}}}$, and the baseband beamformer, $\mathbf{F}_{B B} \in \mathbb{C}^{N_{R F_{t}} \times K N_{s}}$. There are $K$ users equipped with $N_{r}$ antennas and $N_{R F_{r}}$ RF chains to process $N_{s}$ streams. The BS has $N_{t}$ antennas and sends $K N_{s}$ streams simultaneously using $N_{R F_{t}}$ RF chains, where $N_{R F_{t}}$ satisfies $K N_{s} \leq N_{R F_{t}} \leq N_{t}$. If $N_{R F_{t}}$ is equal to $N_{t}$, the BS performs digital beamformer [16].

Power normalization is satisfied such that $\left\|\mathbf{F}_{R F} \mathbf{F}_{B B}\right\|_{F}^{2}=K N_{s}$. Then the received signal by the user $k, \mathbf{r}_{k} \in \mathbb{C}^{N_{r} \times 1}$, is expressed as

$$
\mathbf{r}_{k}=\mathbf{H}_{k} \mathbf{F}_{R F} \mathbf{F}_{B B} \mathbf{S}+\mathbf{n}_{k}
$$

where $\mathbf{H}_{k} \in \mathbb{C}^{N_{r} \times N_{t}}$ denotes the channel matrix from the BS to the user $k$ satisfying $\mathbb{E}\left[\left\|\mathbf{H}_{k}\right\|_{F}^{2}\right]=N_{t} N_{r} ; \mathbf{n}_{k} \in \mathbb{C}^{N r \times 1}$ is a complex Gaussian noise vector with zero-mean and covariance matrix $\sigma_{n}^{2} \mathbf{I}_{N_{r}}$, i.e., $\mathcal{C N}\left(0, \sigma_{n}^{2} \mathbf{I}_{N_{r}}\right)$; $\mathbf{s} \in \mathbb{Q}^{K N_{s} \times 1}$ is the data stream vector expressed as the concatenation of the user's stream vectors such that $\mathbf{s}=\left[\mathbf{s}_{1}^{T}, \mathbf{s}_{2}^{T}, \ldots, \mathbf{s}_{K}^{T}\right]^{T}$ with $\mathbb{E}\left[\mathbf{s s}^{H}\right]=\mathbf{I}_{K N_{s}}$ and whose entries belong to a constellation $\mathbb{Q}$. The analog part of the precoder, $\mathbf{F}_{R F}$, is implemented by phase shifters, satisfying $\left\|\left(\mathbf{F}_{R F}\right)_{i, j}\right\|=\frac{1}{\sqrt{N_{t}}}$.

The receiver uses its $N_{R F_{r}}$ RF chains and analog phase shifters to obtain the processed received signal

$$
\mathbf{y}_{k}=\mathbf{W}_{B B_{k}}^{H} \mathbf{W}_{R F_{k}}^{H} \mathbf{H}_{k} \mathbf{F}_{R F} \mathbf{F}_{B B} \mathbf{s}+\mathbf{W}_{B B_{k}}^{H} \mathbf{W}_{R F_{k}}^{H} \mathbf{n}_{k}
$$

where $\mathbf{W}_{R F_{k}} \in \mathbb{C}^{N_{r} \times N_{R F_{r}}}$ is the RF combining matrix and $\mathbf{W}_{B B_{k}} \in \mathbb{C}^{N_{R F_{r}} \times N_{s}}$ denotes the baseband combining matrix of the user $k$. Similarly to the 
$\mathrm{RF}$ precoder, $\mathbf{W}_{R F_{k}}$ is implemented using phase shifters and therefore $\|$ $\left(\mathbf{W}_{R F_{k}}\right)_{i, j} \|=\frac{1}{\sqrt{N_{r}}}[1]$.

Equation (2-2) can be rewritten in baseband terms as follows

$$
\mathbf{y}_{k}=\mathbf{W}_{B B_{k}}^{H} \mathbf{H}_{B B_{k}} \mathbf{F}_{B B} \mathbf{s}+\mathbf{W}_{B B_{k}}^{H} \mathbf{n}_{B B_{k}}
$$

where $\mathbf{H}_{B B_{k}}=\mathbf{W}_{R F_{k}}^{H} \mathbf{H}_{k} \mathbf{F}_{R F}$ represents the equivalent baseband channel of the user $k$ and in a similar way $\mathbf{n}_{B B_{k}} \sim \mathcal{C N}\left(\mathbf{0}, \mathbf{K}_{B B_{k}}\right)$ is the equivalent baseband noise vector, with covariance matrix $\mathbf{K}_{B B_{k}}=\sigma_{n}^{2} \mathbf{W}_{R F_{k}}^{H} \mathbf{W}_{R F_{k}}$. Since the combiner and precoder matrix, $\mathbf{W}_{B B_{k}}$ and $\mathbf{F}_{B B}$, do not have constraints for its generation, they can be designed from signal processing approaches that take into account the colored Gaussian noise. Therefore, the problem is in the selection of the analog matrix $\mathbf{W}_{R F_{k}}$ and $\mathbf{F}_{R F}$, such that an equivalent baseband channel that facilitates the digital processing is obtained.

The signal-to-noise ratio (SNR) is defined as

$$
\begin{aligned}
\mathrm{SNR} & =\frac{\mathbb{E}\left[\left\|\mathbf{F}_{R F} \mathbf{F}_{B B} \mathbf{s}\right\|^{2}\right]}{\sigma_{n}^{2}} \\
& =\frac{\left.\operatorname{Tr}\left(\mathbf{F}_{R F} \mathbf{F}_{B B} \mathbb{E}\left[\mathbf{s s}^{H}\right] \mathbf{F}_{B B}^{H} \mathbf{F}_{R F}^{H}\right]\right)}{\sigma_{n}^{2}} \\
& =\frac{\left\|\mathbf{F}_{R F} \mathbf{F}_{B B}\right\|_{F}^{2}}{\sigma_{n}^{2}}=\frac{K N_{s}}{\sigma_{n}^{2}}=\frac{E_{T}}{\sigma_{n}^{2}}
\end{aligned}
$$

where $E_{T}=K N_{s}$ represents the total energy available at the BS for transmission.

\section{2}

\section{Channel model}

There are typically two models widely used in the mmWave literature for indoor scenarios, the Saleh-Valenzuela channel model and the extended Saleh-Valenzuela geometric model. The first one considers the resulting channel as a sum of two clustered multi-path, a cluster with line-of-sight (LOS) paths and other with non-line-of-sight (NLOS) paths [17,41]. The second one captures more accurately the mathematical structure by dropping off the cluster with NLOS due to the limited scattering at high frequency and the antenna correlation in tightly packed arrays [1, 16, 42-44].

Thus, we considered the mmWave channel as follows [44]

$$
\mathbf{H}_{k}=\sqrt{\frac{N_{t} N_{r}}{N_{p}}} \sum_{p=1}^{N_{p}} \alpha_{k, p} \mathbf{d}_{N_{r}}\left(\phi_{k, p}^{r}, \theta_{k, p}^{r}\right) \mathbf{d}_{N_{t}}\left(\phi_{k, p}^{t}, \theta_{k, p}^{t}\right)^{H}
$$


where $N_{p}$ is the number of multi-path components in the channel; $\alpha_{k, p} \backsim$ $\mathcal{C N}(0,1)$ is the complex gain of the $p$-th multi-path component in the channel for the $k$-th user, whereas $\phi_{k, p}^{r}\left(\theta_{k, p}^{r}\right)$ and $\phi_{k, p}^{t}\left(\theta_{k, p}^{t}\right)$ are its azimuth (elevation) angles of arrival and departure, respectively [1]. We consider the use of an uniform planar array (UPA) formed by $N_{t}=N_{t_{h}} N_{t_{v}}\left(N_{r}=N_{r_{h}} N_{r_{v}}\right)$ antennas, $N_{t_{h}}\left(N_{r_{h}}\right)$ antennas in the horizontal side and $N_{t_{v}}\left(N_{r_{v}}\right)$ antennas in the vertical side, with the antenna spacing of half wave length at the transmitter (receiver), whose response is given by:

$$
\mathbf{d}_{N_{t}}(\phi, \theta)=\mathbf{d}_{N_{t_{h}}}(\pi \cos (\phi) \sin (\theta)) \otimes \mathbf{d}_{N_{t_{v}}}(\pi \cos (\theta))
$$

with

$$
\mathbf{d}_{M}(\psi)=\frac{1}{\sqrt{M}}\left[1, e^{j \psi}, \ldots, e^{j(M-1) \psi}\right]^{T} \in \mathbb{C}^{M \times 1}
$$




\section{Hybrid precoder proposal that maximizes the mutual infor- mation}

This chapter presents a new approach to design an hybrid precoder through the maximization of the mutual information upper bound considering mmWave MU-MIMO system. This problem has been widely studied on traditional MIMO systems. The work in [30] presents a digital precoder design for single user MIMO. Our proposal performs the adaptation of [30] to MU-MIMO mmWave environments. Numerical results in terms of mutual information rate show the improvement obtained by our proposal in relation to the other considered hybrid precoders.

The remainder of this chapter is organized as follows: Section 3.1 describes our proposal of the hybrid precoder design, and in Section 3.2 some simulations results are shown.

\section{1}

\section{Hybrid precoder proposal}

In order to focus our attention only on the hybrid precoder design, we considered that $\mathbf{W}_{B B_{k}}^{H} \mathbf{W}_{R F_{k}}^{H} \mathbf{W}_{R F_{k}} \mathbf{W}_{B B_{k}} \approx \mathbf{I}_{N_{s}}$, thus the upper bound of the mutual information rate for downlink mmWave MU-MIMO system is defined as follows

$$
\mathbf{I}=\log _{2} \operatorname{det}\left(\mathbf{I}_{K N s}+\frac{1}{\sigma_{n}^{2}} \mathbf{F}_{B B}^{H} \mathbf{F}_{R F}^{H} \mathbf{H}^{H} \mathbf{H} \mathbf{F}_{R F} \mathbf{F}_{B B}\right)
$$

where $\mathbf{H}=\left[\begin{array}{llll}\mathbf{H}_{1}^{T} & \mathbf{H}_{2}^{T} & \cdots & \mathbf{H}_{K}^{T}\end{array}\right]^{T}$ is the stacking of all users' channel matrices. To design the hybrid precoder, it is desirable to find a couple of matrix, $\mathbf{F}_{B B}$ and $\mathbf{F}_{R F}$, such that maximizes this upper bound under the hardware and power constraints considered in mmWave scenarios. Thus, we consider the problem

$$
\begin{aligned}
& \max _{\mathbf{F}_{R F}, \mathbf{F}_{B B}} \log _{2} \operatorname{det}\left(\mathbf{I}_{K N s}+\frac{1}{\sigma_{n}^{2}} \mathbf{F}_{B B}^{H} \mathbf{F}_{R F}^{H} \mathbf{H}^{H} \mathbf{H F}_{R F} \mathbf{F}_{B B}\right) \\
& \text { s.t. }\left\|\mathbf{F}_{R F} \mathbf{F}_{B B}\right\|_{F}^{2}=K N s,\left\|\left(\mathbf{F}_{R F}\right)_{i, j}\right\|=1 / \sqrt{N_{t}} \forall i, j
\end{aligned}
$$

which has already studied before e.g., [1, 21, 23, 24, 29, 32]. Our approach to 
solve (3-2) is based on [30], whose authors describe a methodology to find a digital precoder for a single user MIMO scenario.

In order to continue with the exposition of our proposal, let us introduce the following singular value decomposition (SVD):

$$
\left[\mathbf{V}_{k}, \boldsymbol{\Lambda}_{k}, \mathbf{V}_{k}^{H}\right]=\operatorname{svd}\left(\mathbf{H}_{k}^{H} \mathbf{H}_{k}\right)
$$

Using the analog part of the precoder is possible address the beamforming to the $N_{s}$ principal eigenvectors of $\mathbf{H}_{k}^{H} \mathbf{H}_{k}$ and stack them in a matrix, i.e., $\mathbf{F}_{R F}=\left[\begin{array}{lll}\mathbf{F}_{R F_{1}} & \ldots & \mathbf{F}_{R F_{K}}\end{array}\right]$ where $\mathbf{F}_{R F_{k}}=\boldsymbol{\Psi}\left(\left(\mathbf{V}_{1}\right)_{:, 1: N_{s}}\right)$ represents the submatrix of $\mathbf{F}_{R F}$ that corresponds to the analog precoder part of the user $k$. Note that $\boldsymbol{\Psi}\left(\left(\mathbf{V}_{k}\right)_{:, 1: N_{s}}\right)$ is a good approximation of $\left(\mathbf{V}_{k}\right)_{:, 1: N_{s}}$ when only phase shifters are used. Then, the problem in (3-2) can be rewritten in terms of the equivalent baseband channel, $\tilde{\mathbf{H}}=\mathbf{H F}_{R F}$, as follows

$$
\begin{array}{r}
\max _{\mathbf{F}_{B B}} \log _{2} \operatorname{det}\left(\mathbf{I}_{K N s}+\frac{1}{\sigma_{n}^{2}} \mathbf{F}_{B B}^{H} \tilde{\mathbf{H}}^{H} \tilde{\mathbf{H}} \mathbf{F}_{B B}\right) \\
\text { s.t. }\left\|\mathbf{F}_{R F} \mathbf{F}_{B B}\right\|_{F}^{2}=K N s
\end{array}
$$

Since no analog constraits are considered, it can be applied a digital extention of the methodology described in [30] to a multi-user scenario. Thus, the following SVD is considered

$$
\left[\tilde{\mathbf{V}}_{k}, \tilde{\boldsymbol{\Lambda}}_{k}, \tilde{\mathbf{V}}_{k}^{H}\right]=\operatorname{svd}\left(\frac{1}{\sigma_{n}^{2}} \tilde{\mathbf{H}}_{k}^{H} \tilde{\mathbf{H}}_{k}\right)
$$

where $\tilde{\mathbf{H}}_{k}=\mathbf{H}_{k} \mathbf{F}_{R F}$. Thus, the submatrix of $\mathbf{F}_{B B}$ that corresponds to the digital precoder part of the user $k, \mathbf{F}_{B B_{k}}$, can take the first $N_{s}$ columns of $\tilde{\mathbf{V}}_{k}$ with no approximations, i.e., $\mathbf{F}_{B B_{k}}=\left(\tilde{\mathbf{V}}_{1}\right)_{:, 1: N_{s}}$. Figure 3.1 illustrates the motivation behind above operations. Our proposal to solve (3-4) is approached by the maximization described in Figure 3.1. Observe that the proposed methodology produces that the resulting product is diagonal matrix equal to $\tilde{\Lambda}_{k}$, which maximize the considered determinat and aims indirectly to the maximization of (3-4).

In order to improve the diagonal values of the resulting matrix inside of the determinat in (3-4), the digital part of the precoder is constructed as

$$
\mathbf{F}_{B B}=\left[\begin{array}{lll}
\left(\tilde{\mathbf{V}}_{1}\right)_{:, 1: N_{s}} & \cdots & \left(\tilde{\mathbf{V}}_{K}\right)_{:, 1: N_{s}}
\end{array}\right] \boldsymbol{\Phi}
$$

where $\boldsymbol{\Phi}$ is a diagonal power loading matrix obtained via water-filling using the entries of $\tilde{\Lambda}=\operatorname{blkdiag}\left(\tilde{\Lambda}_{1}, \ldots, \tilde{\Lambda}_{K}\right)$. An option to the implement the waterfilling is presented in Algorithm 1. Algorithm 2 summarizes all steps for the proposed hybrid precoder. 


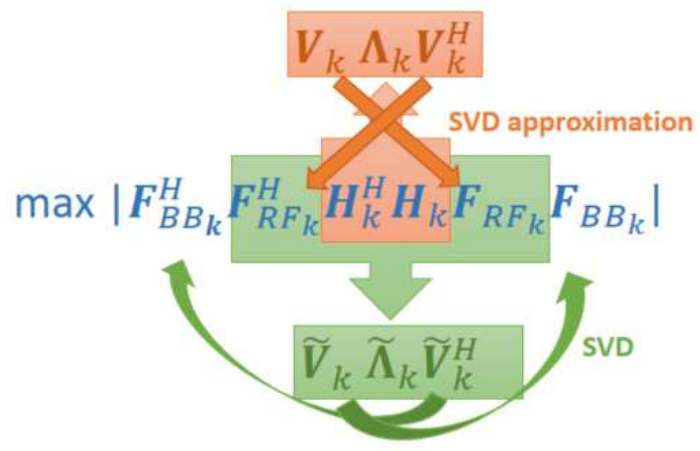

Figure 3.1: Representation of the hybrid precoder design idea

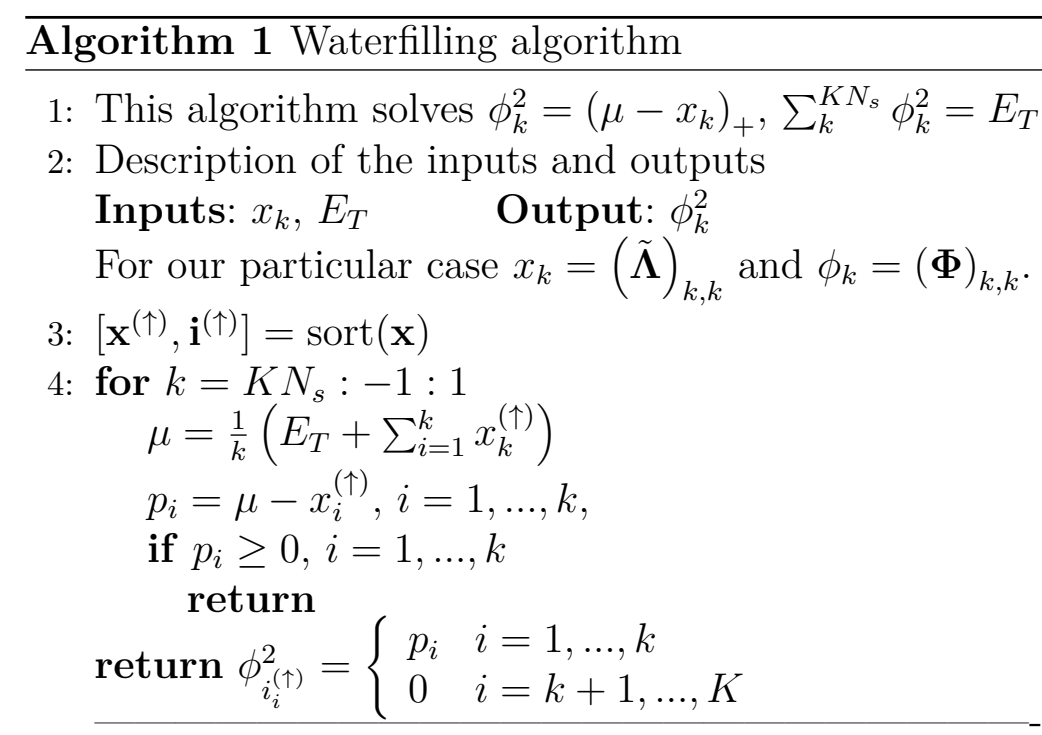

Notation: $a_{k}$ represents the $k$-th component of the vector a.The function $\left[\mathbf{a}^{(\uparrow)}, \mathbf{i}^{(\uparrow)}\right]=\operatorname{sort}(\mathbf{a})$ sorts the elements of $\mathbf{a}$ in ascending order, $\mathbf{a}^{(\uparrow)}$, and stack the ordering index in a vector, $\mathbf{i}^{(\uparrow)}$, such that $a_{k}^{(\uparrow)}=a_{i_{k}^{(\uparrow)}}$.

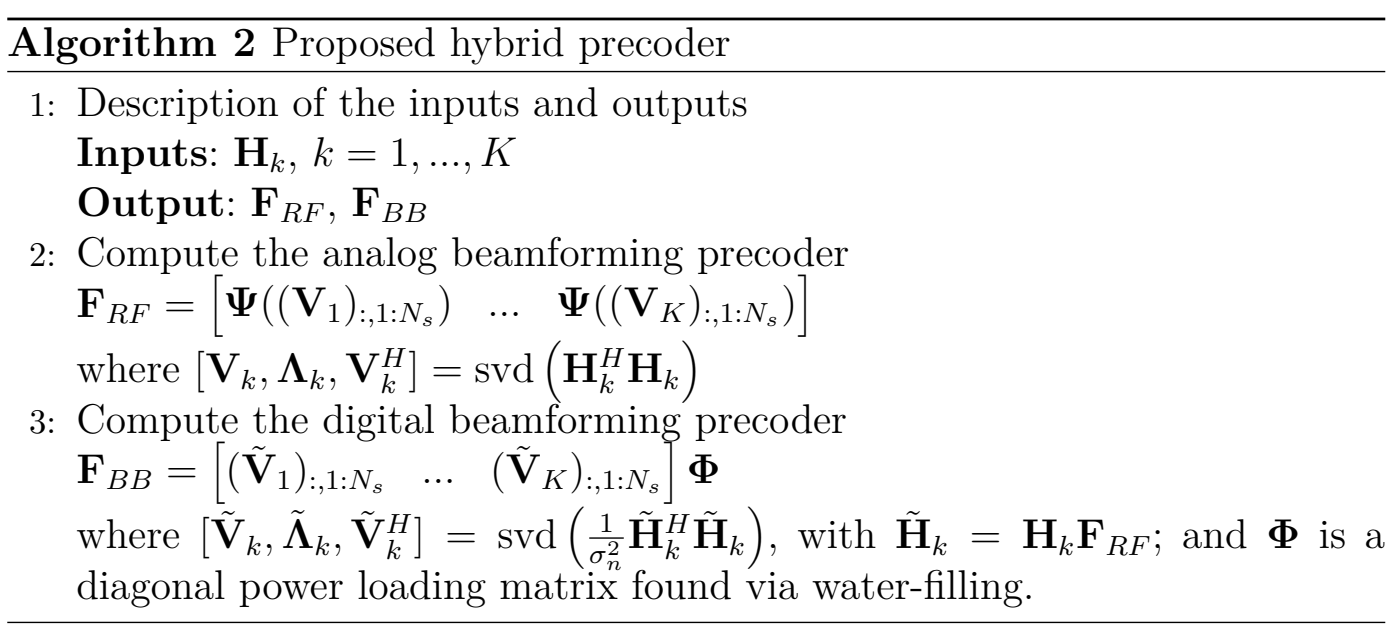

\section{2}

\section{Numerical results}

In the simulations, the users' channels are generated with $N_{p}=10$ multipath components, the azimuth and elevation departure angles values are given 
by a random variable with uniform distribution in the interval of $(0,2 \pi)$ and $(0, \pi)$, respectively. The UPAs have square formats for both transmitter and receivers, i.e., $N_{t_{h}}=N_{t_{v}}={\sqrt{N_{t}}}_{\text {and }} N_{r_{h}}=N_{r_{v}}=\sqrt{N}_{r}$. The minimum allowed setting for RF chains number is used, $N_{R F_{t}}=K N_{s}$ and $N_{R F_{r}}=N_{s}$. The results are averaged over $10^{5}$ channels generations for each user.

The following scenarios are considered: In Figure 3.2 the BS has $N_{t}=64$ antennas and sends $N_{s}=2$ streams to $K=4$ users using $N_{R F_{t}}=8 \mathrm{RF}$ chains. The users are equipped with $N_{r}=4$. In Figure 3.3 the BS has $N_{t}=256$ antennas and sends $N_{s}=2$ streams to $K=8$ users equipped with $N_{r}=4$. In Figure 3.4 the SNR is fixed to $10 \mathrm{~dB}$, the BS has $N_{t}=256$ antennas and sends $N_{s}=2$ streams to different number of users equipped with $N_{r}=16$.

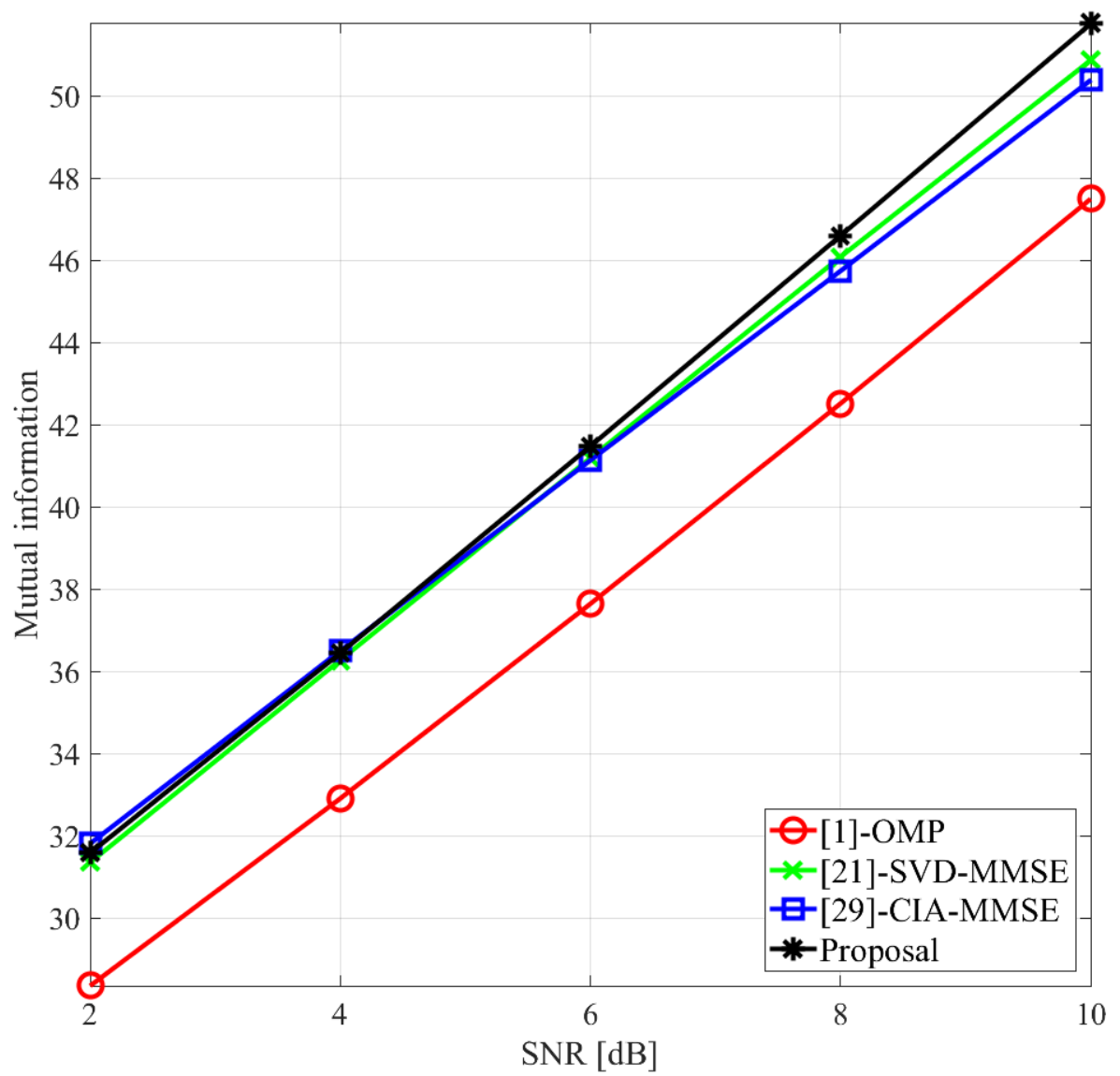

Figure 3.2: Mutual information rate achieved by different schemes using $N_{t}=64, N_{r}=4, K=4, N_{s}=2$

From the above figures, it can be observed that our proposed hybrid precoder overcomes all the other considered hybrid precoders in terms of mutual information rate with a lower complexity due to no matrix inversion procedures are needed. The proposed precoder complexity is about $O\left(N_{t}^{3}\right)$, how- 


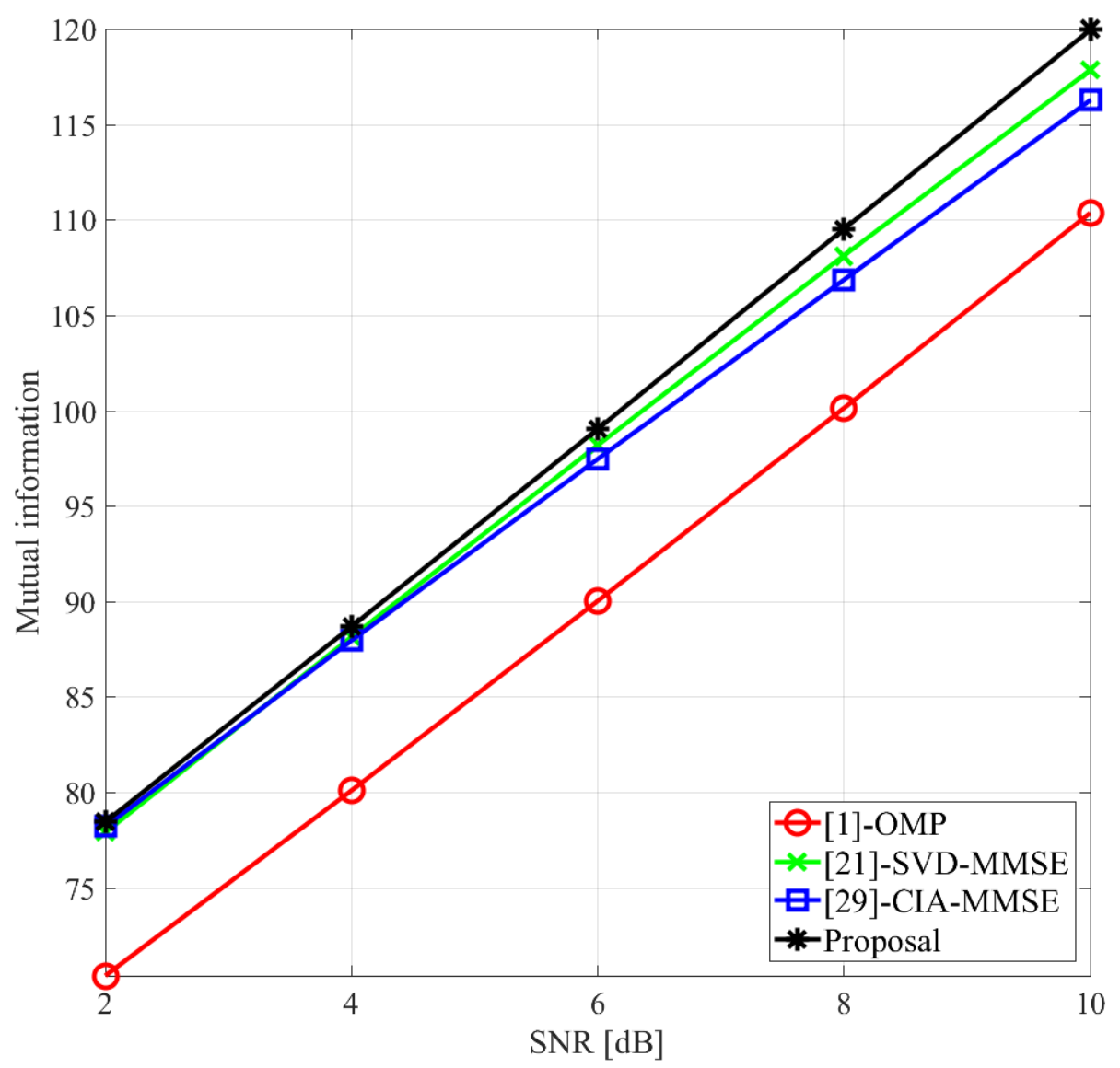

Figure 3.3: Mutual information rate achieved by different schemes using $N_{t}=256, N_{r}=4, K=8, N_{s}=2$

ever, for [1]-OMP, [21]-SVD-MMSE and [29]-CIA-MMSE are $O\left(N_{R F_{t}} N_{t}^{3} N_{r}^{3}\right)$, $O\left(N_{R F_{t}} N_{t}^{3}\right)$, and $O\left(N_{R F_{t}} N_{t}^{3}\right)$, respectively.

It is interesting to know the behavior of our proposal in terms of BER. Therefore, Figure 3.5 presents the BER performance obtained in the scenario considered in Figure 3.2. The considered detection scheme is the noise whitening operation followed by the minimum distance detection (NMDD) (see Section 4.5 for more details). It is worth highlighthing that the hybrid designs described in [21] and [29] propose a hybrid combiner, in contrast in our proposal, for illustrative purposes only, it is considered an all digital zero forcing combiner as follows

$$
\mathbf{W}_{k}=\mathbf{W}_{R F_{k}} \mathbf{W}_{B B_{k}}=\left(\left(\tilde{\mathbf{H}}_{k}^{H} \tilde{\mathbf{H}}_{k}\right)^{-1} \tilde{\mathbf{H}}_{k}^{H}\right)^{H}
$$

In this figure, it can be observed that our hybrid precoder proposal does not achieve a good performance due to its low capacity of interference management. Precoder designs through techniques that maximizes the mutual information could lead to solutions that not only increase the information 


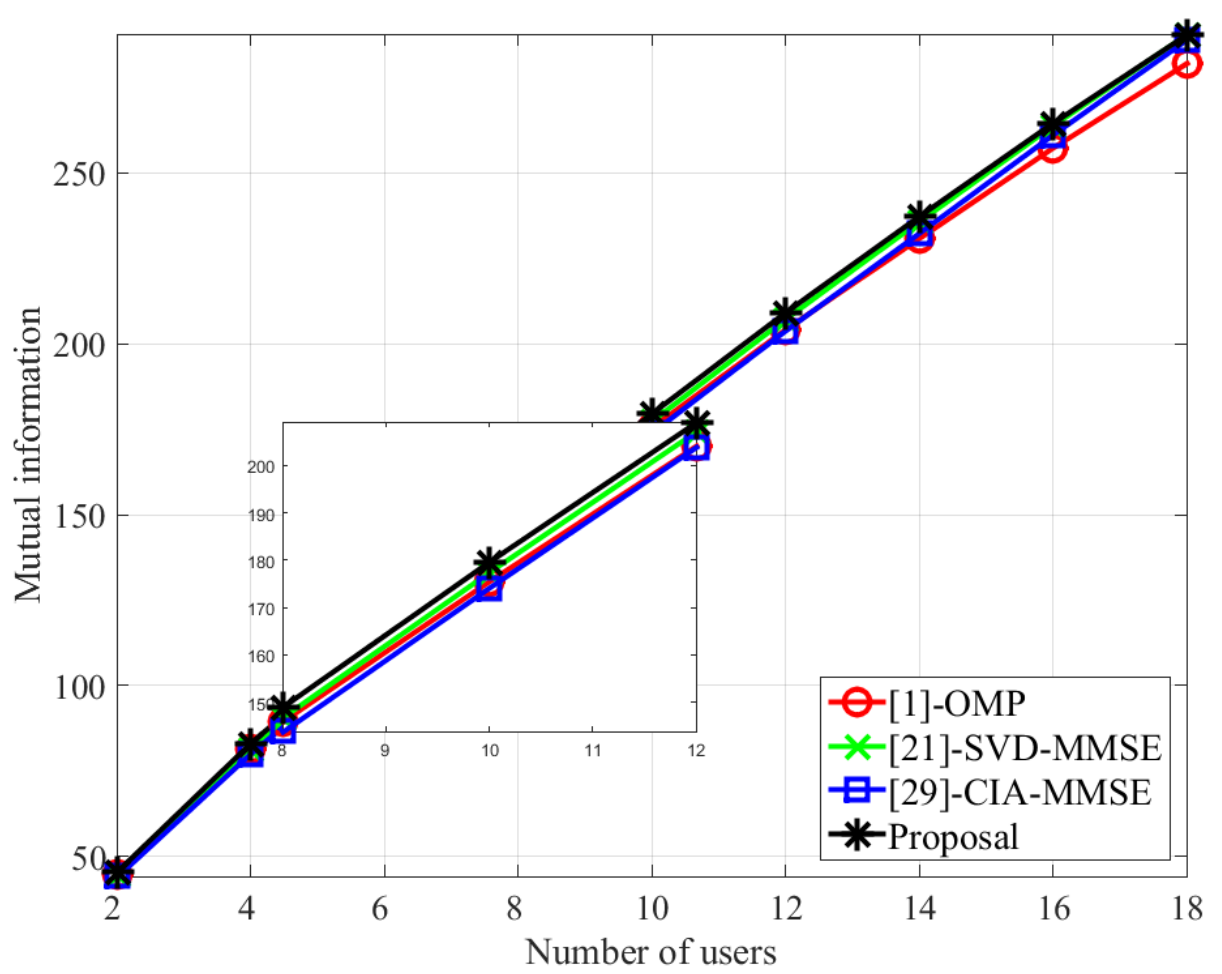

Figure 3.4: Mutual information rate achieved by different hybrid precoding schemes as function of the users number with fixed $\mathrm{SNR}=10 \mathrm{~dB}, N_{t}=256$, $N_{r}=16, N_{s}=2$, and a variable number of users.

between the BS and an user but also increase the inter user interference. In this case, for example, the resulted interference could not be mitigated even using an all digital combiner. Therefore, other precoding strategies must take into account to obtain a better BER performance. The next chapter describes four hybrid precoders/combiners based on the MMSE linear filter precoder. 
Chapter 3. Hybrid precoder proposal that maximizes the mutual information 27

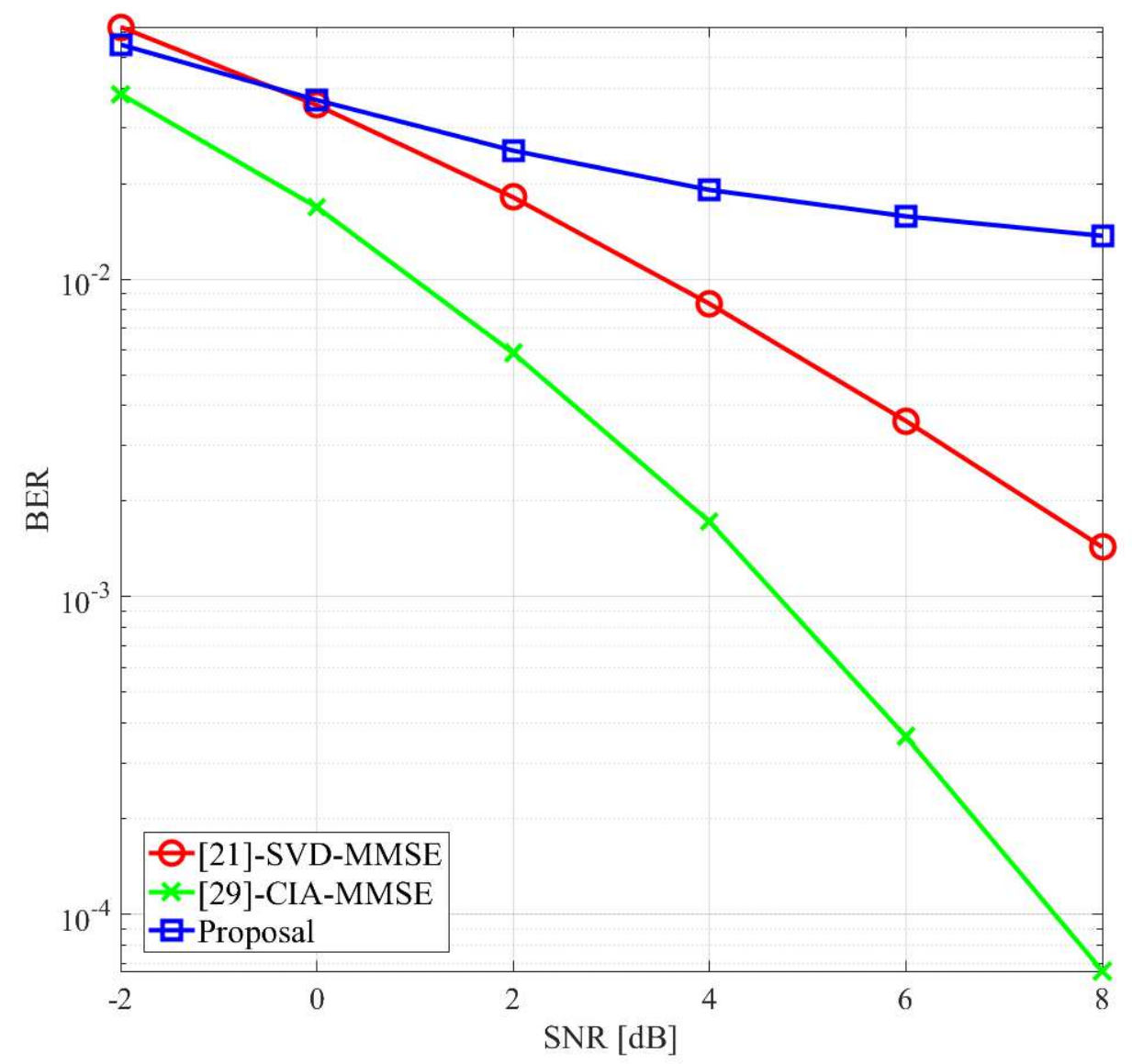

Figure 3.5: BER performance comparison using NMDD. Simulation settings: $N_{t}=64, N_{r}=4, N_{s}=2$, and $K=4$. 


\section{4}

\section{Four different hybrid precoders based on MMSE}

Several approaches have been considered for hybrid processing. The work in [1] is one of the most popular in the literature, the authors exploit the spatial structure of mmWave channels to formulate the precoding/combining problem as a sparse reconstruction problem and they proposed the principle of basis pursuit as tool for its solution. This idea motivated other authors to continue developing hybrid precoders based on sparse reconstruction, e.g., [18-20]. Similar approaches were taken in $[23,33,34]$, where a digital precoder/combiner is defined and then a complex algorithm is proposed to find a hybrid approximation, e.g., gradient descent, considering a weighed sum mean square error (WSMSE) minimization problem or using the orthogonal matching pursuit algorithm. In [35], an algorithm based on manifold optimization is proposed. In each iteration of the algorithm, it assumes a given digital precoder and develops a conjugate gradient method to find an analog precoder that is a local minimizer of the approximation gap from the fully-digital one. Next, the digital precoder is computed using a least squares solution. This method achieves good performance but suffers from high complexity and run time. Nevertheless, more recently the works as $[21,24,29]$ lead to more successful methodologies, which are described briefly in this chapter.

We propose four hybrid combiner/precoder for downlink mmWave massive MU-MIMO systems. The designing of a hybrid combiner/precoder is divided in two parts, analog and digital. The system model in baseband shows that the signal processed by the mobile station can be interpreted as a received signal in the presence of colored Gaussian noise, therefore, since both the digital part of the combiner in the receiver and precoder do not have constraints for their generation, they can be designed using any traditional signal processing approaches that take into account this kind of noise. However, to the best of our knowledge, the previous works do not consider colored noise, e.g., [16, 21,24]. A more realistic and appropriate design is described in this chapter. Two of our proposals consist in the improvement of the digital part of the works in $[21,29]$.

On the other hand, typical approaches for the designing of the analog parts of both the combiner and the precoder are focused on increasing 
the signal-to-noise ratio (SNR) or the signal-to-interference-plus-noise ratio (SINR) but without presenting bit error rate (BER) results, e.g., $[49,50]$. In this chapter we propose two simple solutions that are enable decreasing the inter symbol interference while keeping SNR large. The first proposal consists in the improvement of the iterative algorithm proposed in [24] through a recursive algorithm, and our second proposal is based on singular value decomposition (SVD), which reach excellent performance with much less complexity. In addition one of the the proposed hybrid combiners reaches the maximum value of our objective function according with the Hadamard's inequality. Numerical results in different environments show the improvement obtained by our proposals in relation to the considered hybrid combiner/precoder [21, 24, 29].

The remainder of this chapter is organized as follows: Section 4.1 resumes the hybrid design approaches described in [21,24,29]; Section 4.2, 4.3 and 4.4 are dedicated to describe our proposals. Section 4.5 presents four sub-optimal data detection approaches; and finally, in Section 4.6 simulations results are shown.

\section{1}

\section{Hybrid designing approaches}

This section presents three different approaches for designing the HB for both the transmitter and the receivers. The common factor in these designs is that they use a HB generation divided in two stages, where the first (second) stage obtains the analog (digital) part of the precoder and of the combiner. In order to stress the main characteristic of each stage in a HB design, we considered the following notation to refer to them hereafter: [-]-S $\mathrm{S}_{1}-\mathrm{S}_{2}$, where the first term indicates the reference number and the description of $S_{1}\left(S_{2}\right)$ is related to the first (second) stage-characteristic. An asterisk appearing as an upper index, ${ }^{*}$, in a given characteristic means that it has been modified. In addition, the replacement of the reference number by the letter $\mathrm{P}$ is used to refer to our proposals.

\subsection{1}

\section{[21]-SVD-MMSE algorithm}

The design of the HB in [21] is based on channel knowledge by each user, the analog combiner of each user is independently designed based on the singular value decomposition (SVD), while the analog precoder is obtained by conjugate transposition to maximize the effective channel gain. Then, with the resulting equivalent baseband channel, low dimensional baseband precoders can be efficiently applied, e.g., MMSE or ZF filter. The Algorithm 3 resumes the 
steps for the HB designing in [21]. Note that the considered MMSE filter in the step 4 is a pseudo MMSE linear precoder. A more appropriate expression for the MMSE filter has been demonstrated in [50] and for completeness purpose the deduction applied in our proposals is showed in Appendix A.

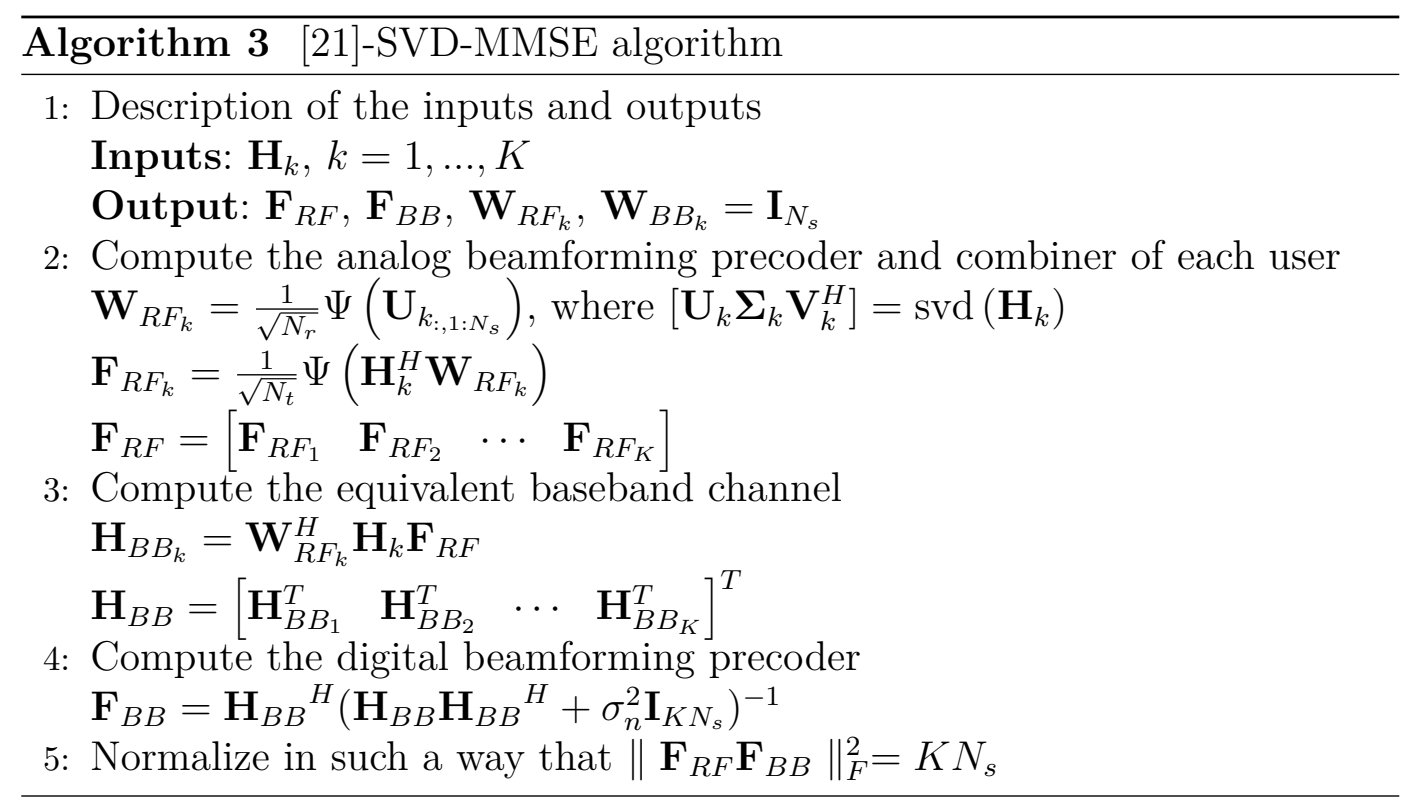

\subsection{2}

\section{[24]-CIA-BD algorithm}

In [24], a methodology to maximize the sum-rate systems can be found, the authors focused on the design of the equivalent baseband channel, i.e., the analog part of the combiner and precoder, and eliminated the interference through baseband block diagonalization (BD) precoding.

The analog part of the combiner is obtained through the optimization problem:

$$
\begin{array}{r}
\quad \max _{R F_{k}} \operatorname{det}\left(\mathbf{W}_{R F_{k}}^{H} \mathbf{A}_{k} \mathbf{W}_{R F_{k}}\right) \\
\text { s.t. }\left\|\left(\mathbf{W}_{R F_{k}}\right)_{i, j}\right\|=1 / \sqrt{N_{r}} \forall i, j
\end{array}
$$

where $\mathbf{A}_{k}=\mathbf{H}_{k} \mathbf{H}_{k}^{H}$. The solution of (4-1) is reached using an column iterative algorithm (CIA) defined in [26] and presented in Algorithm 4 [24].

The analog part of the precoder is obtained using the Algorithm 4 over the objective function in (4-2). 


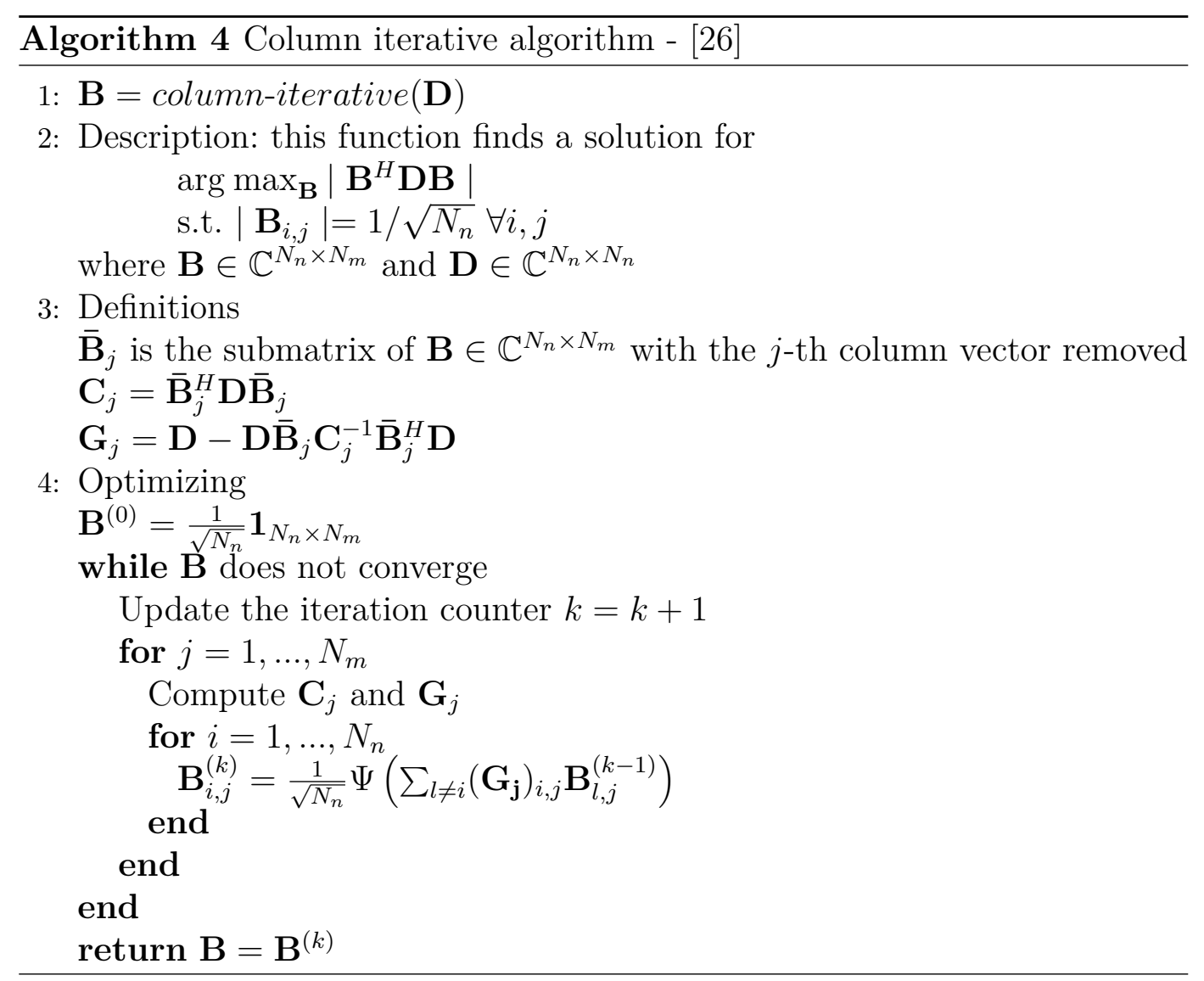

$$
\begin{array}{r}
\max _{\mathbf{F}_{R F}} \operatorname{det}\left(\mathbf{F}_{R F}^{H} \mathbf{A} \mathbf{F}_{R F}\right) \\
\text { s.t. }\left\|\left(\mathbf{F}_{R F}\right)_{i, j}\right\|=1 / \sqrt{N_{t}} \forall i, j
\end{array}
$$

where $\mathbf{A}=\mathbf{H}^{H} \mathbf{W}_{R F} \mathbf{W}_{R F}^{H} \mathbf{H}$, with $\mathbf{W}_{R F}=\operatorname{blkdiag}\left\{\mathbf{W}_{R F_{1}}, \ldots, \mathbf{W}_{R F_{K}}\right\}$ and $\mathbf{H}=\left[\begin{array}{lll}\mathbf{H}_{1}^{T} & \ldots & \mathbf{H}_{K}^{T}\end{array}\right]^{T}$. Algorithm 5 presents a global summary of the analog beamforming design in [24].

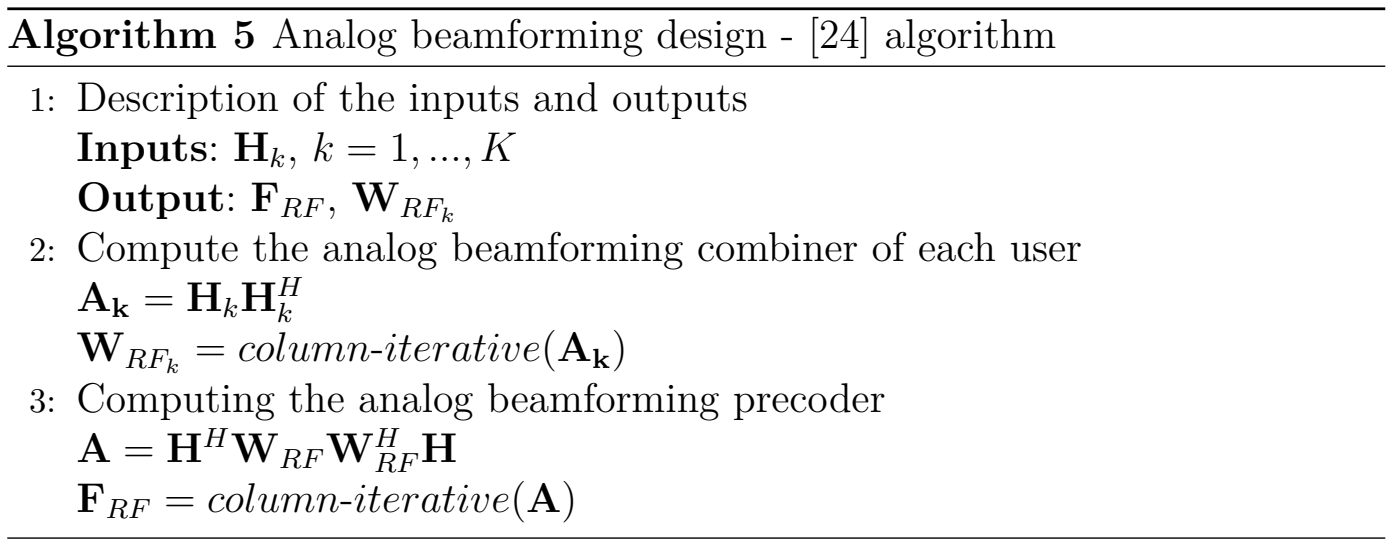

For the digital beamforming part, the $\mathrm{BD}$ is considered. A review of the $\mathrm{BD}$ precoder is presented in Algorithm 6 [25], where the inputs are the 
equivalent baseband channels of the users, $\mathbf{H}_{B B_{k}}$, and outputs are $\mathbf{W}_{B B_{k}}$ and $\mathbf{F}_{B B}$. In addition, a normalization constant has to be computed to satisfy the constraint $\left\|\mathbf{F}_{R F} \mathbf{F}_{B B}\right\|_{F}^{2}=K N_{s}$.

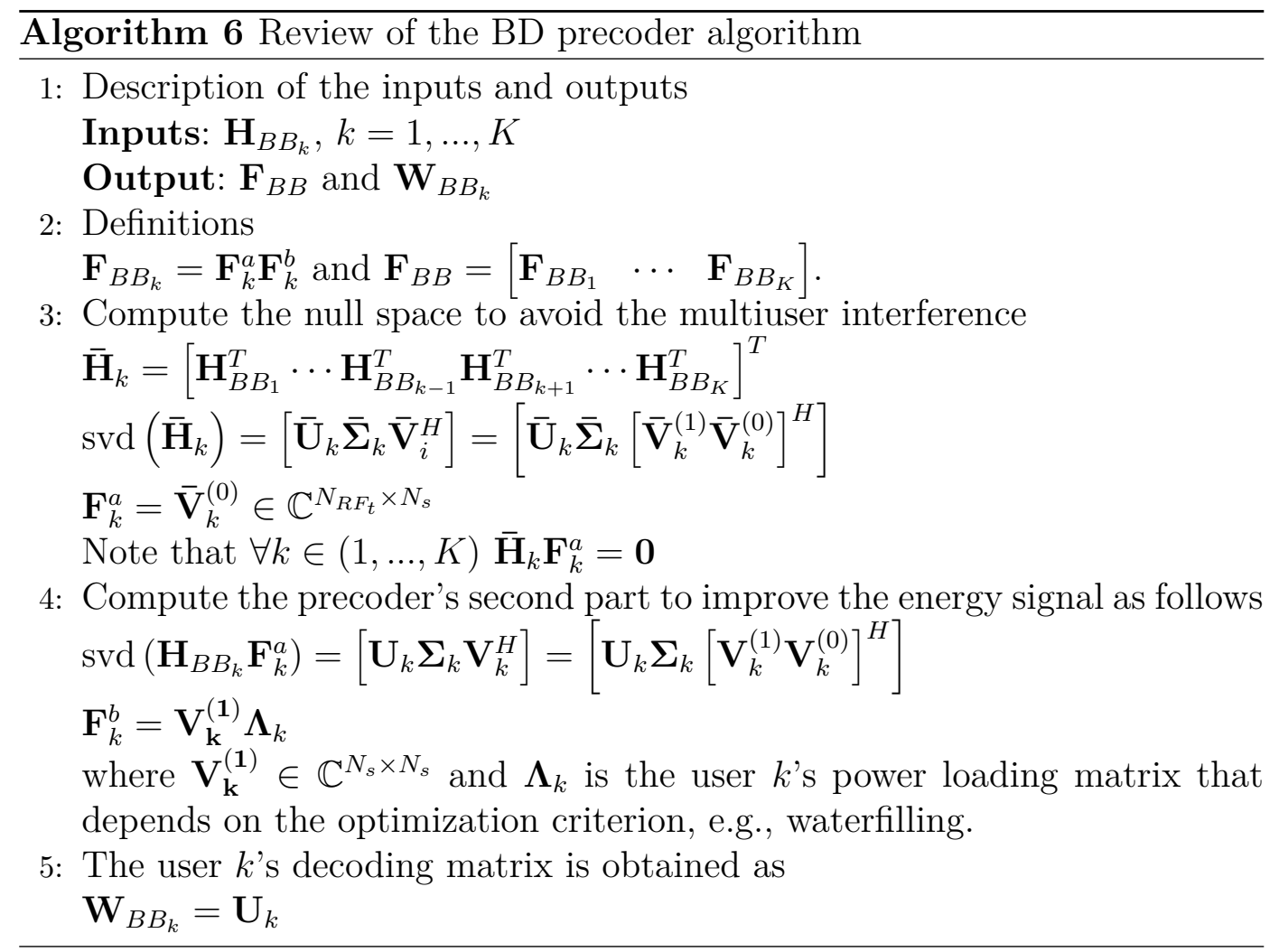

\subsection{3}

\section{[29]-CIA-MMSE algorithm}

Our previous work in [29], [29]-CIA-MMSE, consists in changing the digital beamforming part of [24]-CIA-BD for the pseudo MMSE filter precoder defined in step 4 of the Algorithm 3 instead of a BD filter. In this approach the hybrid combiner complexity decreases because $\mathbf{W}_{B B_{k}}=\mathbf{I}_{N_{s}}$, which means that just analog beamforming is used in the receivers.

\section{2}

\section{Hybrid precoder/combiner proposal I}

Our first proposal, P-CIA*-MMSE*, is described as follows. According to [24] the analog part of the combiner can be designed from the maximization of the determinant of $\mathbf{H}_{B B_{k}} \mathbf{H}_{B B_{k}}^{H}$, where $\mathbf{H}_{B B_{k}}=\mathbf{W}_{R F_{k}}^{H} \mathbf{H}_{k} \mathbf{F}_{R F}$, which means consider the following problem 


$$
\begin{array}{r}
\max _{\mathbf{F}_{R F}, \mathbf{W}_{R F_{k}}} \operatorname{det}\left(\mathbf{W}_{R F_{k}}^{H} \mathbf{H}_{k} \mathbf{F}_{R F} \mathbf{F}_{R F}^{H} \mathbf{H}_{k}^{H} \mathbf{W}_{R F_{k}}\right) \\
\text { s.t. }\left\|\left(\mathbf{F}_{R F}\right)_{i, j}\right\|=1 / \sqrt{N_{t}},\left\|\left(\mathbf{W}_{R F_{k}}\right)_{l, m}\right\|=1 / \sqrt{N_{r}}
\end{array}
$$

Considering an ideal case with no multiuser interference, (4-3) can be simplified to

$$
\begin{aligned}
\max _{\mathbf{F}_{R F_{k}}, \mathbf{W}_{R F_{k}}} \operatorname{det}\left(\mathbf{W}_{R F_{k}}^{H} \mathbf{H}_{k} \mathbf{F}_{R F_{k}} \mathbf{F}_{R F_{k}}^{H} \mathbf{H}_{k}^{H} \mathbf{W}_{R F_{k}}\right) \\
\text { s.t. }\left\|\left(\mathbf{F}_{R F_{k}}\right)_{i, j}\right\|=1 / \sqrt{N_{t}},\left\|\left(\mathbf{W}_{R F_{k}}\right)_{l, m}\right\|=1 / \sqrt{N_{r}}
\end{aligned}
$$

where $\mathbf{F}_{R F_{k}}$ is the submatrix of $\mathbf{F}_{R F}$ corresponding to the analog precoder of the user $k$. To solve this non-convex optimization problem, we can use the column iterative algorithm used in [24] (see Algorithm 4) and to alleviate the dependence between $\mathbf{W}_{R F_{k}}$ and $\mathbf{F}_{R F_{k}}$, a recursive algorithm is considered as illustrated in Algorithm 7.

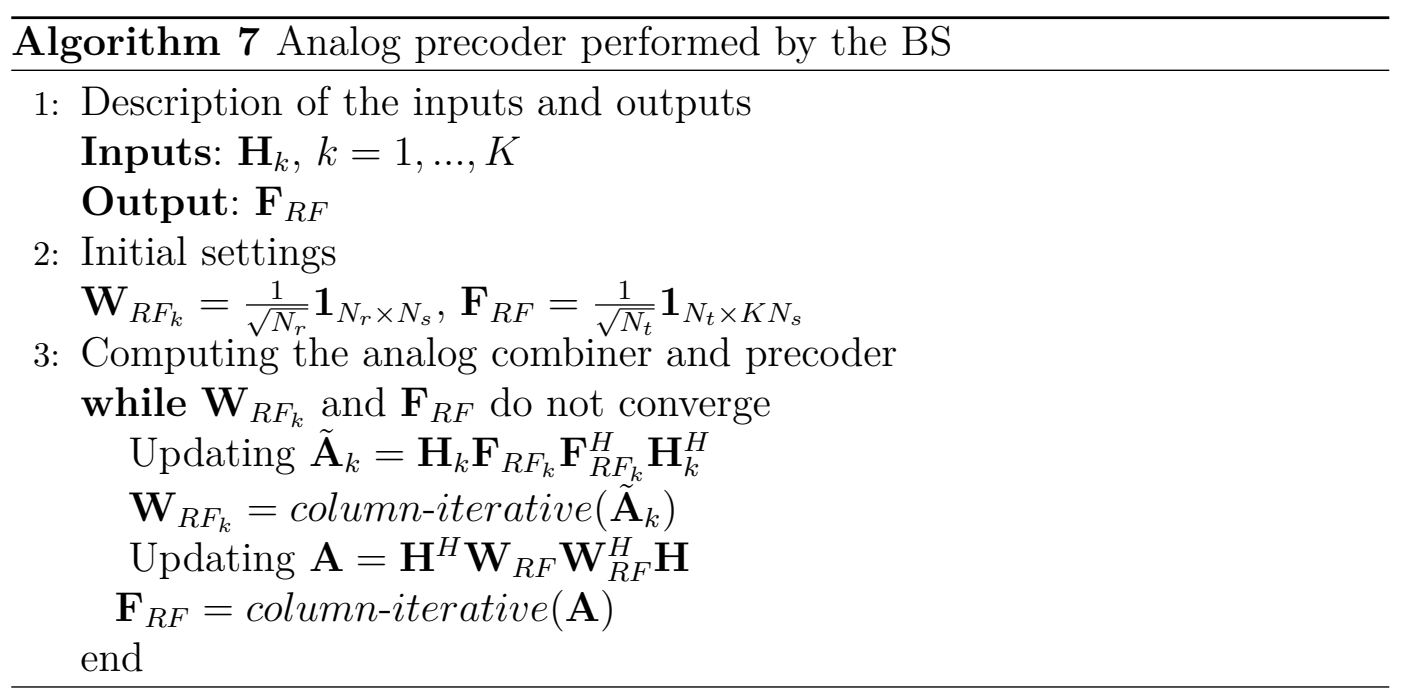

The procedure described in Algorithm 7 is performed by the BS. For the generation of the analog part of the combiner by the MS side, it is necessary that the receivers obtain an estimate of the product $\mathbf{H}_{k} \mathbf{F}_{R F_{k}}$ which can be simpler than obtaining an estimate of just $\mathbf{H}_{k}$. An option to obtain this estimate is by sending pilot symbols to a single user per time without the digital precoder part, such that the signal vector received by the user $k$ be

$$
\mathbf{r}_{k}=\mathbf{H}_{k} \mathbf{F}_{R F_{k}} \mathbf{s}_{k}+\mathbf{n}_{k}
$$

Then the MS can compute $\tilde{\mathbf{A}}_{k}=\mathbf{H}_{k} \mathbf{F}_{R F_{k}} \mathbf{F}_{R F_{k}}^{H} \mathbf{H}_{k}^{H}$ and finally $\mathbf{W}_{R F_{k}}=$ column-iterative $\left(\tilde{\mathbf{A}}_{k}\right)$. Note that considering an error-free estimation, the 
analog part of the precoder computed at the BS and at the MS are the same. In order not to increase the complexity of the hybrid combiner generation, the digital part of the hybrid combiner is considered as an identity matrix, i.e., $\mathbf{W}_{B B_{k}}=\mathbf{I}_{N_{s}}$.

For the digital part of the hybrid precoder the received signal vector processed by the user $k$ (see equation (2-2)) can be rewritten as

$$
\mathbf{y}_{k}=\dot{\mathbf{H}}_{k} \mathbf{F}_{R F} \mathbf{F}_{B B} \mathbf{S}+\dot{\mathbf{n}}
$$

where $\dot{\mathbf{H}}_{k}=\mathbf{W}_{B B_{k}}^{H} \mathbf{W}_{R F_{k}}^{H} \mathbf{H}_{k}$ and $\dot{\mathbf{n}} \sim \mathcal{C N}\left(\mathbf{0}, \mathbf{K}_{k}\right)$ with $\mathbf{K}_{k}=$ $\sigma_{n}^{2} \mathbf{W}_{B B_{k}}^{H} \mathbf{W}_{R F_{k}}^{H} \mathbf{W}_{R F_{k}} \mathbf{W}_{B B_{k}}$. For this model we propose the MMSE linear filter as derived in Appendix A.1.

$$
\mathbf{F}_{B B}=\left(\tilde{\mathbf{H}}^{H} \tilde{\mathbf{H}}+\frac{\gamma}{K N_{s}} \mathbf{F}_{R F}^{H} \mathbf{F}_{R F}\right)^{-1} \tilde{\mathbf{H}}^{H}
$$

where $\tilde{\mathbf{H}}=\left[\begin{array}{lll}\tilde{\mathbf{H}}_{1}^{T} & \cdots & \tilde{\mathbf{H}}_{K}^{T}\end{array}\right]^{T}$ with $\tilde{\mathbf{H}}_{k}=\dot{\mathbf{H}}_{k} \mathbf{F}_{R F}$, and $\gamma=\operatorname{tr}\{\mathbf{K}\}$ with $\mathbf{K}=\operatorname{blkdiag}\left\{\mathbf{K}_{1}, \ldots, \mathbf{K}_{K}\right\}[31]$.

\section{3}

\section{Hybrid precoder/combiner proposal II and III}

Our second and third proposals consist of the improvement of the digital part of the HB [21]-SVD-MMSE and [29]-CIA-MMSE, which are referred hereafter as P-SVD-MMSE* and P-CIA-MMSE*, respectively. This improvement is obtained through the replacement of the pseudo MMSE defined in the step 4 of Algorithm 3 by expression (4-7).

\section{4}

\section{Hybrid precoder/combiner proposal IV}

As previously mentioned the problem in the hybrid processing is the selection of the analog matrices $\mathbf{W}_{R F_{k}}$ and $\mathbf{F}_{R F}$. Since the goal is to reduce the inter user/symbol interference and also keeping SNR large, the ideal equivalent baseband channel of each user is a diagonal matrix with large entries. Consequently, obtaining an approximation for this diagonal matrix is a good approach for the design of the analog parts. The following subsections describe our fourth proposal for the hybrid combiner/precoder construction, which is referred hereafter as P-SVD*-MMSE*.

\subsection{1}

\section{Hybrid combiner proposal}

Based on (2-2) the mutual information between the information signal sent by the BS and the user $k$ can be written as follows 


$$
\mathbf{I}=\log _{2} \operatorname{det}\left(\mathbf{I}_{N_{s}}+\left(\sigma_{n}^{2} \mathbf{W}_{k}^{H} \mathbf{W}_{k}\right)^{-1} \mathbf{W}_{k}^{H} \mathbf{H}_{k} \mathbf{F} \mathbf{F}^{H} \mathbf{H}_{k}^{H} \mathbf{W}_{k}\right)
$$

where $\mathbf{W}_{k}=\mathbf{W}_{R F_{k}} \mathbf{W}_{B B_{k}}$ and $\mathbf{F}=\mathbf{F}_{R F} \mathbf{F}_{B B}$. One approach for the designing of the hybrid combiner is to find a couple of matrix, $\mathbf{W}_{B B_{k}}$ and $\mathbf{W}_{R F_{k}}$, that maximize (4-8) under the hardware constraints considered in mmWave scenarios. We then consider the optimization problem expressed by

$$
\begin{gathered}
\max _{\mathbf{W}_{R F_{k}}, \mathbf{W}_{B B_{k}}} \mathbf{I} \\
\text { s.t. }\left\|\left(\mathbf{W}_{R F_{k}}\right)_{i, j}\right\|=1 / \sqrt{N_{r}}
\end{gathered}
$$

Many methods have been proposed in the literature to solve (4-9), e.g., $[1,24,32]$, however, these methods involve complicated mathematical developments leading to complex solutions. In our approach we consider the approximation of the mutual information for large SNR.

$$
\tilde{\mathbf{I}}=\log _{2} \operatorname{det}\left(\left(\sigma_{n}^{2} \mathbf{W}_{k}^{H} \mathbf{W}_{k}\right)^{-1} \mathbf{W}_{k}^{H} \mathbf{H}_{k} \mathbf{F} \mathbf{F}^{H} \mathbf{H}_{k}^{H} \mathbf{W}_{k}\right)
$$

Then, using the properties of the determinant and taking (2-4) into account, (4-10) can be rewritten as:

$$
\tilde{\mathbf{I}}=\log _{2} \operatorname{det}\left(\operatorname{SNR}_{k}^{H} \mathbf{H}_{k} \overline{\mathbf{F}} \overline{\mathbf{F}}^{H} \mathbf{H}_{k}^{H} \mathbf{W}_{k}\right)-\log _{2} \operatorname{det}\left(\mathbf{W}_{k}^{H} \mathbf{W}_{k}\right)
$$

where $\overline{\mathbf{F}}=\frac{\mathbf{F}}{\|\mathbf{F}\|_{F}}$ and SNR $=\frac{E_{T}}{\sigma_{n}^{2}}$. Looking for a sub-optimum manageable solution for large SNR we concentrate in the channel dependent first term of (4-11) and disregard the second term. Furthermore, for practical issues the combiner design should not depend on the precoder knowledge, because the users do not have access to the whole precoder matrix, even with estimation procedures they can obtain only an estimate of their precoder part, $\mathbf{F}_{k}$. Therefore, we consider that $\overline{\mathbf{F}} \overline{\mathbf{F}}^{H} \propto \mathbf{I}_{N_{t}}$. This same assumption has been considered by other authors, e.g., [24]. With these simplifying assumptions the optimization problem is formulated as

$$
\begin{array}{r}
\max _{\mathbf{W}_{R F_{k}}, \mathbf{W}_{B B_{k}}} \operatorname{det}\left(\mathbf{W}_{k}^{H} \mathbf{H}_{k} \mathbf{H}_{k}^{H} \mathbf{W}_{k}\right) \\
\text { s.t. }\left\|\left(\mathbf{W}_{R F_{k}}\right)_{i, j}\right\|=1 / \sqrt{N_{r}}
\end{array}
$$

Note that a similar expression is considered in [24] (see equation (41)). The authors in [24] proposed a solution based on an iterative algorithm, whose complexity is considerable due to the matrix inversion required in each 
iteration (see Algorithm 4). From the Hadamard's inequality which states that if an arbitrary square matrix $\mathbf{A}$ is positive definite then

$$
\operatorname{det}(\mathbf{A}) \leq \prod_{i} \mathbf{A}_{i, i}
$$

with equality iff $\mathbf{A}$ is a diagonal matrix [36], it is desirable that the product $\mathbf{W}_{k}^{H} \mathbf{H}_{k} \mathbf{H}_{k}^{H} \mathbf{W}_{k}$ in (4-12) be a diagonal matrix with large entries. From the literature on traditional MIMO systems the solution of the expression above can easily be reached through SVD [30]. However, in mmWave scenarios this can not be used directly due to the constraints on the number of RF chains. To proceed with our proposed design, let us introduce the following SVD:

$$
\left[\mathbf{V}_{k}, \boldsymbol{\Lambda}_{k}, \mathbf{V}_{k}^{H}\right]=\operatorname{svd}\left(\mathbf{H}_{k} \mathbf{H}_{k}^{H}\right)
$$

We then construct the analog part of the combiner, $\mathbf{W}_{R F_{k}}$, from the $N_{R F_{r}}$ principal eigenvectors of $\mathbf{H}_{k} \mathbf{H}_{k}^{H}$ as follows

$$
\mathbf{W}_{R F_{k}}=\frac{1}{\sqrt{N_{r}}} \boldsymbol{\Psi}\left(\left(\mathbf{V}_{k}\right)_{:, 1: N_{R F_{r}}}\right)
$$

Note that $\Psi\left(\left(\mathbf{V}_{k}\right)_{:, 1: N_{R F_{r}}}\right)$ is an approximation of $\left(\mathbf{V}_{k}\right)_{:, 1: N_{R F_{r}}}$ when only phase shifters are used, which minimizes the euclidean distance between the unconstrained and phase constrained precoders/combiner [51]. Then, rewriting the problem in (4-12) in terms of the product between the user channel and analog combiner part, $\check{\mathbf{H}}_{k}=\mathbf{W}_{R F_{k}}^{H} \mathbf{H}_{k}$, we have

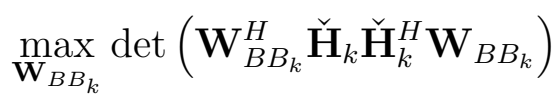

which represents a baseband problem similar to (4-12) but without constraints on the matrix construction, therefore, it can be solved using

$$
\mathbf{W}_{B B_{k}}=\left(\tilde{\mathbf{V}}_{k}\right)_{:, 1: N_{s}}
$$

where $\left[\check{\mathbf{V}}_{k}, \check{\Lambda}_{k}, \check{\mathbf{V}}_{k}^{H}\right]=\operatorname{svd}\left(\check{\mathbf{H}}_{k} \check{\mathbf{H}}_{k}^{H}\right)$. Algorithm 8 summarizes the steps for the realization of the proposed hybrid combiner.

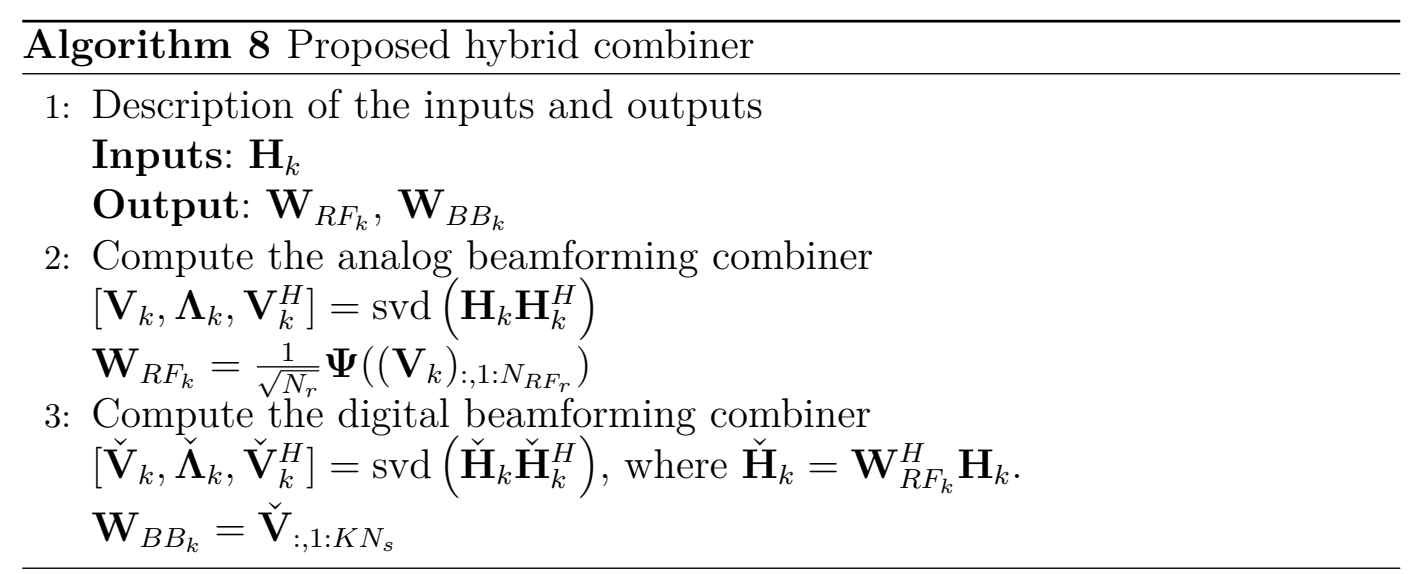




\subsection{2}

\section{Hybrid precoder proposal}

Our hybrid precoder design approach is divided in two parts, analog and digital. As stated in the previous subsection, the construction of the combiner's (precoder's) analog part based on the analog approximation to the eigenvectors of the channel user (entire channel) can benefit to diagonalization of the equivalent baseband user channel (entire equivalent baseband channel), which reduces the intersymbol (user) interference when is applied in the MS (BS). Thus, for the analog part of the precoder we consider the mutual information maximization problem of the entire channel, $\mathbf{H}=\left[\begin{array}{lll}\mathbf{H}_{1}^{T} & \cdots & \mathbf{H}_{K}^{T}\end{array}\right]^{T}$, when only analog processing is available in the transmitter, and we solve it through the same methodology used for the hybrid combiner realization.

Considering only analog processing in the transmitter with large SNR, the mutual information maximization problem of the entire channel can be reduced to the following problem

$$
\begin{aligned}
\max _{\mathbf{F}_{R F}} \operatorname{det} & \left(\mathbf{W}^{H} \mathbf{H} \mathbf{F}_{R F} \mathbf{F}_{R F}^{H} \mathbf{H}^{H} \mathbf{W}\right) \\
\text { s.t. } & \left\|\left(\mathbf{F}_{R F}\right)_{i, j}\right\|=1 / \sqrt{N_{t}}
\end{aligned}
$$

or equivalently as ${ }^{1}$.

$$
\begin{aligned}
\max _{\mathbf{F}_{R F}} & \left|\mathbf{F}_{R F}^{H} \mathbf{H}^{H} \mathbf{W} \mathbf{W}^{H} \mathbf{H} \mathbf{F}_{R F}\right| \\
& \text { s.t. }\left\|\left(\mathbf{F}_{R F}\right)_{i, j}\right\|=1 / \sqrt{N_{t}}
\end{aligned}
$$

where $\mathbf{W}=\operatorname{blkdiag}\left\{\mathbf{W}_{1}, \ldots, \mathbf{W}_{K}\right\}$. To construct $\mathbf{F}_{R F}$ we use the same methodology of the hybrid combiner described in Subsection 4.4.1. Therefore, the suboptimum analog part of the precoder is obtained as

$$
\mathbf{F}_{R F}=\frac{1}{\sqrt{N_{t}}} \boldsymbol{\Psi}\left(\mathbf{V}_{:, 1: N_{R F_{t}}}\right)
$$

where $\left[\mathbf{V}, \boldsymbol{\Lambda}, \mathbf{V}^{H}\right]=\operatorname{svd}\left(\mathbf{H}^{H} \mathbf{W} \mathbf{W}^{H} \mathbf{H}\right)$.

For the digital part of the hybrid precoder, low dimensional linear filters can be used, we considered the MMSE filter described in our first proposal, i.e., $\mathbf{F}_{B B}$ is given by (4-7). With $\mathbf{F}_{R F}$ given by (4-20) and $\mathbf{F}_{B B}$ obtained by (4-7), the product $\mathbf{F}_{R F} \mathbf{F}_{B B}$ is further normalized such that $\left\|\mathbf{F}_{R F} \mathbf{F}_{B B}\right\|_{F}^{2}=K N_{s}$

${ }^{1}$ Consider an arbitrary complex matrix $\mathbf{A} \in \mathcal{C}^{n \times m}$ with $n \leq m$. If $\mathbf{A A}^{H}$ is full ranking, then $\operatorname{det}\left(\mathbf{A} \mathbf{A}^{H}\right)=\prod_{i=1}^{n} \lambda_{i}=\left|\mathbf{A}^{H} \mathbf{A}\right|$, where $\lambda_{i}$ is the $i$-th eigenvalue of $\mathbf{A} \mathbf{A}^{H}$. 
Figure 4.1 resumes the main steps of the proposed methodology to obtain the analog precoder part and hybrid combiner. Note that the strategy applied on the hybrid combiner is very similar to the one employed in the hybrid precoder proposal described in Section 3.1 (see Figure3.1). One feature of that hybrid precoder is its poor interference capability as presented in Section 3.2. However, at the present hybrid precoder, this feature is improved by the MMSE performed in the digital part. Observe that the proposed methodology of the hybrid combiner design and analog precoder part are strongly related to each other, the maximization performed by the hybrid combiner indirectly increases the values of the cost function of the analog precoder part.

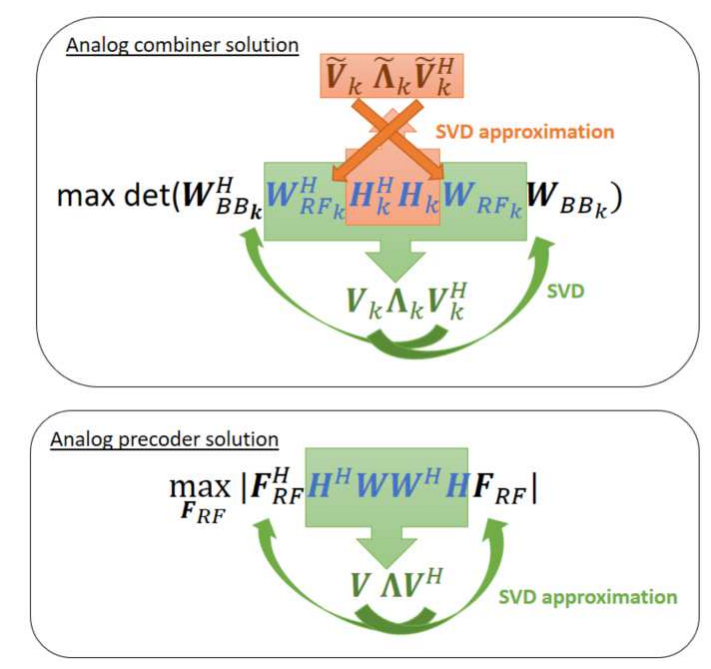

Figure 4.1: Design idea of the analog precoder and hybrid combiner

\section{5}

\section{Data detection approaches}

This section presents four sub-optimal approaches to obtain $\hat{\mathbf{s}}_{k}$ by each user requiring different levels of parameter knowledge (or estimation) as follows:

- Minimum distance detection (MDD)

$$
\hat{\mathbf{s}}_{k}=\underset{\mathbf{d} \in \mathbb{Q}^{N_{s} \times 1}}{\arg \min }\left\|\mathbf{y}_{k}-\mathbf{A}_{k} \mathbf{d}\right\|^{2}
$$

where $\mathbf{A}_{k}=\mathbf{W}_{B B_{k}}^{H} \mathbf{W}_{R F_{k}}^{H} \mathbf{H}_{k} \mathbf{F}_{H B_{k}}$, and $\mathbf{F}_{H B_{k}}$ is the, unknown to the receiver, submatrix of $\mathbf{F}_{R F} \mathbf{F}_{B B}$ corresponding to the hybrid precoder of user $k$.

- Approximate $M D D(A M D D)$ (assumes that $\mathbf{A}_{k} \approx \mathbf{I}\left(N_{s}\right)$ )

$$
\hat{\mathbf{s}}_{k}=\underset{\mathbf{d} \in \mathbb{Q}^{N_{s} \times 1}}{\arg \min }\left\|\tilde{\mathbf{y}}_{k}-\mathbf{d}\right\|^{2}
$$


- Noise whitening operation followed by MDD (NWMDD)

$$
\hat{\mathbf{s}}_{k}=\underset{\mathbf{d} \in \mathbb{Q}^{N_{s} \times 1}}{\arg \min }\left\|\mathbf{K}_{k}^{-1 / 2}\left(\mathbf{y}_{k}-\mathbf{A}_{k} \mathbf{d}\right)\right\|^{2}
$$

where $\mathbf{K}_{k}=\sigma_{n}^{2} \mathbf{W}_{k}^{H} \mathbf{W}_{k}$.

- Noise whitening operation followed by approximate MDD (NWAMDD)

$$
\hat{\mathbf{s}}_{k}=\underset{\mathbf{d} \in \mathbb{Q}^{N_{s} \times 1}}{\arg \min }\left\|\mathbf{K}_{k}^{-1 / 2}\left(\mathbf{y}_{k}-\mathbf{d}\right)\right\|^{2}
$$

- Noise and interference whitening operation followed by MDD (NWIMDD)

$$
\hat{\mathbf{s}}_{k}=\underset{\mathbf{d} \in \mathbb{Q}^{N_{s} \times 1}}{\arg \min }\left\|\mathbf{K}_{k}^{-1 / 2}\left(\mathbf{y}_{k}-\mathbf{A}_{k} \mathbf{d}\right)\right\|^{2}
$$

where $\mathbf{K}_{k}=\sigma_{n}^{2} \mathbf{W}_{k}^{H} \mathbf{W}_{k}+\sum_{\substack{j=1 \\ j \neq k}}^{K} \mathbf{W}_{k}^{H} \mathbf{H}_{k} \mathbf{F}_{j} \mathbf{F}_{j}^{H} \mathbf{H}_{k}^{H} \mathbf{W}_{k}$.

\section{6}

\section{Numerical results}

In the simulations, the users' channels are generated with $N_{p}=8$ multipath components, the azimuth and elevation departure angles values are given by a random variable with uniform distribution in the interval of $(0 ; 2 \pi)$ and $(0 ; \pi)$, respectively. The UPAs have square formats for both transmitter and receivers, i.e., $N_{t_{h}}=N_{t_{v}}=\sqrt{N}_{t}$ and $N_{r_{h}}=N_{r_{v}}=\sqrt{N}_{r}$. The maximum allowed setting for RF chains number is used for both the BS and for each MS, so that $N_{R F_{t}}=K N_{s}$ and $N_{R F_{r}}=N_{s}$. The results are averaged over $10^{5}$ channels generations for each user.

Reference [24] proposed an optimization involving the effective baseband user channel, $\mathbf{H}_{B B_{k}}=\mathbf{W}_{R F_{k}} \mathbf{H}_{k} \mathbf{F}_{R F}$, to find the analog part of the combiner/precoder. Their authors used the value of $\log _{2}\left(\prod_{i=1}^{K N_{s}} \lambda_{i}^{2}\right)$, where $\lambda_{i}$ is the $i$-th singular value of the entire effective baseband channel, $\mathbf{H}_{B B}=$ $\left[\begin{array}{lll}\mathbf{H}_{B B_{1}}^{T} & \cdots & \mathbf{H}_{B B_{K}}^{T}\end{array}\right]^{T}$, as the metric to ilustrate the advantages of their proposal. Figure 4.2 presents a comparison using this metric corresponding to the $\mathbf{H}_{B B}$ obtained by $[21,24]$ and our proposals. The simulation settings are $N_{t}=64, N_{r}=4, N_{s}=2$ and $K=4(K=8)$.

From Figure 4.2 it can be observed that [21]-SVD-MMSE and the hybrid designs proposed here yield the largest eigenvalues of the entire effective baseband channel. The results evidence that the considered SVD techniques can maximize the eigenvalues product due to their inherent capability of diagonalize a matrix. On other hand, despite the high complexity of P-CIA*MMSE* there is not a relevant gain if this metric is considered as a measure of performance. 


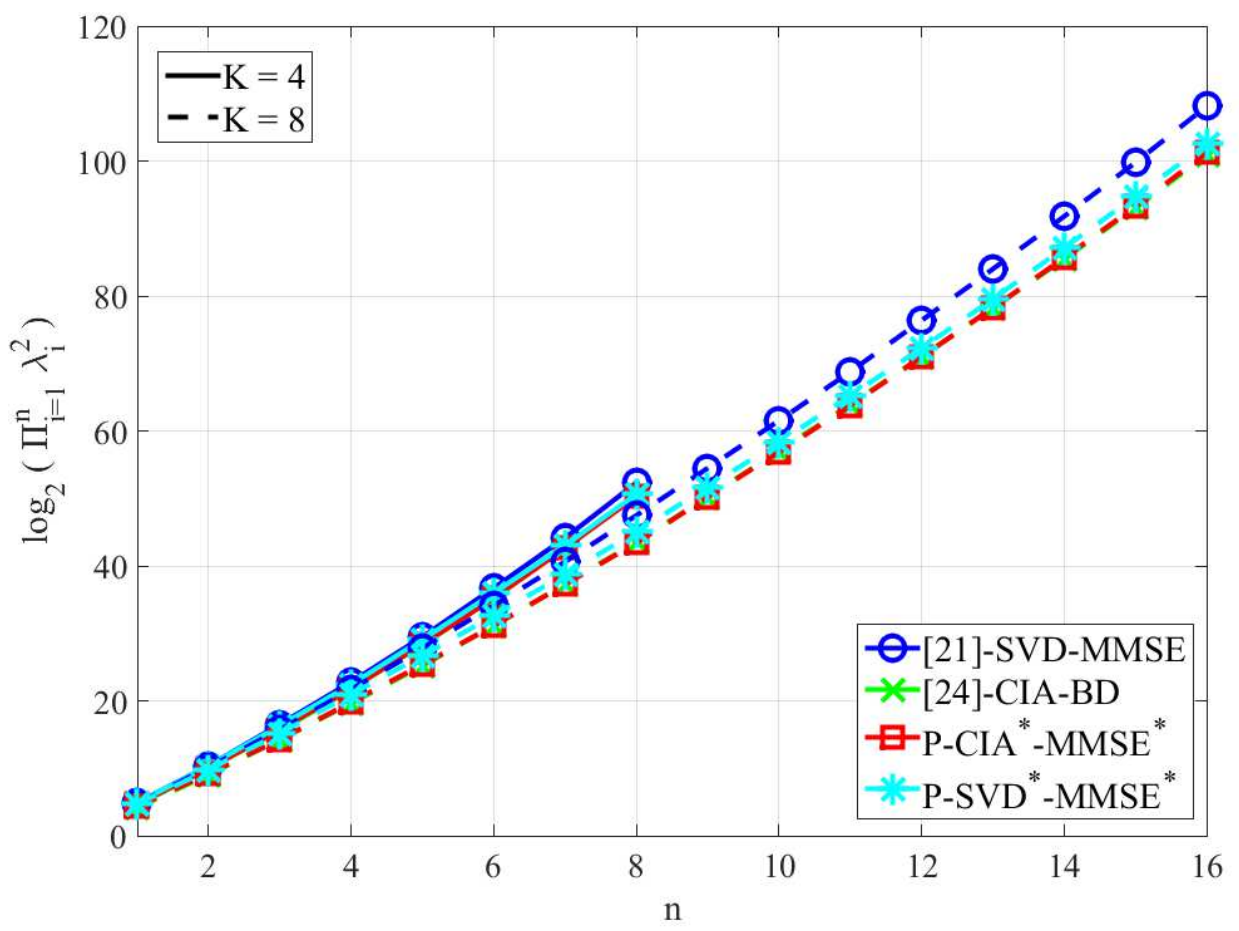

Figure 4.2: The value of $\log _{2}\left(\prod_{i=1}^{n} \lambda_{i}^{2}\right)$, with $\lambda_{1} \geq \lambda_{2} \geq \cdots \geq \lambda_{K N_{s}}$, of different equivalent baseband channels

Figure 4.3 shows a comparison in terms of sum rate computed by the expression

$$
\sum_{k=1}^{K} \log _{2} \operatorname{det}\left(\mathbf{I}_{N_{s}}+\mathbf{W}_{k}^{H} \mathbf{H}_{k} \mathbf{F}_{k} \mathbf{F}_{k}^{H} \mathbf{H}_{k}^{H} \mathbf{W}_{k} \mathbf{K}_{k}^{-1}\right)
$$

where $\mathbf{K}_{k}=\sigma_{n}^{2} \mathbf{W}_{k}^{H} \mathbf{W}_{k}+\sum_{\substack{j=1 \\ j \neq k}}^{K} \mathbf{W}_{k}^{H} \mathbf{H}_{k} \mathbf{F}_{j} \mathbf{F}_{j}^{H} \mathbf{H}_{k}^{H} \mathbf{W}_{k}$. In this figure the BS has $N_{t}=64$ antennas and sends $N_{s}=2$ streams to $K=4$ and $K=8$ users equipped with $N_{r}=4$.

From Figure 4.3, it can be observed that our proposal P-SVD*-MMSE* reaches the second-best sum rate performance for the two considered scenarios, while [24]-CIA-BD is the best one. In order to show the advantages of the proposed schemes in terms of BER performance we consider the following scenarios: in figures 4.4 and 4.5 the BS has $N_{t}=64$ antennas and sends $N_{s}=2$ streams to $K=4$ users, each equipped with $N_{r}=4$. In figures 4.6 and 4.7 the BS has $N_{t}=64$ antennas and sends $N_{s}=2$ streams to $K=8$ users equipped with $N_{r}=4$.

From the above mentioned figures it can be observed that the proposed P-SVD*-MMSE* achieved the lowest BER values in all considered scenarios and a simple data estimation, the AMDD, can be used without a relevant performance loss. Since mmWave systems is a promising solution to short 


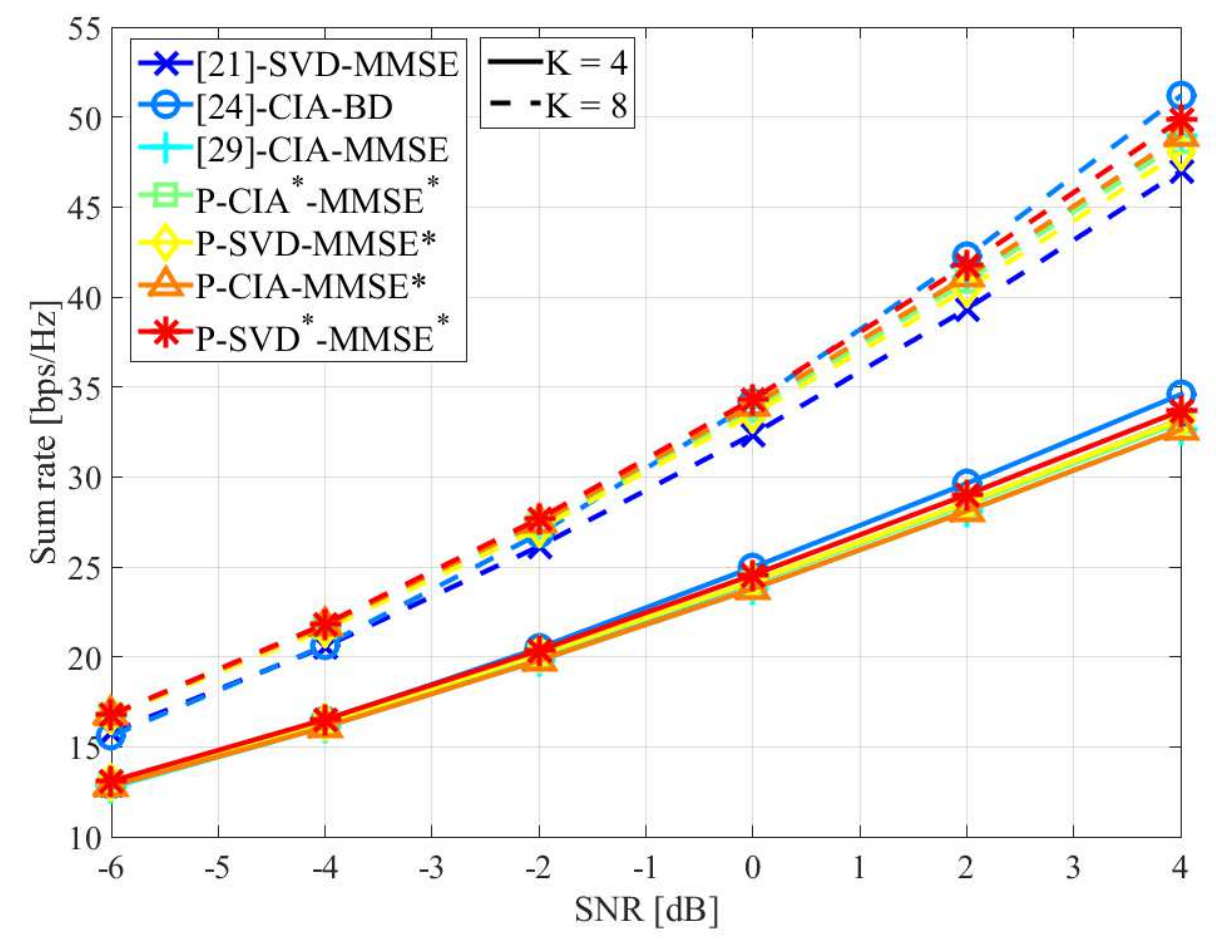

Figure 4.3: Achievable sum rate using $N_{t}=64, N_{r}=4, N_{s}=2, K=4$ and $K=8$

distance communications, simulations involving a number of users higher than 8 were not considered in this work.

Finally, Figures 4.8 and 4.9 illustrate the performance benchmark achieved by using NWIMDD in the terminals over the considered scenarios. 


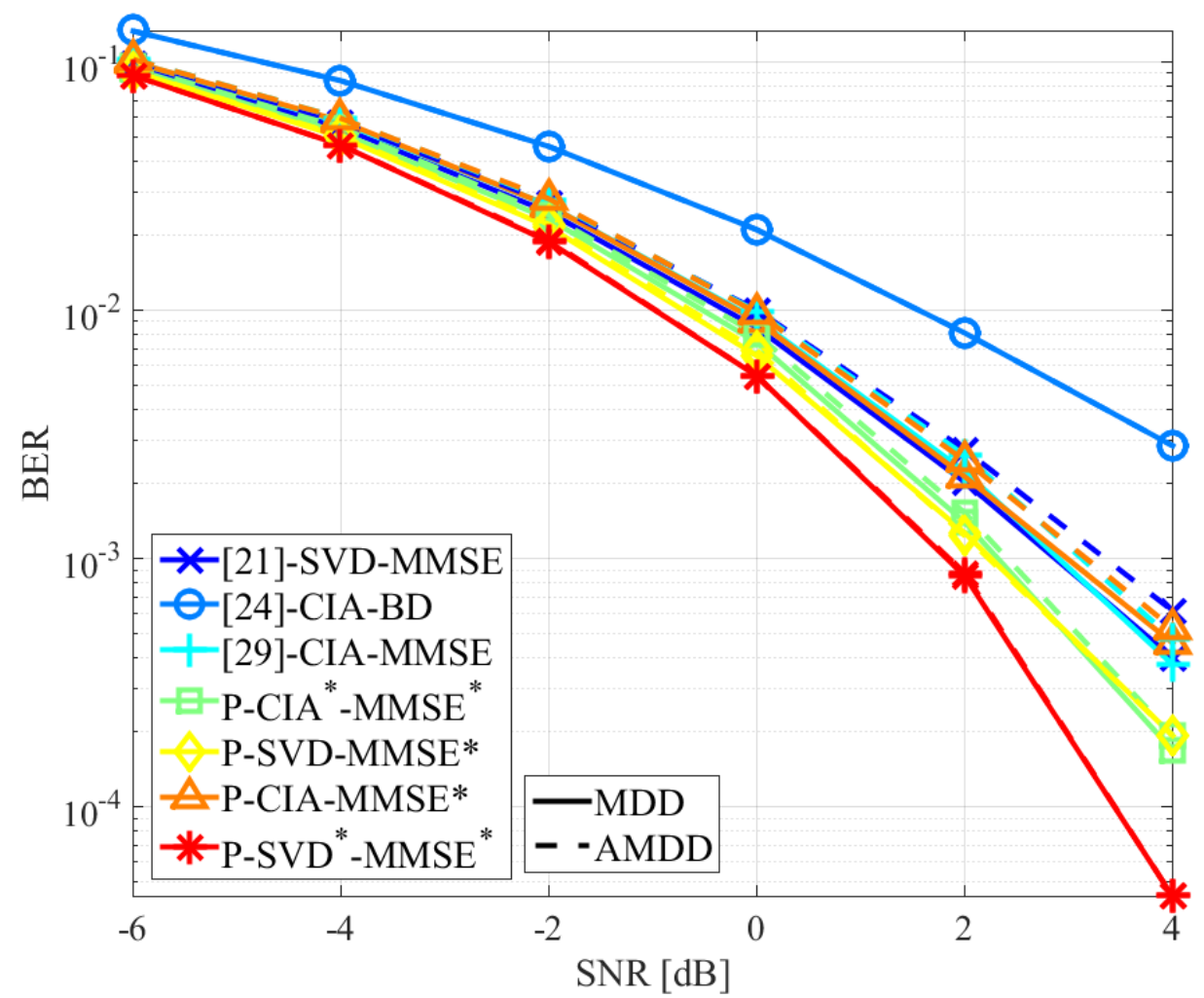

Figure 4.4: BER results obtained with the MDD and AMDD data detectors and simulation settings $N_{t}=64, N_{r}=4, N_{s}=2, K=4$ 


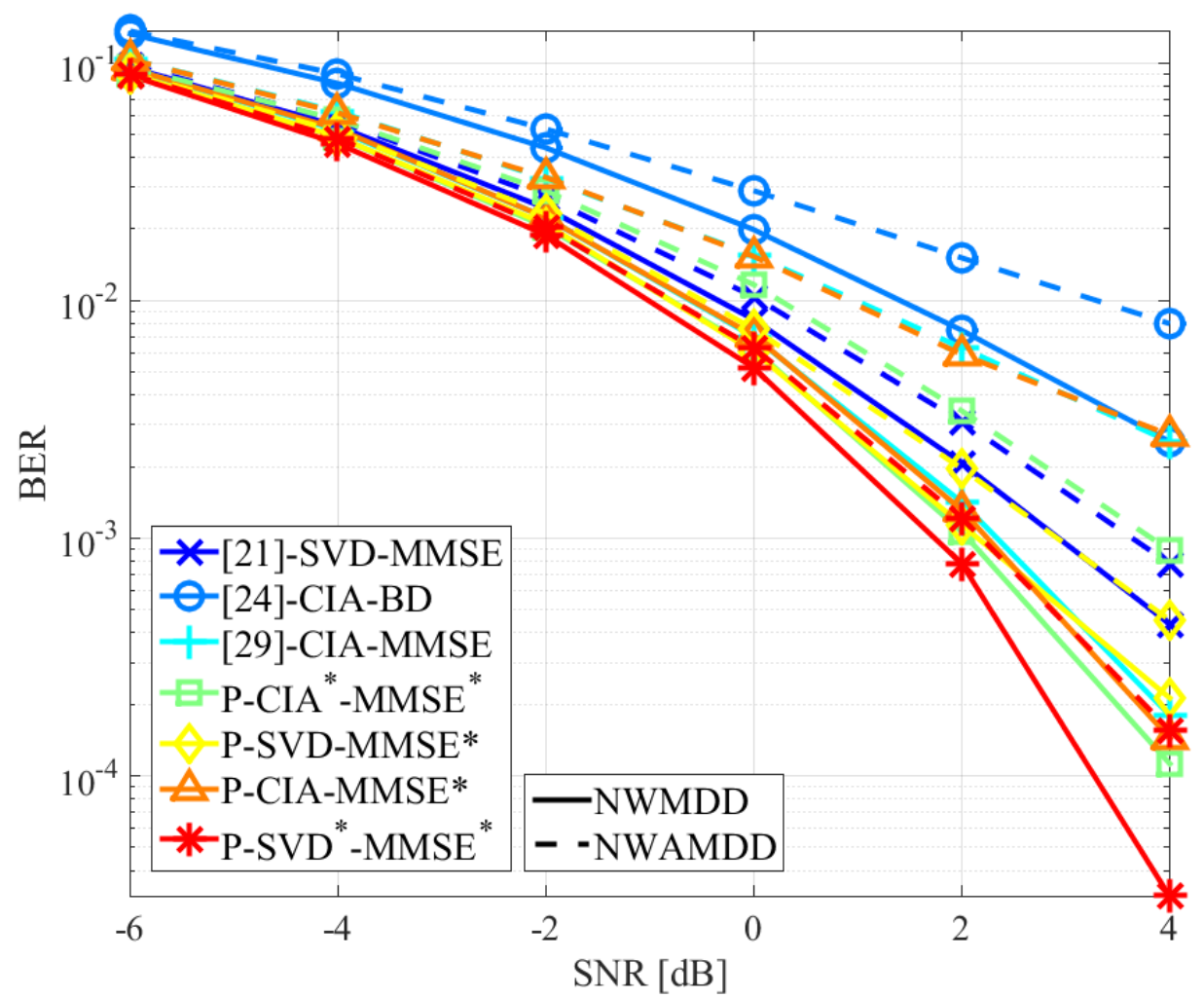

Figure 4.5: BER results obtained with the NWMDD and NWAMDD data detectors and simulation settings $N_{t}=64, N_{r}=4, N_{s}=2, K=4$ 


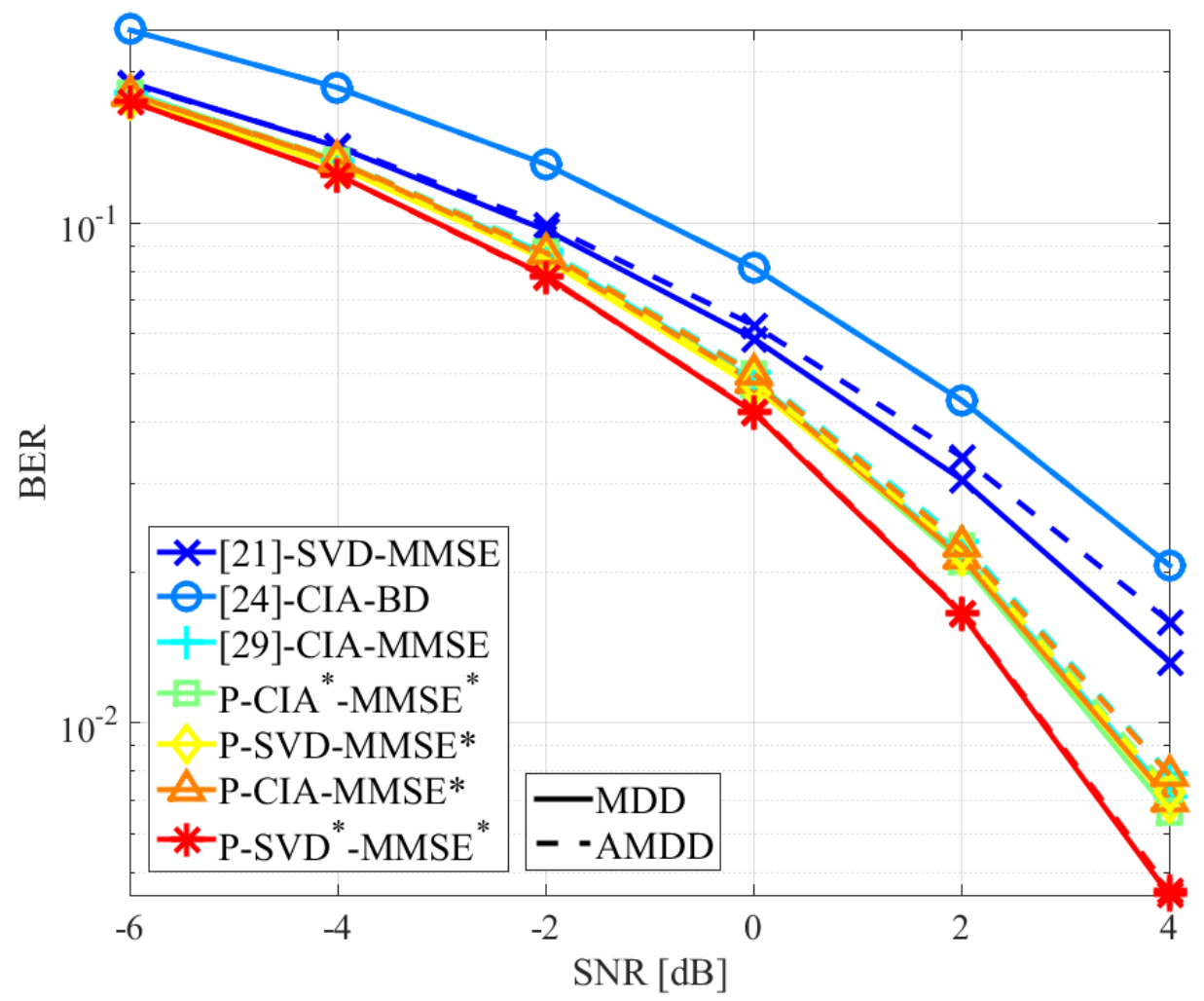

Figure 4.6: BER results obtained with the MDD and AMDD data detectors and simulation settings $N_{t}=64, N_{r}=4, N_{s}=2, K=8$ 


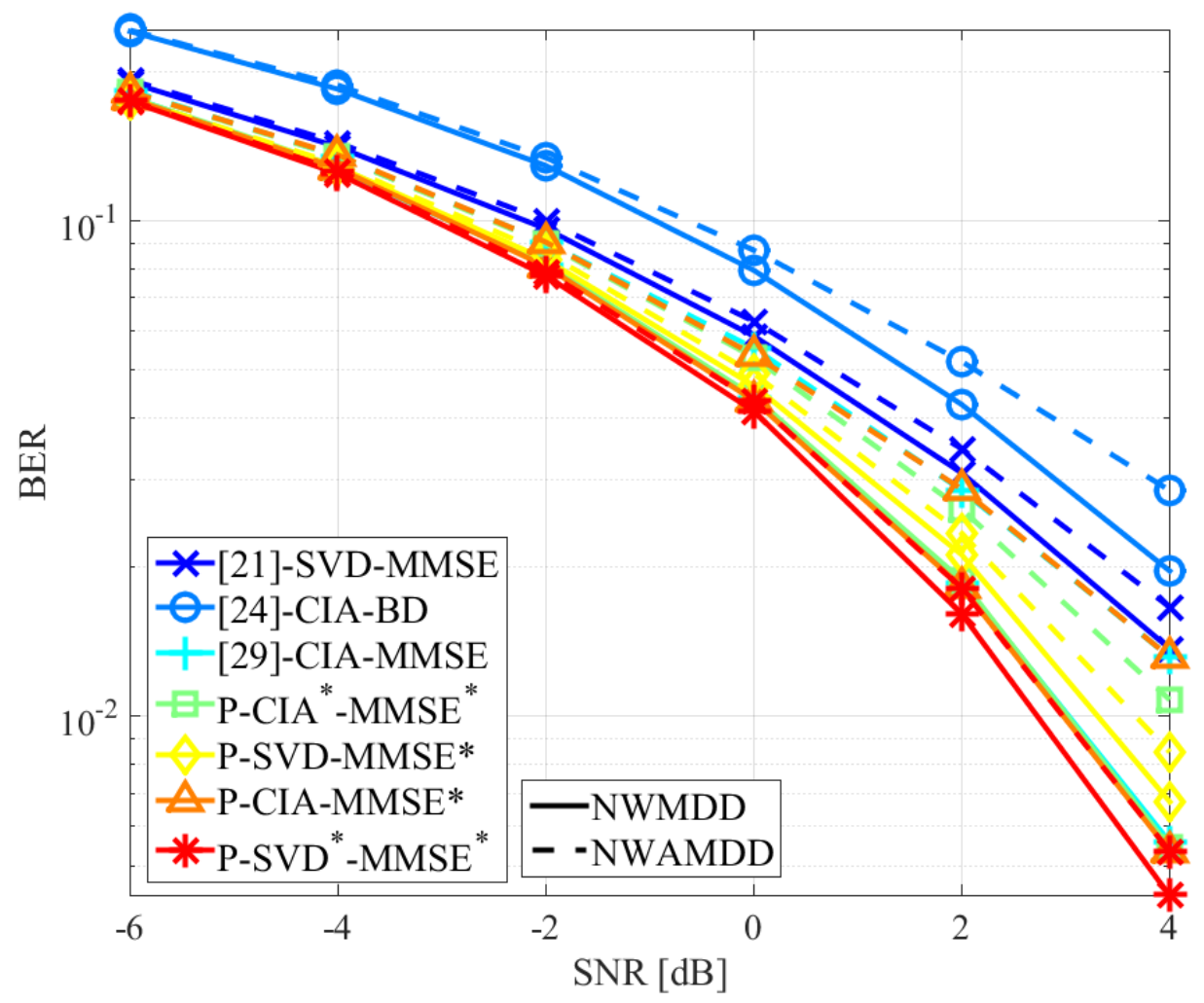

Figure 4.7: BER results obtained with the NWMDD and NWAMDD data detectors and simulation settings $N_{t}=64, N_{r}=4, N_{s}=2, K=8$

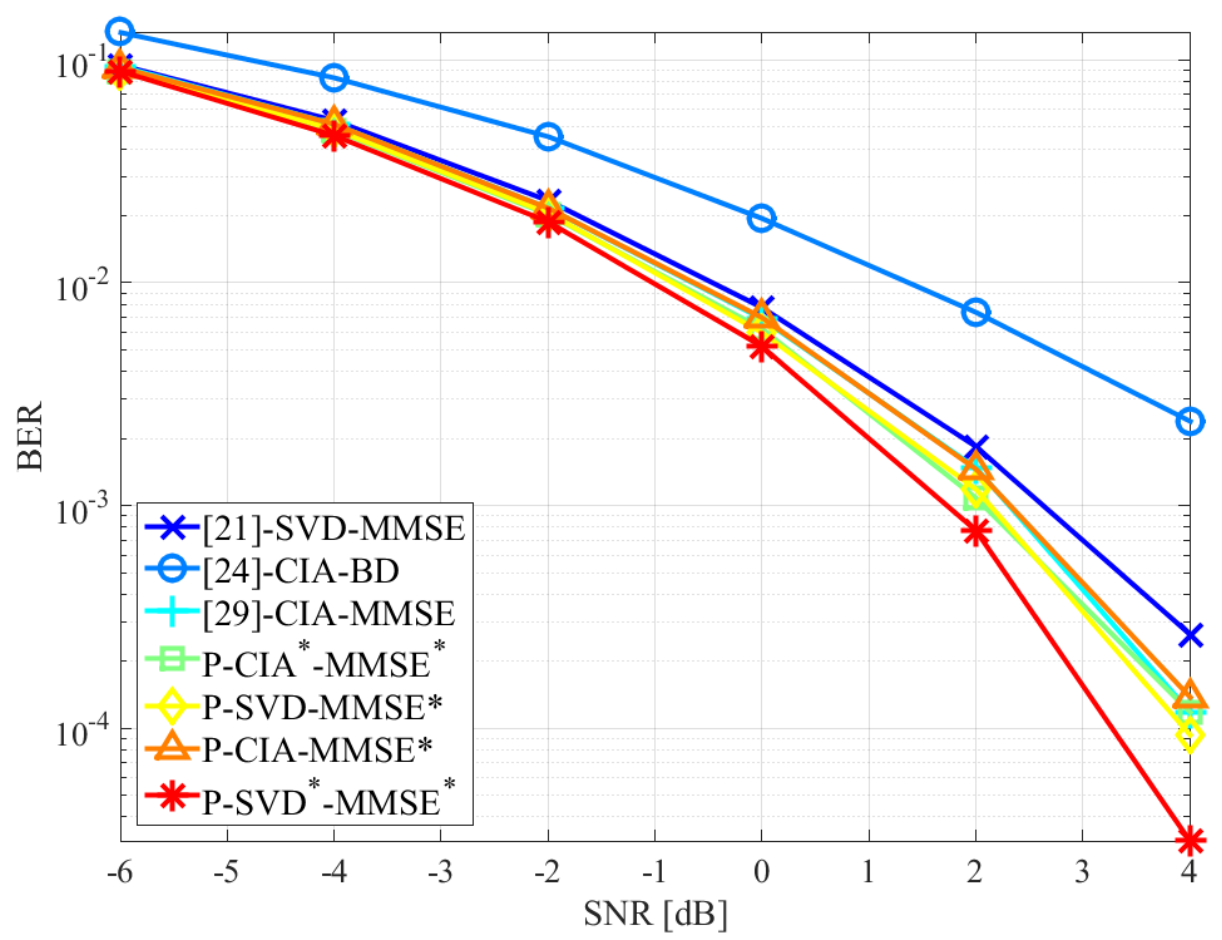

Figure 4.8: BER results obtained with the NWIMDD data detector and simulation settings $N_{t}=64, N_{r}=4, N_{s}=2, K=4$ 


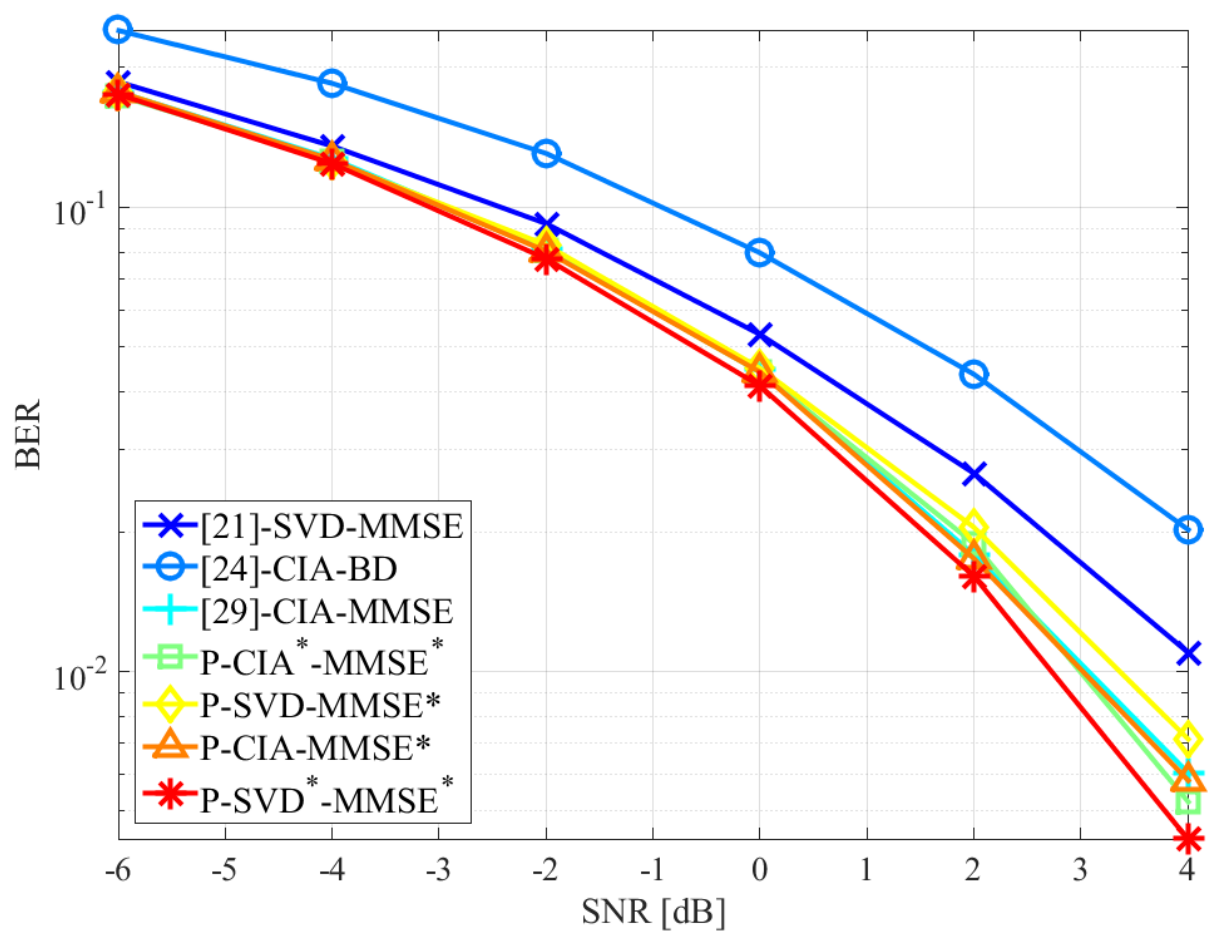

Figure 4.9: BER results obtained with the NWIMDD data detector and simulation settings $N_{t}=64, N_{r}=4, N_{s}=2, K=8$ 


\section{Method to find fairly tight analytic approximations to the BER performance}

Many hybrid beamforming designs have been proposed for the last years, e.g., $[21,24,29,39]$. However, to the best of our knowledge, there are no works presenting exact closed forms expressions for the BER performance in mmWave system. This chapter proposes a method to find fairly tight analytic approximations to the BER performance using different data detection approaches. The development of this methodology was based on [38], that describes a BER analysis for traditional MIMO. Adapting the methodology described in [38] to mmWave systems, would require the knowledge of the probability density function (pdf) of the variables involved, which are unknown for mmWave scenarios. Here, Gamma pdf approximations are used with support of [37], where expression to evaluate the accurateness of the Gamma/Gaussian approximation to an unknown random variable are presented. The analytic BER approximations resulted in differences no larger than $0.5 \mathrm{~dB}$ with respect to the simulation results in high SNR. In addition, a variation of our proposed P-SVD*-MMSE* described in Section 4.4 is included.

The remainder of this chapter is organized as follows: Section 5.1 describes some semi-analytic expressions to obtain an approximation to the BER performance; Section 5.2 presents our proposed methodology; and finally, in Section 5.4 numerical results obtained via simulation and through expressions are compared.

\section{1}

\section{Semi-analytic approximated BER expression}

The processed received signal can be rewritten as follows

$$
\begin{aligned}
\mathbf{y}_{k} & =\mathbf{W}_{k}^{H} \mathbf{H}_{k} \mathbf{F}_{k} \mathbf{s}_{k}+\sum_{j \neq k} \mathbf{W}_{k}^{H} \mathbf{H}_{k} \mathbf{F}_{j} \mathbf{s}_{j}+\mathbf{W}_{k}^{H} \mathbf{n}_{k} \\
& =\mathbf{B}_{k} \mathbf{s}_{k}+\tilde{\mathbf{n}}_{k}
\end{aligned}
$$

where $\mathbf{F}=\mathbf{F}_{R F} \mathbf{F}_{B B}=\left[\begin{array}{lll}\mathbf{F}_{1} & \cdots & \mathbf{F}_{K}\end{array}\right], \mathbf{W}_{k}=\mathbf{W}_{R F_{k}} \mathbf{W}_{B B_{k}}, \mathbf{B}_{k}=\mathbf{W}_{k}^{H} \mathbf{H}_{k} \mathbf{F}_{k}$ and $\tilde{\mathbf{n}}_{k}=\sum_{j \neq k} \mathbf{W}_{k}^{H} \mathbf{H}_{k} \mathbf{F}_{j} \mathbf{s}_{j}+\mathbf{W}_{k}^{H} \mathbf{n}_{k}$. The first term of $\tilde{\mathbf{n}}_{k}$ corresponds to the 
interference signal, and the second term represents the noise.

Consider a general scheme of data detection applied to (5-1) as follows

$$
\hat{\mathbf{s}}_{k}=\arg \min _{\mathbf{m} \in \mathbb{Q}^{N_{s}}}\left\|\mathbf{A}_{k}^{-1 / 2}\left(\mathbf{y}_{k}-\hat{\mathbf{B}}_{k} \mathbf{m}\right)\right\|^{2}
$$

where $\hat{\mathbf{B}}_{k}$ is an estimate of $\mathbf{B}_{k}$ and $\mathbf{A}_{k}$ is a Hermitian symmetric matrix given by the selected data detection approach. Some options for this matrix requiring different levels of parameter knowledge (or estimation) are described below.

- Minimum distance detection (MDD)

$$
\mathbf{A}_{k}=\mathbf{I}_{N_{s}}
$$

- Noise whitening operation followed by MDD (NWMDD)

$$
\mathbf{A}_{k}=\sigma_{n}^{2} \mathbf{W}_{k}^{H} \mathbf{W}_{k}
$$

- Noise and interference whitening operation followed by MDD (NI$W M D D)$

$$
\mathbf{A}_{k}=\tilde{\mathbf{K}}_{k}=\mathbb{E}\left[\tilde{\mathbf{n}}_{k} \tilde{\mathbf{n}}_{k}^{H}\right]=\sigma_{n}^{2} \mathbf{W}_{k}^{H} \mathbf{W}_{k}+\mathbf{K}_{\mathbf{i}_{\mathbf{k}}}
$$

where $\mathbf{K}_{\mathbf{i}_{\mathbf{k}}}=\mathbb{E}\left[\mathbf{i}_{k} \mathbf{i}_{k}^{H}\right]$, with $\mathbf{i}_{k}=\sum_{j \neq k} \mathbf{W}_{k}^{H} \mathbf{H}_{k} \mathbf{F}_{j} \mathbf{s}_{j}$.

Given the data detector in (5-2) and assuming that signal $\mathbf{s}_{k}$ was transmitted, then a symbol error occurs when $\hat{\mathbf{s}}_{k} \neq \mathbf{s}_{k}$, that is when

$$
\left\{\left\|\mathbf{A}_{k}^{-1 / 2}\left(\mathbf{y}_{k}-\hat{\mathbf{B}}_{k} \mathbf{s}_{k}\right)\right\|^{2}>\left\|\mathbf{A}_{k}^{-1 / 2}\left(\mathbf{y}_{k}-\hat{\mathbf{B}}_{k} \hat{\mathbf{s}}_{k}\right)\right\|^{2}\right\}
$$

Thereby a pairwise error event for user $k$ can be expressed as

$$
\begin{aligned}
\left\{\mathbf{s}_{i} \rightarrow \mathbf{s}_{j}\right\} & =\left\{\left\|\mathbf{A}_{k}^{-1 / 2}\left(\mathbf{y}_{k}-\hat{\mathbf{B}}_{k} \mathbf{s}_{i}\right)\right\|^{2}>\left\|\mathbf{A}_{k}^{-1 / 2}\left(\mathbf{y}_{k}-\hat{\mathbf{B}}_{k} \mathbf{s}_{j}\right)\right\|^{2}\right\} \\
& =\left\{-\mathbb{R}\left\{\left(\boldsymbol{\Delta}_{i i}^{(k)}-\boldsymbol{\Delta}_{i j}^{(k)}\right)^{H} \mathbf{A}_{k}^{-1} \tilde{\mathbf{n}}_{k}\right\}>\frac{\boldsymbol{\Delta}_{i j}^{(k) H} \mathbf{A}_{k}^{-1} \boldsymbol{\Delta}_{i j}^{(k)}-\boldsymbol{\Delta}_{i i}^{(k) H} \mathbf{A}_{k}^{-1} \boldsymbol{\Delta}_{i i}^{(k)}}{2}\right\}
\end{aligned}
$$

where $\boldsymbol{\Delta}_{i j}^{(k)}=\mathbf{B}_{k} \mathbf{s}_{i}-\hat{\mathbf{B}}_{k} \mathbf{s}_{j}$. If the interference term is a Gaussian vector $^{1}$ statistically independent of the noise vector with a covariance matrix $\mathbf{K}_{\mathbf{i}_{\mathbf{k}}}$; then

\footnotetext{
${ }^{1}$ In fact the interference term can be modeled as a Gaussian vector in a pessimistic case, i.e., large number of users, through the central limit theorem.
} 


$$
-\mathbb{R}\left\{\left(\boldsymbol{\Delta}_{i i}^{(k)}-\boldsymbol{\Delta}_{i j}^{(k)}\right)^{H} \mathbf{A}_{k}^{-1} \tilde{\mathbf{n}}_{k}\right\} \sim \mathcal{N}\left(\mathbf{0}, \frac{\left(\boldsymbol{\Delta}_{i i}^{(k)}-\boldsymbol{\Delta}_{i j}^{(k)}\right)^{H} \mathbf{A}_{k}^{-1} \tilde{\mathbf{K}}_{k} \mathbf{A}_{k}^{-1}\left(\boldsymbol{\Delta}_{i i}^{(k)}-\boldsymbol{\Delta}_{i j}^{(k)}\right)}{2}\right)
$$

where $\tilde{\mathbf{K}}_{k}$ given by (5-5). Therefore, the pairwise error probability, $P\left(\mathbf{s}_{i} \rightarrow \mathbf{s}_{j} \mid\right.$ $\left.\mathbf{A}_{k}, \mathbf{B}_{k}, \hat{\mathbf{B}}_{k}, \tilde{\mathbf{K}}_{k}\right)$, can be computed as follows

$P\left(\mathbf{s}_{i} \rightarrow \mathbf{s}_{j} \mid \mathbf{A}_{k}, \mathbf{B}_{k}, \hat{\mathbf{B}}_{k}, \tilde{\mathbf{K}}_{k}\right)=Q\left(\sqrt{\frac{\left(\boldsymbol{\Delta}_{i j}^{(k) H} \mathbf{A}_{k}^{-1} \boldsymbol{\Delta}_{i j}^{(k)}-\boldsymbol{\Delta}_{i i}^{(k) H} \mathbf{A}_{k}^{-1} \boldsymbol{\Delta}_{i i}^{(k)}\right)^{2}}{2\left(\boldsymbol{\Delta}_{i i}^{(k)}-\boldsymbol{\Delta}_{i j}^{(k)}\right)^{H} \mathbf{A}_{k}^{-1} \tilde{\mathbf{K}}_{k} \mathbf{A}_{k}^{-1}\left(\boldsymbol{\Delta}_{i i}^{(k)}-\boldsymbol{\Delta}_{i j}^{(k)}\right)}}\right)$

Using the upper bound to the probability of a union of events, the BER performance system is upper bounded by

$$
\mathrm{L}=c \sum_{k=1}^{K} \sum_{i=1}^{M} \sum_{j \neq i}^{M} d_{b_{i j}} \mathbb{E}\left[P\left(\mathbf{s}_{i} \rightarrow \mathbf{s}_{j} \mid \mathbf{A}_{k}, \mathbf{B}_{k}, \tilde{\mathbf{K}}_{k}\right)\right]
$$

where $c=\left(K M \log _{2}(M)\right)^{-1} ; M$ is the number of possible transmitted symbol vectors for the users; $d_{b_{i j}}$ is the Hamming distance between the equivalent bit representation of the symbol vectors $\mathbf{s}_{i}$ and $\mathbf{s}_{j}$, where $\mathbf{s}_{i} \in \mathbb{Q}^{N_{s}} \forall i$. It must be noted however that due to the Gaussian interference assumption, treating (5-10) as an upper bound is more appropriate in the case of a large number of users. In other cases it does not necessarily represent an upper bound, and is tested here as an approximation to the BER performance:

$\mathrm{BER} \approx L=c \sum_{k=1}^{K} \sum_{i=1}^{M} \sum_{j \neq i}^{M} d_{b_{i j}} \mathbb{E}\left[Q\left(\sqrt{\frac{\left(\boldsymbol{\Delta}_{i j}^{(k) H} \mathbf{A}_{k}^{-1} \boldsymbol{\Delta}_{i j}^{(k)}-\boldsymbol{\Delta}_{i i}^{(k) H} \mathbf{A}_{k}^{-1} \boldsymbol{\Delta}_{i i}^{(k)}\right)^{2}}{2\left(\boldsymbol{\Delta}_{i i}^{(k)}-\boldsymbol{\Delta}_{i j}^{(k)}\right)^{H} \mathbf{A}_{k}^{-1} \tilde{\mathbf{K}}_{k} \mathbf{A}_{k}^{-1}\left(\boldsymbol{\Delta}_{i i}^{(k)}-\boldsymbol{\Delta}_{i j}^{(k)}\right)}}\right)\right]$

The approximation (5-11), where the expected value in (5-11) is estimated by Monte Carlo method, i.e., the mean value is obtained over a large number of independent realizations of the random quantities involved, is referred hereafter as "Semi-analytic $\hat{\mathbf{B}}_{k}$ ".

Taking as particular case $\hat{\mathbf{B}}_{k}=\mathbf{B}_{k}$, then, (5-11) becomes

$$
\mathrm{BER} \approx c \sum_{k=1}^{K} \sum_{i=1}^{M} \sum_{j \neq i}^{M} d_{b_{i j}} \mathbb{E}\left[Q\left(\sqrt{\frac{\left(\mathbf{d}_{i j}^{H} \mathbf{B}_{k}^{H} \mathbf{A}_{k}^{-1} \mathbf{B}_{k} \mathbf{d}_{i j}\right)^{2}}{2 \mathbf{d}_{i j}^{H} \mathbf{B}_{k}^{H} \mathbf{A}_{k}^{-1} \tilde{\mathbf{K}}_{k} \mathbf{A}_{k}^{-1} \mathbf{B}_{k} \mathbf{d}_{i j}}}\right)\right]
$$

where $\mathbf{d}_{i j}=\mathbf{s}_{i}-\mathbf{s}_{j}$; and $\mathbf{s}_{i} \in \mathbb{Q}^{N_{s}} \forall i$. The Monte Carlo estimates obtained with $(5-12)$ are referred hereafter as "Semi-analytic $\mathbf{B}_{k}$ ". 


\section{2}

\section{Analytic approximate BER expression}

A further approximation explored here corresponds to evaluating the effects of the detection noise-plus-interference vector $\mathbf{n}_{d}=\mathbf{A}_{k}^{-1 / 2} \tilde{\mathbf{n}}_{k}$, with covariance matrix $\mathbf{A}^{-1 / 2} \tilde{\mathbf{K}}_{k} \mathbf{A}^{-1 / 2}$, by those of a white noise vector with the same total energy $\mathbb{E}\left[\left\|\mathbf{n}_{d}\right\|^{2}\right]$. We then replace matrix $\mathbf{A}_{k}^{-1 / 2} \tilde{\mathbf{K}}_{k} \mathbf{A}_{k}^{-1 / 2}$ in (5-12) by $\tilde{\sigma}_{k}^{2} \mathbf{I}_{N_{s}}$, where $\tilde{\sigma}_{k}^{2}=\frac{1}{N_{s}} \operatorname{tr}\left\{\tilde{\mathbf{K}}_{k} \mathbf{A}_{k}^{-1}\right\}$. (Note that such replacement is formally correct if $\mathbf{A}_{k}=\tilde{\mathbf{K}}_{k}$, as in the NIWMDD detection procedure). Approximation (5-12) then becomes

$$
\mathrm{BER} \approx c \sum_{k=1}^{K} \sum_{i=1}^{M} \sum_{j \neq i}^{M} d_{b_{i j}} \mathbb{E}\left[Q\left(\sqrt{\frac{\mathbf{d}_{k j}^{H} \mathbf{B}_{k}^{H} \mathbf{A}_{k}^{-1} \mathbf{B}_{k} \mathbf{d}_{k j}}{2 \tilde{\sigma}_{k}^{2}}}\right)\right]
$$

The Monte Carlo estimates obtained by (5-13) are referred hereafter as "Semi-analytic ". In addition, when the product $\mathbf{B}_{k}^{H} \mathbf{A}_{k}^{-1} \mathbf{B}_{k}$ is a diagonal matrix, then the system has no inter-symbol interference, and the detection in (5-2) can be made symbol by symbol separately, thus (5-13) can be written as

$$
\mathrm{BER} \approx \tilde{c} \sum_{k=1}^{K} \sum_{l=1}^{N_{s}} \sum_{i=1}^{N} \sum_{j \neq 1}^{N} \tilde{d}_{b_{i j}} \mathbb{E}\left[Q\left(\sqrt{\frac{\gamma_{l}^{(k)}\left\|\tilde{d}_{i j}\right\|^{2}}{2 \tilde{\sigma}_{k}^{2}}}\right)\right]
$$

where $\left(\gamma_{1}^{(k)}, \ldots, \gamma_{N_{s}}^{(k)}\right)=\operatorname{diag}\left(\mathbf{B}_{k}^{H} \mathbf{A}_{k}^{-1} \mathbf{B}_{k}\right) ; \tilde{c}=\left(K N N_{s} \log _{2}(N)\right)^{-1} ; N$ is the size of the signal constellation $\mathbb{Q} ; \tilde{d}_{b_{i j}}$ is the Hamming distance between the equivalent bit representation of the symbols of the constellation $s_{i}$ and $s_{j}$; $\tilde{d}_{i j}=s_{i}-s_{j}$; and $s_{i} \in \mathbb{Q}, \forall i$.

The computation of the expected value in (5-14) requires the knowledge of pdf of $\gamma_{l}^{(k)}$, which is mathematically challenging. However, the work in [37] describes a methodology to evaluate the accurateness of the Gamma/Gaussian approximation to an unknown pdf of a random variable. The authors of [37] present a couple of expressions for the approximation error, $E_{a p p}$, and for the maximum allowed error, $E_{u b}$. Thus, if $E_{a p p} \leq E_{u b}$ the approximation is considered as good.

A Gamma distribution can take only positive values, while Gaussian distribution takes positive and negative values. The selection between these two options is according to the considered problem. data. Since $\gamma_{l}^{(k)} \geq 0$, a Gamma pdf approximation is considered. This approximation has already being used in performance evaluations of traditional MIMO, e.g. [38]. Here, we considered the approximation $\gamma_{l}^{(k)} \sim \operatorname{gamma}\left(\alpha_{l}^{(k)}, \beta_{l}^{(k)}\right)$. The expression of the pdf of $\gamma_{l}$ is given by (we dropped the index $k$ for easiness of notation)

$$
f\left(\gamma_{l} \mid \alpha_{l}, \beta_{l}\right)= \begin{cases}\frac{\beta_{l}^{\alpha_{l}}}{\Gamma\left(\alpha_{l}\right)} \gamma_{l}^{\alpha_{l}-1} e^{-\beta_{l} \gamma_{l}}, & \gamma_{l} \geq 0 \\ 0, & \gamma_{l}<0\end{cases}
$$


where $\Gamma(\alpha)$ is the Gamma function defined as

$$
\Gamma(\alpha)=\int_{0}^{\infty} x^{\alpha-1} e^{-x} d x
$$

and the parameters $\alpha_{l}$ and $\beta_{l}$ are related as follows: $\mathbb{E}\left[\gamma_{l}\right]=m_{\gamma_{l}}=\frac{\alpha_{l}}{\beta_{1}}$, and $\mathbb{E}\left[\left(\gamma_{l}-m_{\gamma_{l}}\right)^{2}\right]=\sigma_{\gamma_{l}}^{2}=\frac{\alpha_{l}}{\beta_{l}^{2}}$.

Considering the fact that the $Q(\cdot)$ function is tightly upper bounded as $Q(x) \leq \frac{1}{6} e^{-2 x^{2}}+\frac{1}{12} e^{-x^{2}}+\frac{1}{4} e^{\frac{-x^{2}}{2}}[38]$. The expected value in (5-14) is upper bounded by

$$
\mathbb{E}\left[Q\left(\sqrt{b_{i j} \gamma_{l}}\right)\right] \leq \mathbb{E}\left[\frac{1}{6} e^{-2 b_{i j} \gamma_{l}}+\frac{1}{12} e^{-b_{i j} \gamma_{l}}+\frac{1}{4} e^{\frac{-b_{i j}}{2} \gamma_{l}}\right]
$$

where $b_{i j}=\frac{\left\|\tilde{d}_{i j}\right\|^{2}}{2 \tilde{\sigma}_{k}^{2}}$. Expression (5-17) requires the computation of expected values of the form $\mathbb{E}\left[a e^{-b \gamma}\right]$, where the index $l$ was dropped for easiness notation, derived next

$$
\begin{aligned}
\mathbb{E}\left[a e^{-b \gamma}\right] & =\int_{0}^{\infty} a e^{-b \gamma} \frac{\beta^{\alpha}}{\Gamma(\alpha)} \gamma^{\alpha-1} e^{-\beta \gamma} d_{\gamma} \\
& =a \int_{0}^{\infty} \frac{\beta^{\alpha}}{\Gamma(\alpha)} \gamma^{\alpha-1} e^{-(\beta+b) \gamma} d_{\gamma} \\
& =\frac{a \beta^{\alpha}}{(\beta+b)^{\alpha}} \int_{0}^{\infty} \frac{(\beta+b)^{\alpha}}{\Gamma(\alpha)} \gamma^{\alpha-1} e^{-(\beta+b) \gamma} d_{\gamma} \\
& =\frac{a \beta^{\alpha}}{(\beta+b)^{\alpha}}=g(a, b, \alpha, \beta)
\end{aligned}
$$

Note that $\int_{0}^{\infty} \frac{(\beta+b)^{\alpha}}{\Gamma(\alpha)} \gamma^{\alpha-1} e^{-(\beta+b) \gamma} d_{\gamma}=\int_{0}^{\infty} f(\gamma \mid \alpha, \beta+b) d_{\gamma}=1$ (see (5-15)). Using (5-18) in (5-17) yields

$$
\begin{aligned}
\mathbb{E}\left[Q\left(\sqrt{b_{i j} \gamma_{l}}\right)\right] \leq & g\left(\frac{1}{6}, 2 b_{i j}, \alpha_{l}, \beta_{l}\right)+ \\
& g\left(\frac{1}{12}, b_{i j}, \alpha_{l}, \beta_{l}\right)+g\left(\frac{1}{4}, \frac{b_{i j}}{2}, \alpha_{l}, \beta_{l}\right)
\end{aligned}
$$

\section{3}

\section{Hybrid design}

In this section we consider a variation of the hybrid beamforming PSVD*-MMSE* described in Chapter 4, that implements the well known Zero Forcing linear precoder as $\mathbf{F}_{B B}$. This proposed HB is referred hereafter as P-SVD*-ZF.

Algorithm 9 resumes the steps to generate the hybrid combiner and the analog part of the precoder is obtained by (5-20) according to Chapter 4 . 


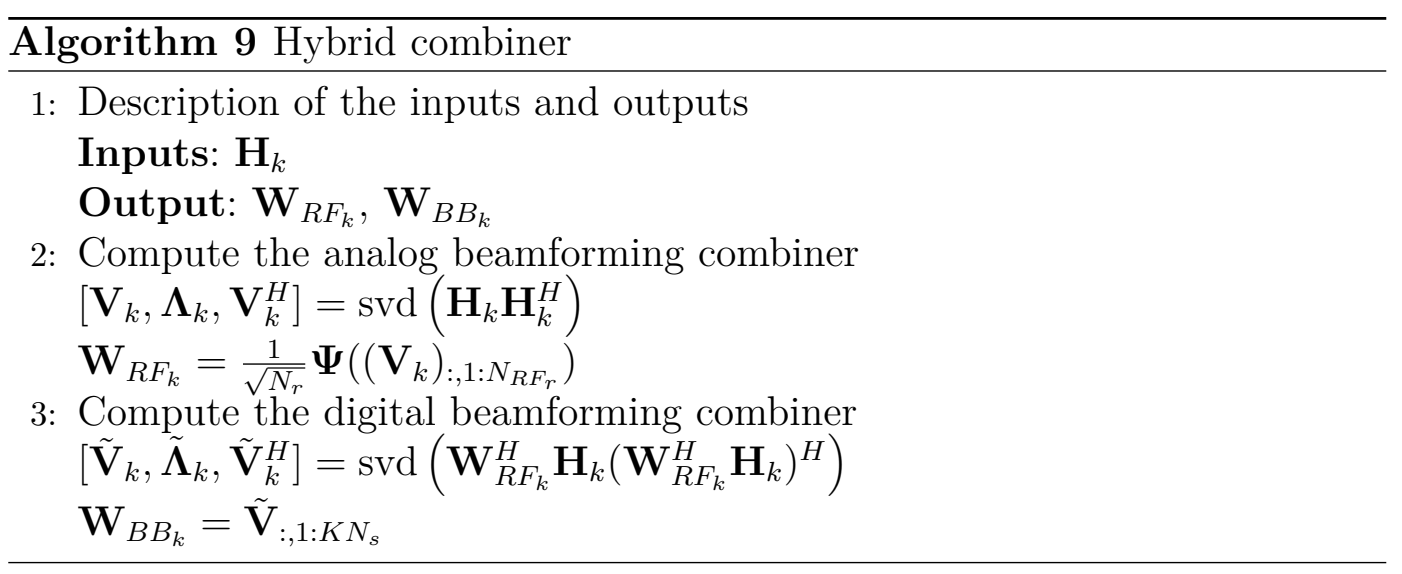

$$
\mathbf{F}_{R F}=\frac{1}{\sqrt{N_{t}}} \boldsymbol{\Psi}\left(\mathbf{V}_{:, 1: N_{R F_{t}}}\right)
$$

where $\left[\mathbf{V}, \boldsymbol{\Lambda}, \mathbf{V}^{H}\right]=\operatorname{svd}\left(\mathbf{H}^{H} \mathbf{W} \mathbf{W}^{H} \mathbf{H}\right)$, with $\mathbf{H}=\left[\begin{array}{lll}\mathbf{H}_{1}^{T} & \cdots & \mathbf{H}_{K}^{T}\end{array}\right]^{T}$ and $\mathbf{W}=\operatorname{blkdiag}\left(\mathbf{W}_{1}, \ldots, \mathbf{W}_{k}\right)$.

For the digital part of the hybrid precoder the ZF linear precoder is considered as function of the residual users channels, $\tilde{\mathbf{H}}_{k}=\mathbf{W}_{k}^{H} \mathbf{H}_{k} \mathbf{F}_{R F}$, as follows

$$
\mathbf{F}_{B B}=\tilde{\mathbf{H}}^{H}\left(\tilde{\mathbf{H}} \tilde{\mathbf{H}}^{H}\right)^{-1}
$$

where $\tilde{\mathbf{H}}=\left[\begin{array}{lll}\tilde{\mathbf{H}}_{1}^{T} & \cdots & \tilde{\mathbf{H}}_{K}^{T}\end{array}\right]^{T}$. Furthermore, in order to satisfy the power constraint, a normalization constant is included such that $\left\|\mathbf{F}_{R F} \mathbf{F}_{B B}\right\|_{F}^{2}=$ $K N_{s}$.

Note that when P-SVD*-ZF is used the interference term in (5-1) is eliminated, and therefore $\mathbf{K}_{i_{k}}=\mathbf{0}$.

\section{4}

\section{Numerical results}

In the simulations, the users' channels are generated with $N_{p}=10$ multipath components, the azimuth and elevation departure angles values are given by a random variable with uniform distribution in the interval of $(0,2 \pi)$ and $(0, \pi)$, respectively. The UPAs have square formats for both transmitter and receivers, i.e., $N_{t_{h}}=N_{t_{v}}={\sqrt{N_{t}}}_{\text {and }} N_{r_{h}}=N_{r_{v}}=\sqrt{N}_{r}$. The minimum allowed setting for RF chains number is used for both the BS and for each MS, so that $N_{R F_{t}}=K N_{s}$ and $N_{R F_{r}}=N_{s}$. The results are averaged over $10^{5}$ channel realizations for each user.

For Figures 5.4-5.10 two hybrid system are considered with the following characteristics: the BS has $N_{t}=64$ antennas and sends $N_{s}=2$ streams using modulation QPSK to $K=4(K=8)$ users equipped with $N_{r}=4\left(N_{r}=16\right)$. 
Figures 5.1 - 5.3 present BER simulation results obtained with the detection scheme in (5-2) and the BER values estimated by (5-11) using the three different options of $\mathbf{A}_{k}$ described in Section 5.1 and $\hat{\mathbf{B}}=\mathbf{I}_{N_{s}}$. Figure 5.1 shows the results considering [45]-SVD*-ZF, and Figures 5.2 and 5.3 considering P-SVD*-MMSE*.

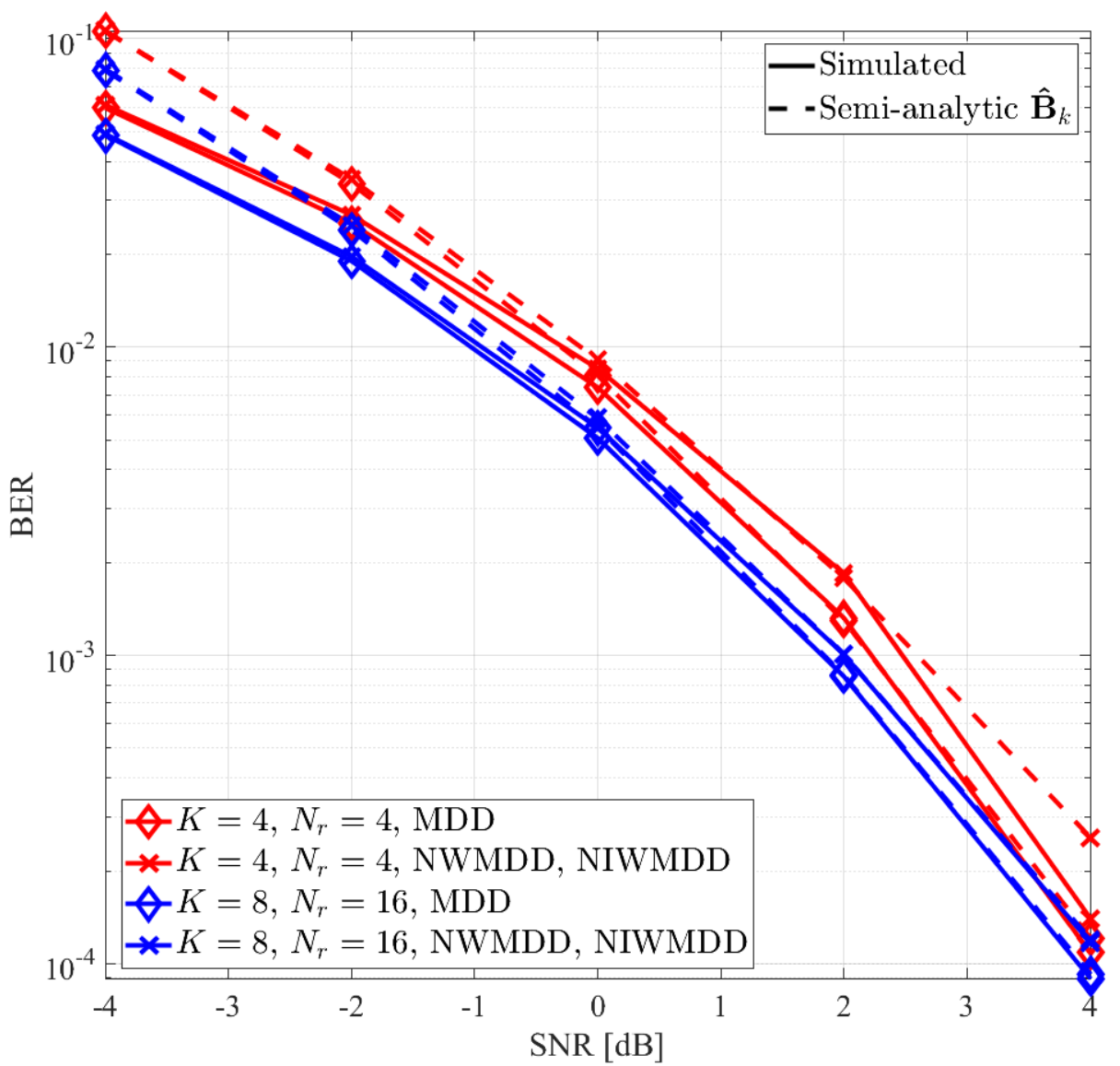

Figure 5.1: Simulated and estimated values of the BER performance using P-SVD*-ZF. Common simulation settings $N_{t}=64, N_{s}=2$ and $\hat{\mathbf{B}}=\mathbf{I}_{N_{s}}$

The usual hierarchical order of the different data detection schemes according to their BER performance, which is also related to their complexity, is NIWMDD, NWMDD and MDD, where NIWMDD is the best in terms of BER performance but the worst in terms of complexity. However, from Figures 5.1 - 5.3 it can be noted that when $\hat{\mathbf{B}}=\mathbf{I}_{N_{s}}$, which means $\hat{\mathbf{B}} \neq \mathbf{B}_{k}$, the hierarchical order changes to MDD, NWMDD and NIWMDD. The detection scheme presented in this text explores brute force to take out an estimate of the received data, where each candidate is rotated by the product $\mathbf{A}_{k}^{-1 / 2} \hat{\mathbf{B}}_{k}$ in (5-2) to obtain an alignment to the signal $\mathbf{A}_{k}^{-1 / 2} \mathbf{y}_{k}$. However when $\mathbf{A}_{k}^{-1 / 2} \hat{\mathbf{B}}_{k}=$ $\mathbf{A}_{k}^{-1 / 2}$, the resulting rotation could be rough even for P-SVD*-ZF, which has $\mathbf{B}_{k}=\mathbf{W}_{k} \mathbf{H}_{k} \mathbf{F}_{k} \propto \mathbf{I}_{N_{s}}$. 


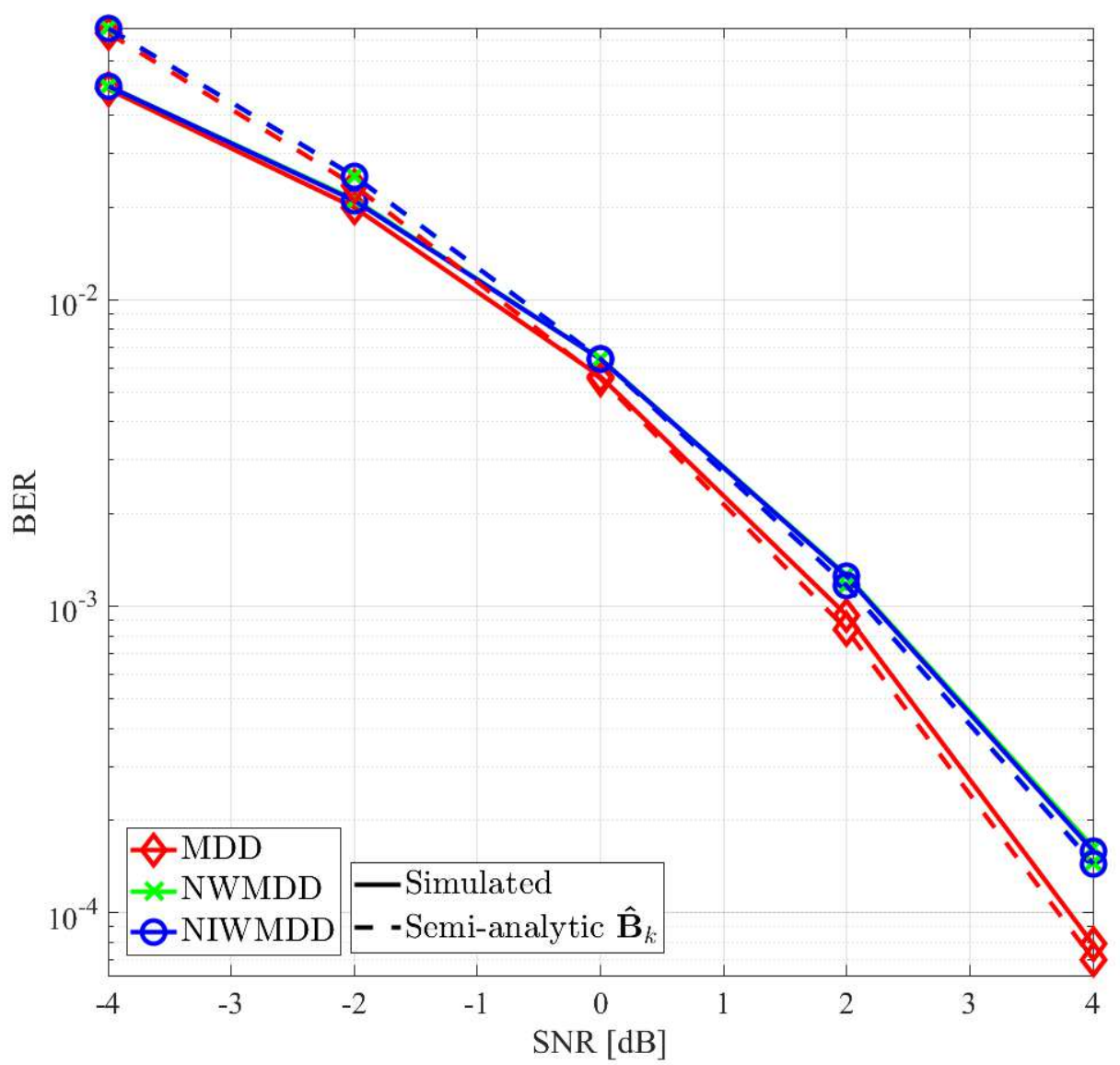

Figure 5.2: Simulated and estimated values of the BER performance using PSVD*-MMSE* $^{*}$ Simulation settings $N_{t}=64, N_{s}=2, K=4, N_{r}=4$, and $\hat{\mathbf{B}}=\mathbf{I}_{N_{s}}$

Consider the following notation: "Analytic $-N_{s}$ " computes the equation (5-14) using the $N_{s}$ random variables $\gamma_{l}^{(k)}$. "Analytic - 1" considers that $\gamma_{l}^{(k)}=\bar{\gamma}^{(k)}, l=1, \ldots, N s$, where $\bar{\gamma}^{(k)}=\frac{1}{N s} \sum_{l=1}^{N s} \gamma_{l}^{(k)}$ and uses (5-12). In this case (5-13) is used directly with $\mathbf{B}_{k}^{H} \mathbf{A}_{k}^{-1} \mathbf{B}_{k}=\bar{\gamma}^{(k)} \mathbf{I}_{N_{s}}$ and the expected value is computed using (5-19). Note that the Hamming distances in (5-13) can be larger than those in (5-14), resulting in lower values of the BER estimates obtained with "Analytic $-N_{s}$ " for low values of SNR. $\bar{d}_{b_{i, j}}$ is the Hamming distance between two symbol scalars, whereas $d_{b_{i, j}}$ is the Hamming distance between two symbol vectors.

Note that all expressions derived so far can be applied to an arbitrary modulation and precoding/combining [45]. However, if the modulation QPSK or BPSK is used, an approximation to the BER performance of the system is given by 


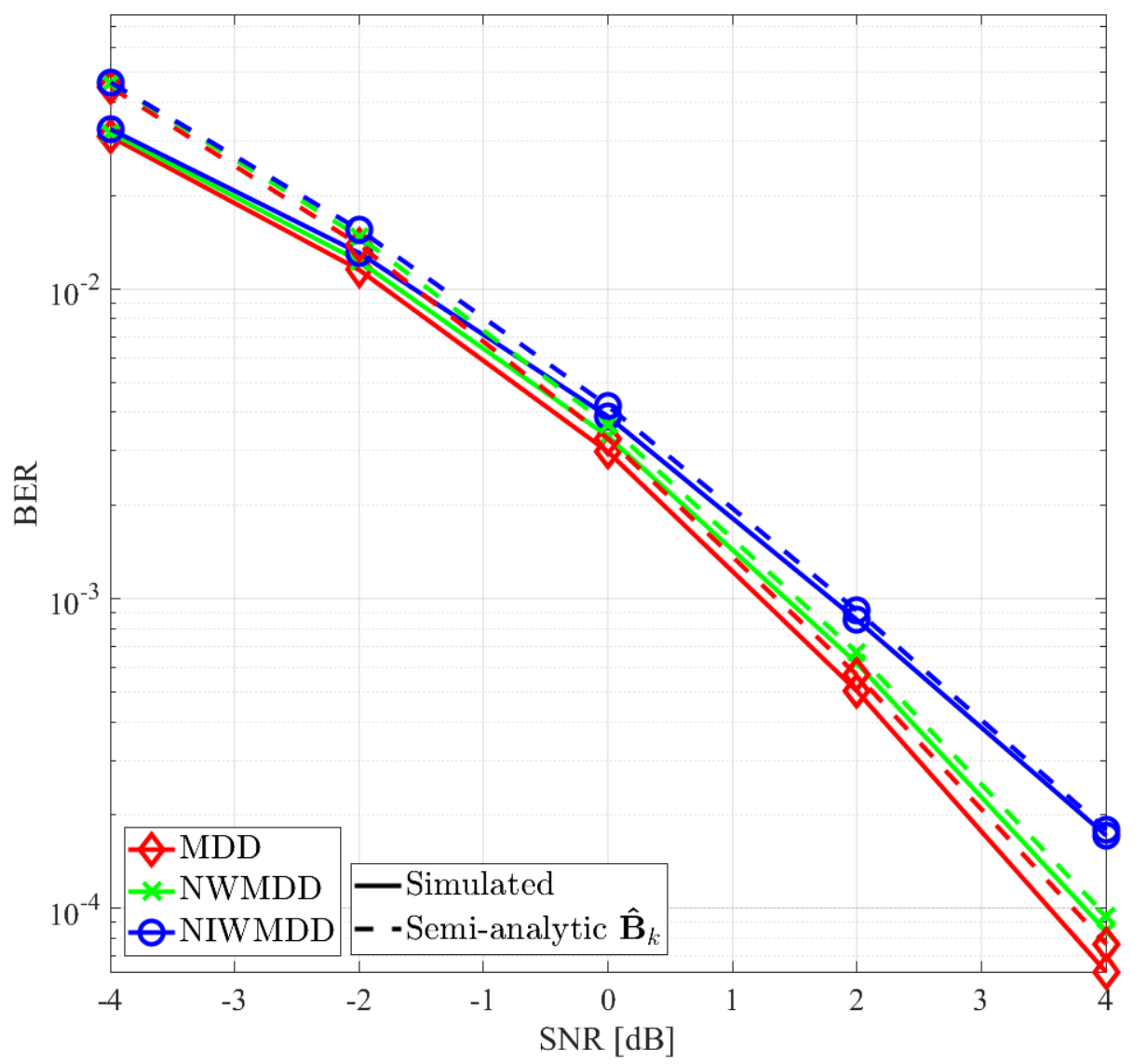

Figure 5.3: Simulated and estimated values of the BER performance using PSVD*-MMSE* ${ }^{*}$. Simulation settings $N_{t}=64, N_{s}=2, K=8, N_{r}=16$, and $\hat{\mathbf{B}}=\mathbf{I}_{N_{s}}$

$$
\mathrm{BER} \approx \frac{1}{K} \sum_{k=1}^{K} \mathbb{E}\left[Q\left(\sqrt{\frac{\bar{\gamma}^{(k)}}{\tilde{\sigma}_{k}^{2}}}\right)\right]
$$

The values of the BER performance estimated by (5-22) computing the expected value through (5-19) are referred as "Analytic - QPSK".

Figures 5.4 - 5.7 present the simulated and estimated values of the BER performance using P-SVD*-ZF resumed in Section 5.3 and the different data detectors considered.

From Figures 5.4 and 5.7, it can be observed that it does not exist a relevant difference between the BER performance results shown in Figures 5.4 and 5.5, probably because the product $\mathbf{W}_{k}^{H} \mathbf{W}_{k} \sim \mathbf{I}_{N_{s}}$, resulting in a noise term essentially white. Note that when P-SVD*-ZF is used the interference covariance matrix $\mathbf{K}_{i_{k}}=\mathbf{0}$, therefore in this case the BER performances obtained with both the NWMDD and the NIWMDD detection schemes are the same. 


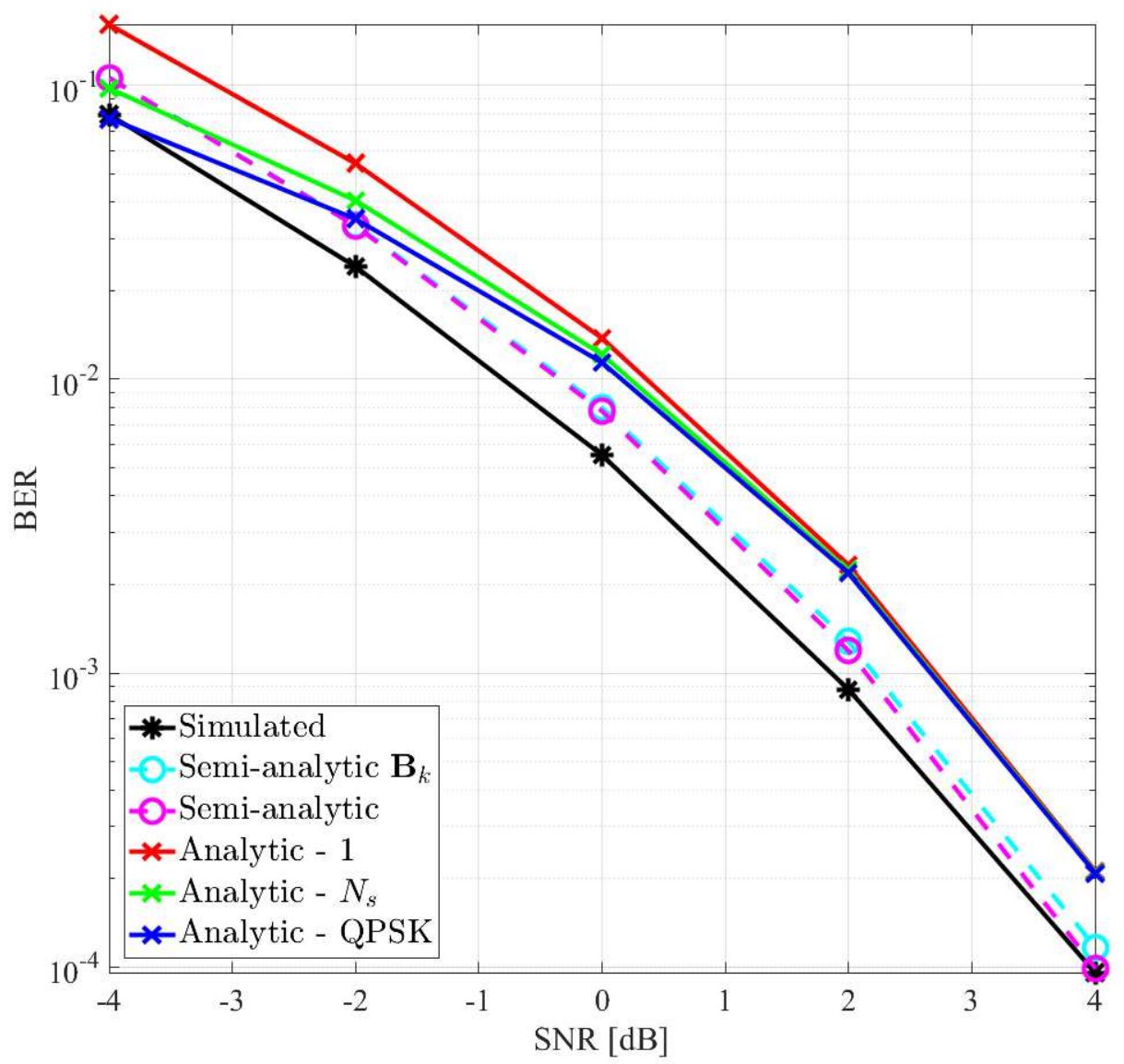

Figure 5.4: Simulated and estimated values of the BER performance using PSVD*-ZF. Simulation settings: MDD, $N_{t}=64, N_{s}=2, K=4, N_{r}=4$ and $\hat{\mathbf{B}}_{k}=\mathbf{B}_{k}$.

Figures 5.8 and 5.13 present the results of the BER performance of the P-SVD*-MMSE*, resumed in Section 5.3, using the considered data detectors and its semi-analytic approximations.

From Figures 5.4 - 5.13 it can be observed that all values estimated via Monte Carlo method are very close to the simulated values, specially when the SNR and number of users are high. On the other hand, the labeled "Analytic" expressions obtained with the proposed gamma approximation approach yield values very close to each other, and in high SNR they tend to the BER performance values obtained by simulation.

Tables 5.1, 5.2 and 5.3 present the mean, $m_{e}$, and variance, $\sigma_{e}^{2}$, of the absolute value of the BER differences, $\Delta_{\mathrm{BER}}$, between the approximations and simulations results presented in Figures 5.4-5.10.

From Figures 5.4-5.10 and Tables 5.1-5.3 it can be observed that "Analytic - QPSK" yields values closer to the simulated values than the other labeled "Analytic" expressions. 


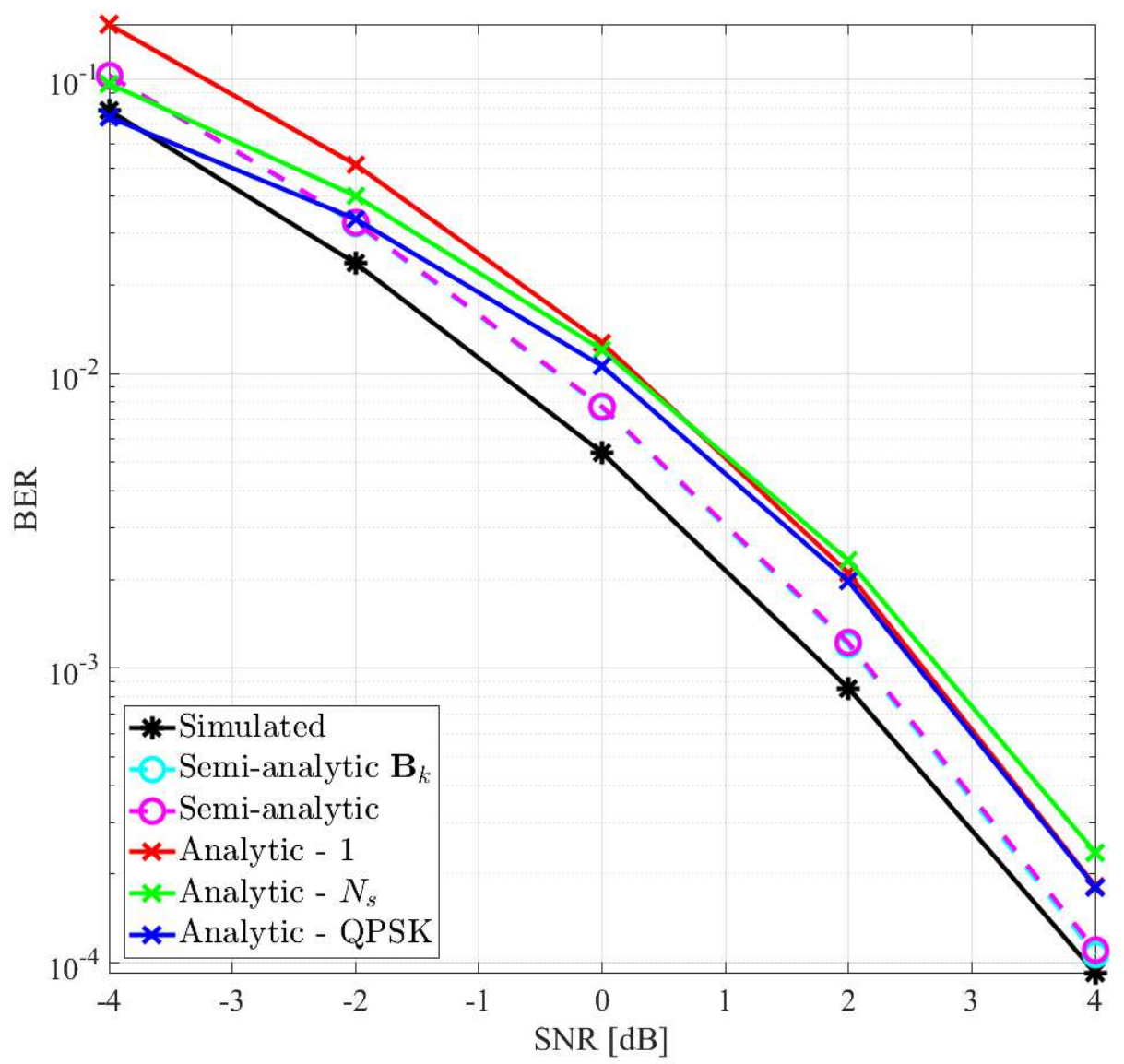

Figure 5.5: Simulated and estimated values of the BER performance using P-SVD*-ZF. Simulation settings: NWMDD (NIWMDD), $N_{t}=64, N_{s}=2$, $K=4, N_{r}=4$ and $\hat{\mathbf{B}}_{k}=\mathbf{B}_{k}$.

For Figure 5.14 the simulation settings are: the BS has $N_{t}=64$ antennas and sends $N_{s}=2$ streams using 16QAM to $K=4$ users equipped with $N_{r}=4$ using the NMDD to estimate the data.

For the settings adopted in this figure, the "Analytic $N_{s}$ " provided more accurate results than the other approximations, even more than the semianalytic expression for low SNR values. 


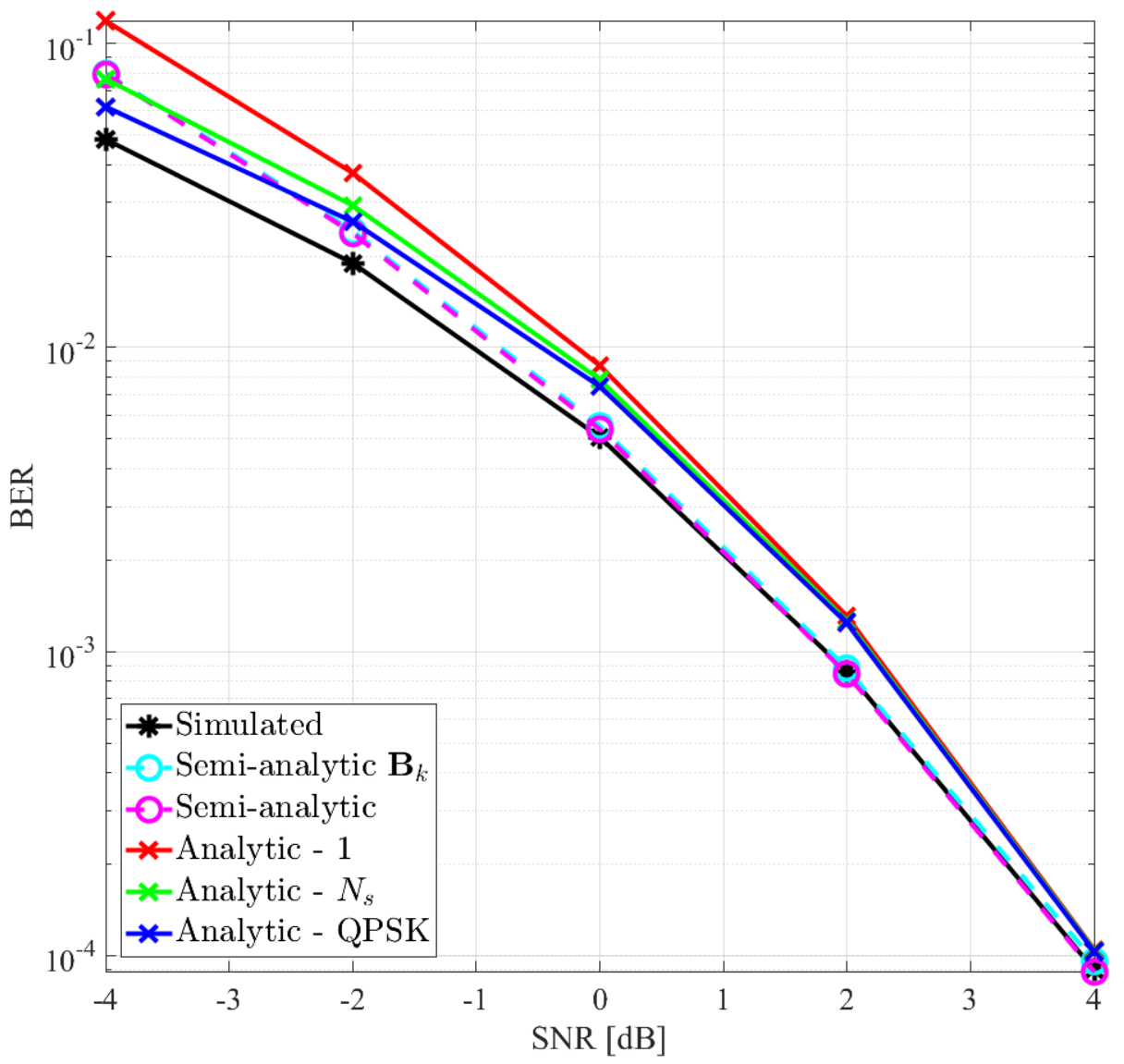

Figure 5.6: Simulated and estimated values of the BER performance using PSVD*-ZF. Simulation settings: MDD, $N_{t}=64, N_{s}=2, K=8, N_{r}=16$ and $\hat{\mathbf{B}}_{k}=\mathbf{B}_{k}$.

Table 5.1: Approximated BER accurateness in terms of mean and variance of the $\left|\Delta_{\text {BER }}\right|$ when MDD is used

\begin{tabular}{|c|c|c|c|c|c|c|c|c|}
\hline & \multicolumn{4}{|c|}{$K=4, N_{r}=4$} & \multicolumn{4}{|c|}{$K=8, N_{r}=16$} \\
\hline & \multicolumn{2}{|c|}{ P-SVD*-ZF } & \multicolumn{2}{|c|}{ P-SVD*-MMSE* } & \multicolumn{2}{|c|}{ P-SVD*-ZF } & \multicolumn{2}{|c|}{ P-SVD*-MMSE* } \\
\hline & $m_{e}$ & $\sigma_{e}^{2}$ & $m_{e}$ & $\sigma_{e}^{2}$ & $m_{e}$ & $\sigma_{e}^{2}$ & $m_{e}$ & $\sigma_{e}^{2}$ \\
\hline $\mathrm{SA} \mathbf{B}_{k}$ & $1.4837 \mathrm{e}-02$ & $6.0119 \mathrm{e}-04$ & $5.7392 \mathrm{e}-03$ & $1.1789 \mathrm{e}-04$ & $7.3386 \mathrm{e}-03$ & $1.8048 \mathrm{e}-04$ & $2.8468 \mathrm{e}-03$ & $2.7378 \mathrm{e}-05$ \\
\hline SA & $1.4683 \mathrm{e}-02$ & $6.0120 \mathrm{e}-04$ & $6.3818 \mathrm{e}-03$ & $1.3426 \mathrm{e}-04$ & $7.1008 \mathrm{e}-03$ & $1.7316 \mathrm{e}-04$ & $2.7049 \mathrm{e}-03$ & $2.6780 \mathrm{e}-05$ \\
\hline A - 1 & $3.1478 \mathrm{e}-02$ & $2.2710 \mathrm{e}-03$ & $1.4637 \mathrm{e}-02$ & $5.2060 \mathrm{e}-04$ & $1.8626 \mathrm{e}-02$ & $8.9905 \mathrm{e}-04$ & $7.4225 \mathrm{e}-03$ & $1.4934 \mathrm{e}-04$ \\
\hline $\mathrm{A}-N_{s}$ & $1.5666 \mathrm{e}-02$ & $4.2598 \mathrm{e}-04$ & $9.2341 \mathrm{e}-03$ & $1.5432 \mathrm{e}-04$ & $8.2921 \mathrm{e}-03$ & $1.3828 \mathrm{e}-04$ & $4.6289 \mathrm{e}-03$ & $4.5973 \mathrm{e}-05$ \\
\hline A - QPSK & $1.0250 \mathrm{e}-02$ & $1.4145 \mathrm{e}-04$ & $3.4420 \mathrm{e}-03$ & $1.3399 \mathrm{e}-05$ & $4.6304 \mathrm{e}-03$ & $3.1557 \mathrm{e}-05$ & $2.2315 \mathrm{e}-03$ & $8.2159 \mathrm{e}-06$ \\
\hline
\end{tabular}

Table 5.2: Approximated BER accurateness in terms of mean and variance of the $\left|\Delta_{\text {BER }}\right|$ when NWMDD is used

\begin{tabular}{|c|c|c|c|c|c|c|c|c|}
\hline & \multicolumn{4}{|c|}{$K=4, N_{r}=4$} & \multicolumn{4}{|c|}{$K=8, N_{r}=16$} \\
\hline & \multicolumn{2}{|c|}{ P-SVD*-ZF } & \multicolumn{2}{|c|}{ P-SVD*-MMSE* } & \multicolumn{2}{|c|}{ P-SVD*-ZF } & \multicolumn{2}{|c|}{ P-SVD*-MMSE* } \\
\hline & $m_{e}$ & $\sigma_{e}^{2}$ & $m_{e}$ & $\sigma_{e}^{2}$ & $m_{e}$ & $\sigma_{e}^{2}$ & $m_{e}$ & $\sigma_{e}^{2}$ \\
\hline $\mathrm{SA} \mathrm{B}_{k}$ & $1.4039 \mathrm{e}-02$ & $5.4305 \mathrm{e}-04$ & $5.3080 \mathrm{e}-03$ & $1.0208 \mathrm{e}-04$ & $7.0893 \mathrm{e}-03$ & $1.6976 \mathrm{e}-04$ & $2.7965 \mathrm{e}-03$ & $2.6511 \mathrm{e}-05$ \\
\hline SA & $1.4206 \mathrm{e}-02$ & $5.5247 \mathrm{e}-04$ & $5.6228 \mathrm{e}-03$ & $1.1270 \mathrm{e}-04$ & $6.9488 \mathrm{e}-03$ & $1.6505 \mathrm{e}-04$ & $2.5917 \mathrm{e}-03$ & $2.5040 \mathrm{e}-05$ \\
\hline A - 1 & $2.9256 \mathrm{e}-02$ & $1.9971 \mathrm{e}-03$ & $1.4444 \mathrm{e}-02$ & $5.1607 \mathrm{e}-04$ & $1.7929 \mathrm{e}-02$ & $8.4040 \mathrm{e}-04$ & $7.3757 \mathrm{e}-03$ & $1.4902 \mathrm{e}-04$ \\
\hline $\mathrm{A}-N_{s}$ & $1.5492 \mathrm{e}-02$ & $4.0614 \mathrm{e}-04$ & $8.0815 \mathrm{e}-03$ & $1.1969 \mathrm{e}-04$ & $8.1841 \mathrm{e}-03$ & $1.3300 \mathrm{e}-04$ & $4.3651 \mathrm{e}-03$ & $4.1481 \mathrm{e}-05$ \\
\hline A - QPSK & $9.4478 \mathrm{e}-03$ & $1.2276 \mathrm{e}-04$ & $3.3615 \mathrm{e}-03$ & $1.3167 \mathrm{e}-05$ & $4.3674 \mathrm{e}-03$ & $2.8228 \mathrm{e}-05$ & $2.2195 \mathrm{e}-03$ & $8.3526 \mathrm{e}-06$ \\
\hline
\end{tabular}




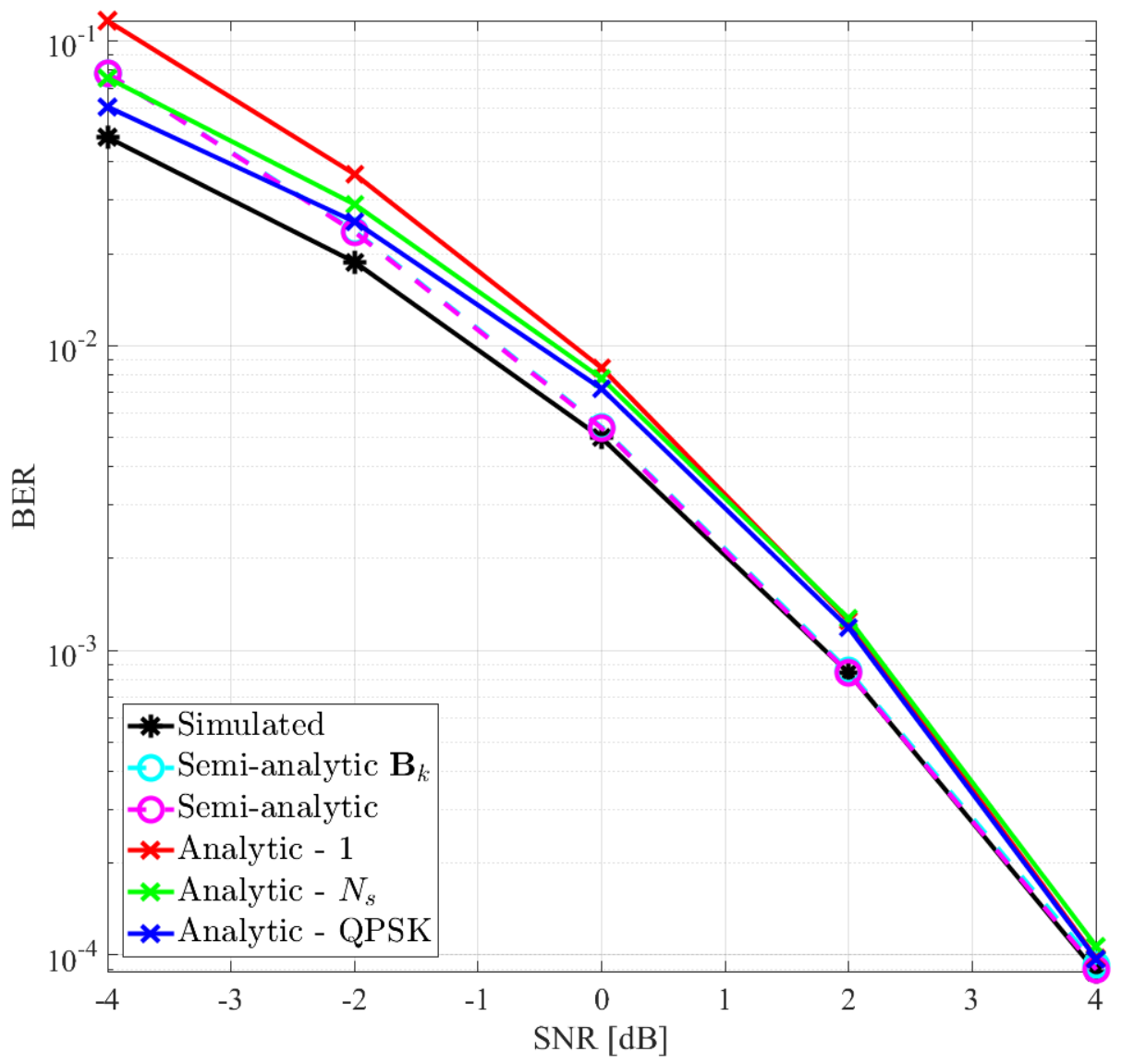

Figure 5.7: Simulated and estimated values of the BER performance using P-SVD*-ZF. Simulation settings: NWMDD (NIWMDD), $N_{t}=64, N_{s}=2$, $K=8, N_{r}=16$ and $\hat{\mathbf{B}}_{k}=\mathbf{B}_{k}$.

Table 5.3: Approximated BER accurateness in terms of mean and variance of the $\left|\Delta_{\text {BER }}\right|$ when NIMDD is used

\begin{tabular}{|c|c|c|c|c|c|c|c|c|}
\hline & \multicolumn{4}{|c|}{$K=4, N_{r}=4$} & \multicolumn{4}{|c|}{$K=8, N_{r}=16$} \\
\hline & \multicolumn{2}{|c|}{ P-SVD*-ZF } & \multicolumn{2}{|c|}{ P-SVD*-MMSE* } & \multicolumn{2}{|c|}{ P-SVD*-ZF } & \multicolumn{2}{|c|}{ P-SVD*-MMSE* } \\
\hline & $m_{e}$ & $\sigma_{e}^{2}$ & $m_{e}$ & $\sigma_{e}^{2}$ & $m_{e}$ & $\sigma_{e}^{2}$ & $m_{e}$ & $\sigma_{e}^{2}$ \\
\hline SA $\mathbf{B}_{k}$ & $1.4062 \mathrm{e}-02$ & $5.4424 \mathrm{e}-04$ & $5.2617 \mathrm{e}-03$ & $1.0041 \mathrm{e}-04$ & $7.0788 \mathrm{e}-03$ & $1.7143 \mathrm{e}-04$ & $2.4446 \mathrm{e}-03$ & $2.2160 \mathrm{e}-05$ \\
\hline SA & $1.4229 \mathrm{e}-02$ & $5.5365 \mathrm{e}-04$ & $5.6060 \mathrm{e}-03$ & $1.1160 \mathrm{e}-04$ & $7.0334 \mathrm{e}-03$ & $1.6986 \mathrm{e}-04$ & $2.4340 \mathrm{e}-03$ & $2.2092 \mathrm{e}-05$ \\
\hline A - 1 & $2.9278 \mathrm{e}-02$ & $1.9993 \mathrm{e}-03$ & $1.4194 \mathrm{e}-02$ & $4.9809 \mathrm{e}-04$ & $1.8061 \mathrm{e}-02$ & $8.5501 \mathrm{e}-04$ & $6.4830 \mathrm{e}-03$ & $1.1822 \mathrm{e}-04$ \\
\hline $\mathrm{A}-N_{s}$ & $1.5514 \mathrm{e}-02$ & $4.0720 \mathrm{e}-04$ & $8.1117 \mathrm{e}-03$ & $1.2012 \mathrm{e}-04$ & $8.2523 \mathrm{e}-03$ & $1.3637 \mathrm{e}-04$ & $4.2231 \mathrm{e}-03$ & $3.8882 \mathrm{e}-05$ \\
\hline A - QPSK & $9.4704 \mathrm{e}-03$ & $1.2335 \mathrm{e}-04$ & $3.2725 \mathrm{e}-03$ & $1.2329 \mathrm{e}-05$ & $4.4148 \mathrm{e}-03$ & $2.9370 \mathrm{e}-05$ & $1.8389 \mathrm{e}-03$ & $5.8644 \mathrm{e}-06$ \\
\hline
\end{tabular}


Chapter 5. Method to find fairly tight analytic approximations to the BER performance

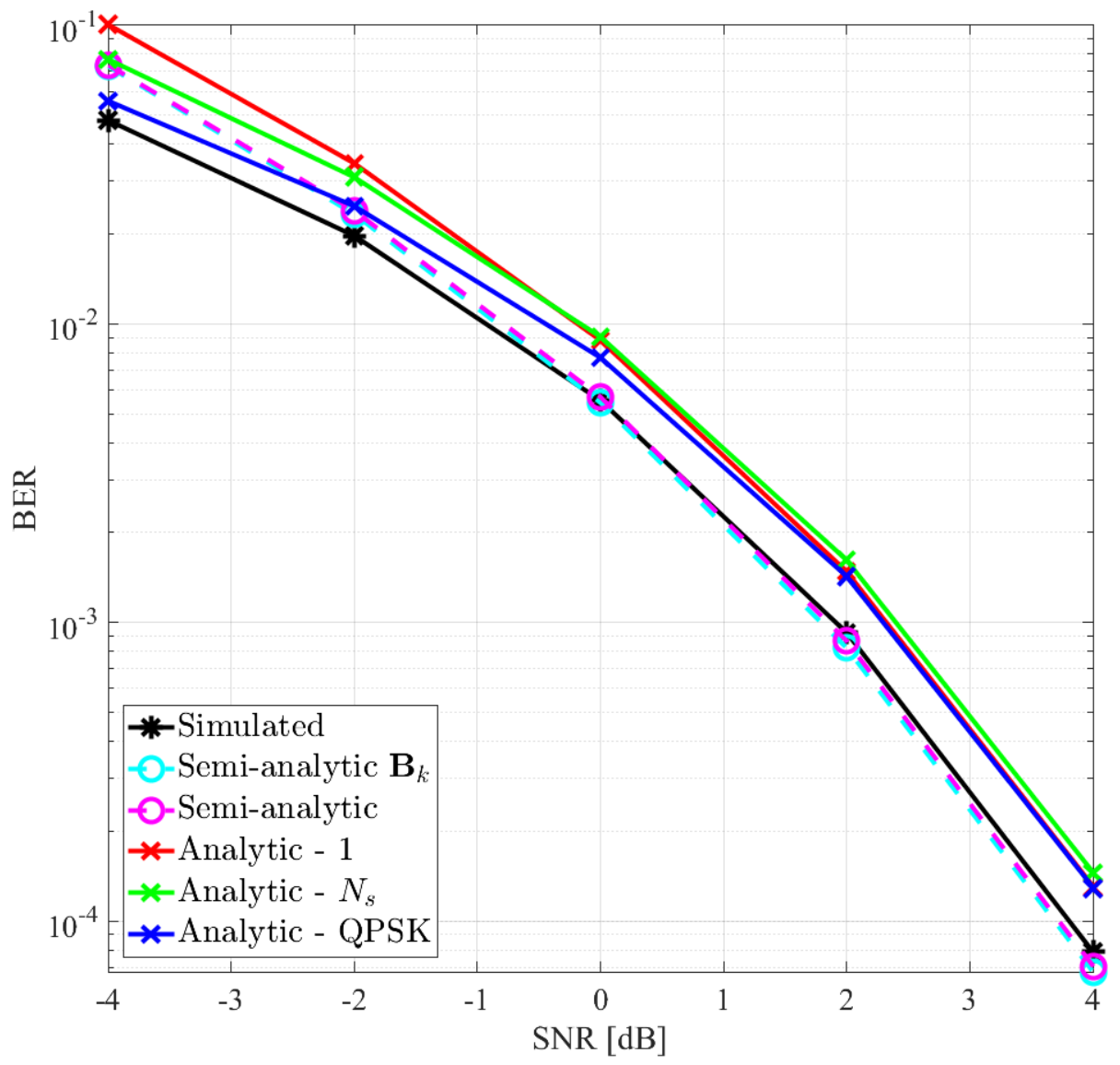

Figure 5.8: Simulated and estimated values of the BER performance using PSVD*-MMSE* ${ }^{*}$. Simulation settings: MDD, $N_{t}=64, N_{s}=2, K=4, N_{r}=4$ and $\hat{\mathbf{B}}_{k}=\mathbf{B}_{k}$. 
Chapter 5. Method to find fairly tight analytic approximations to the BER performance

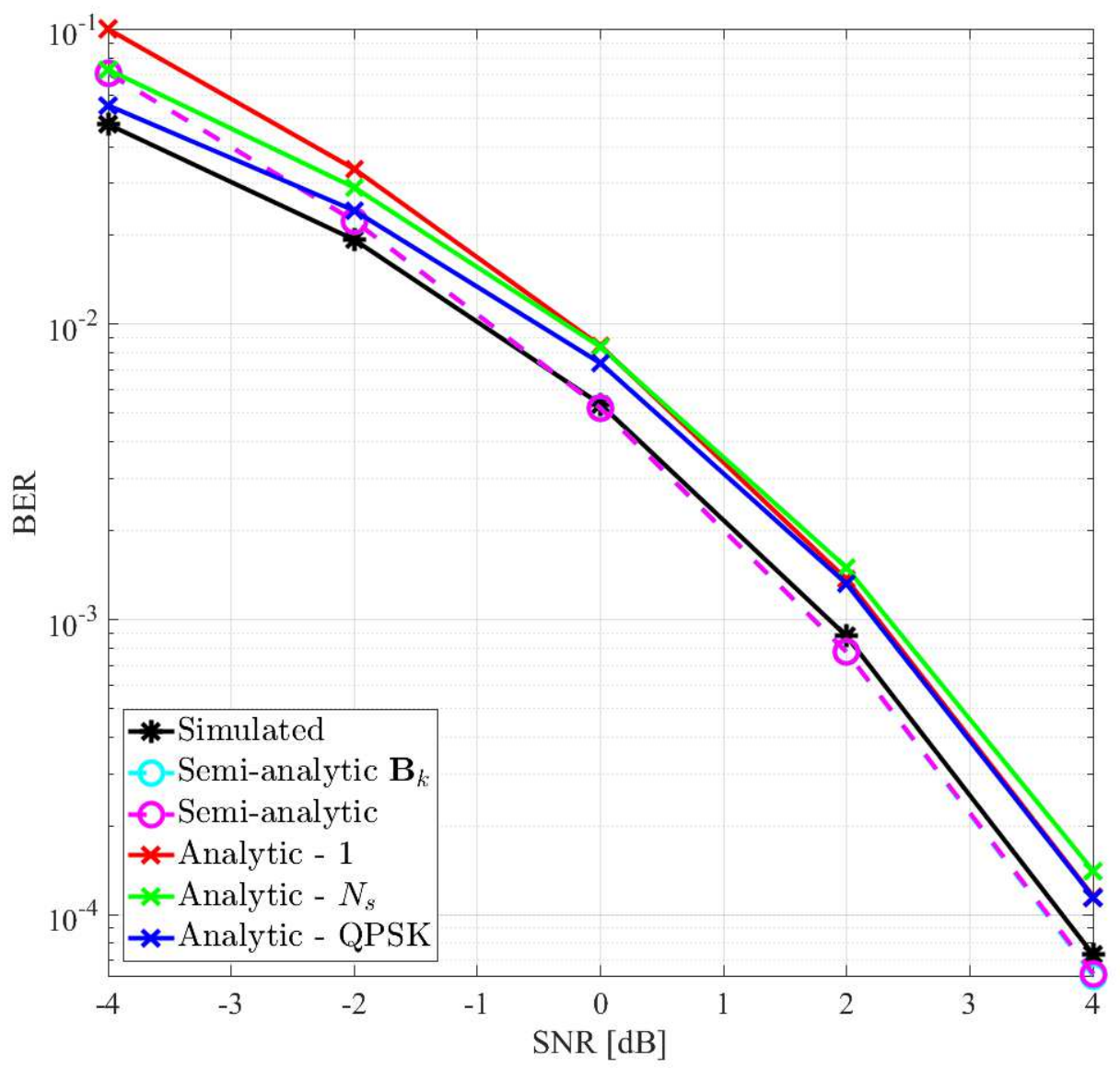

Figure 5.9: Simulated and estimated values of the BER performance using P-SVD*-MMSE*, Simulation settings: NWMDD, $N_{t}=64, N_{s}=2, K=4$, $N_{r}=4$ and $\hat{\mathbf{B}}_{k}=\mathbf{B}_{k}$. 
Chapter 5. Method to find fairly tight analytic approximations to the BER performance

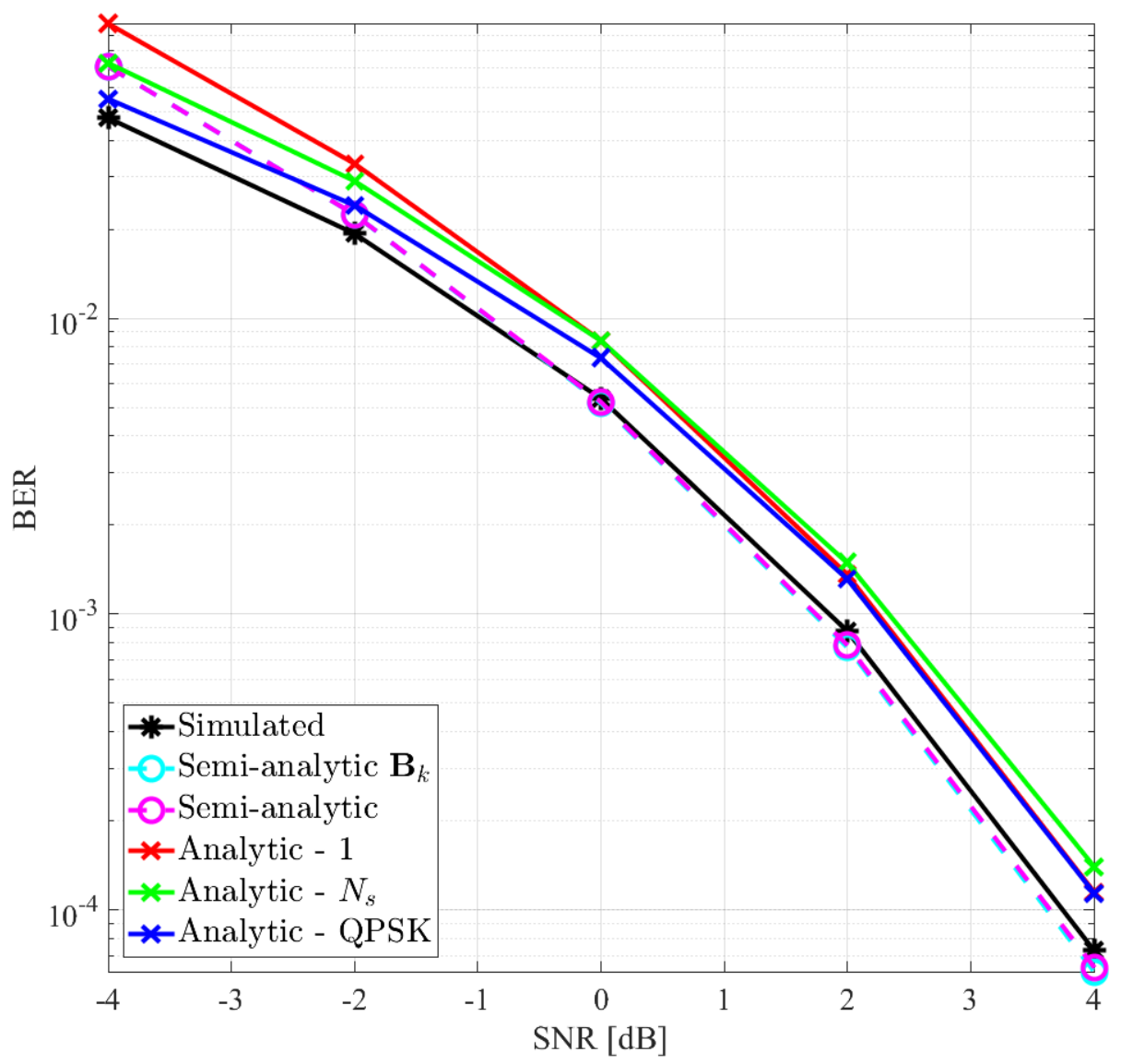

Figure 5.10: Simulated and estimated values of the BER performance using P-SVD*-MMSE*,. Simulation settings: NIWMDD, $N_{t}=64, N_{s}=2, K=4$, $N_{r}=4$ and $\hat{\mathbf{B}}_{k}=\mathbf{B}_{k}$. 
Chapter 5. Method to find fairly tight analytic approximations to the BER performance

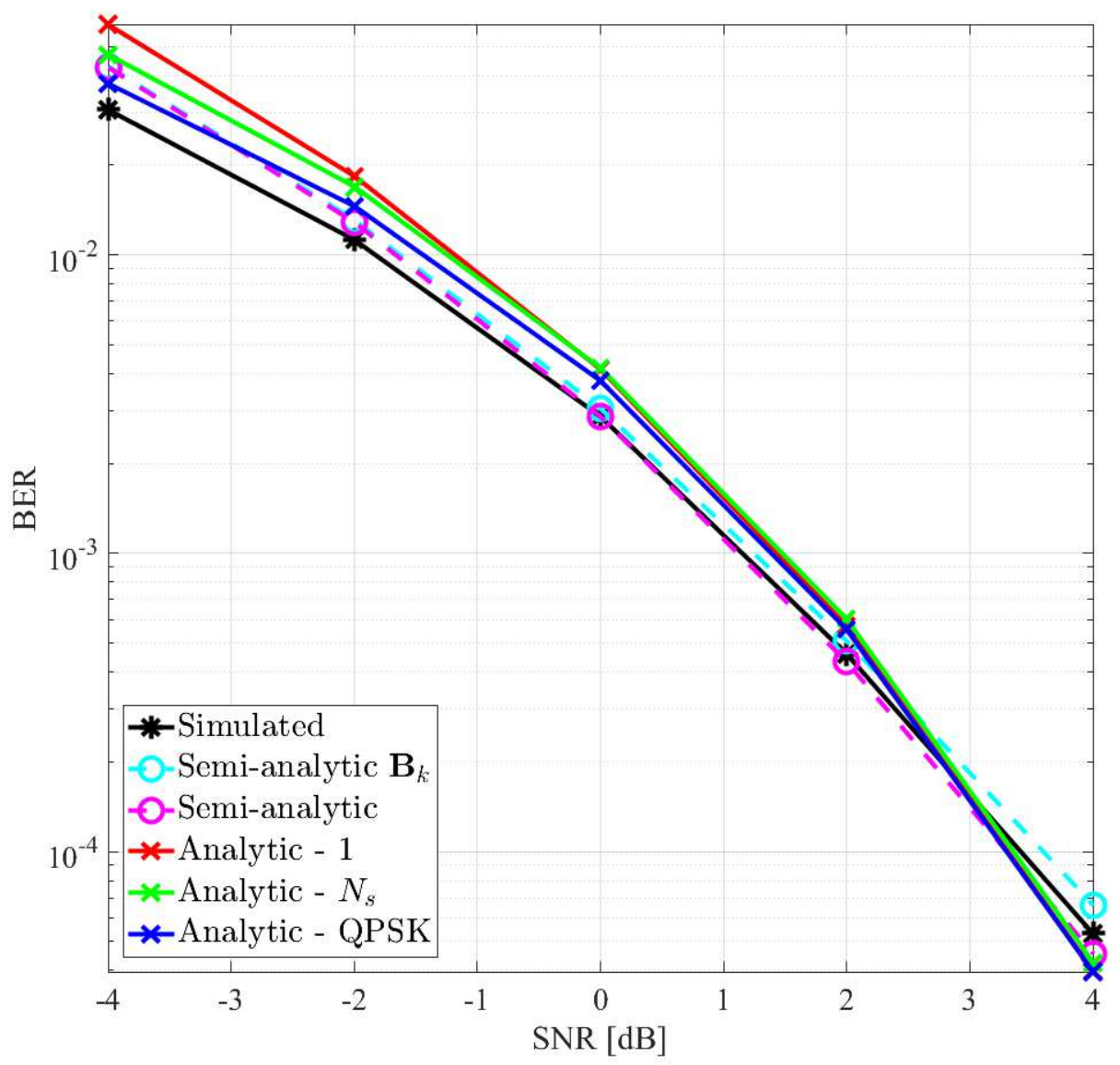

Figure 5.11: Simulated and estimated values of the BER performance using PSVD $^{*}$-MMSE* ${ }^{*}$. Simulation settings: MDD, $N_{t}=64, N_{s}=2, K=8, N_{r}=16$ and $\hat{\mathbf{B}}_{k}=\mathbf{B}_{k}$. 
Chapter 5. Method to find fairly tight analytic approximations to the BER performance

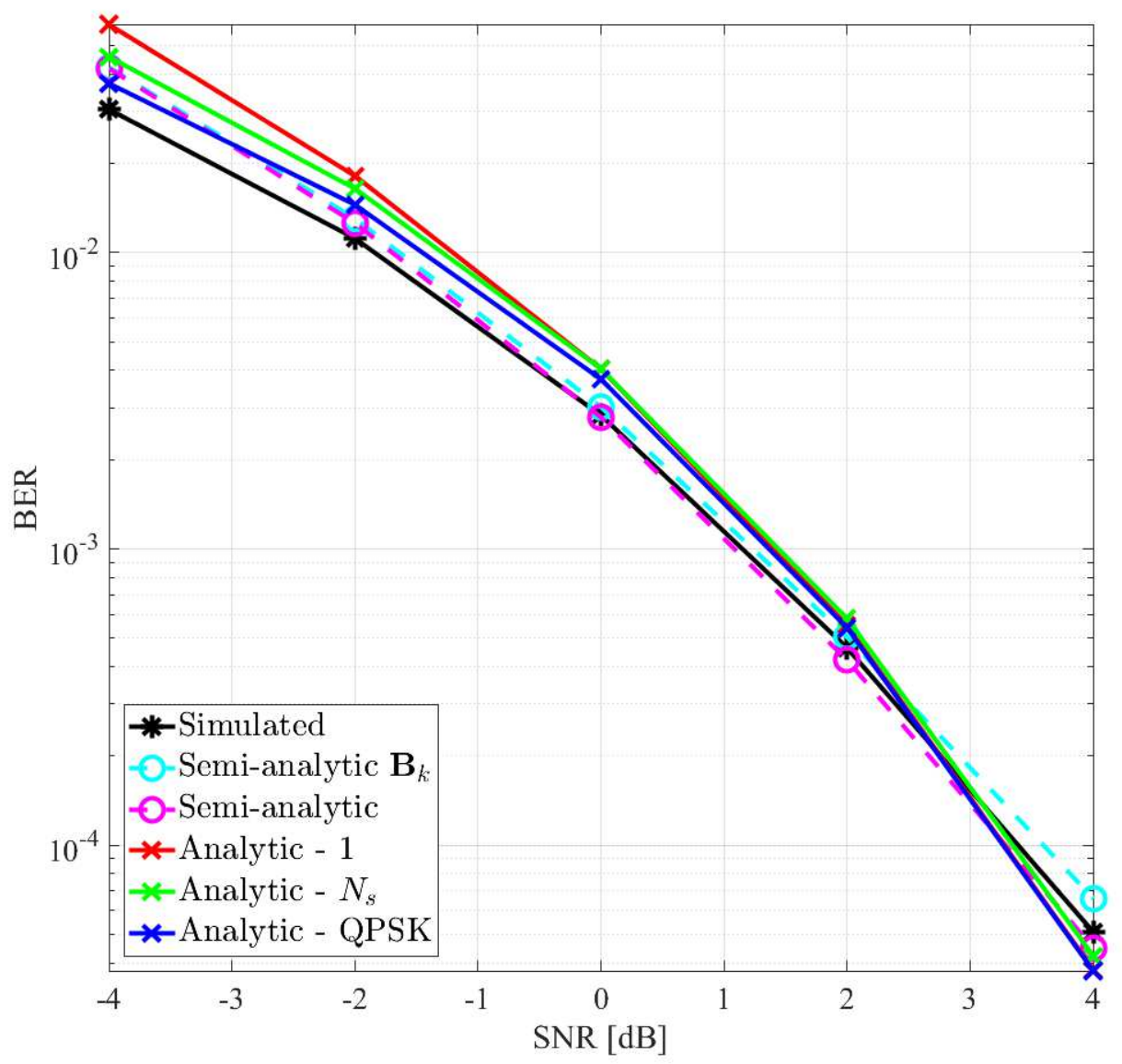

Figure 5.12: Simulated and estimated values of the BER performance using P-SVD*-MMSE*,. Simulation settings: NWMDD, $N_{t}=64, N_{s}=2, K=8$, $N_{r}=16$ and $\hat{\mathbf{B}}_{k}=\mathbf{B}_{k}$. 
Chapter 5. Method to find fairly tight analytic approximations to the BER performance

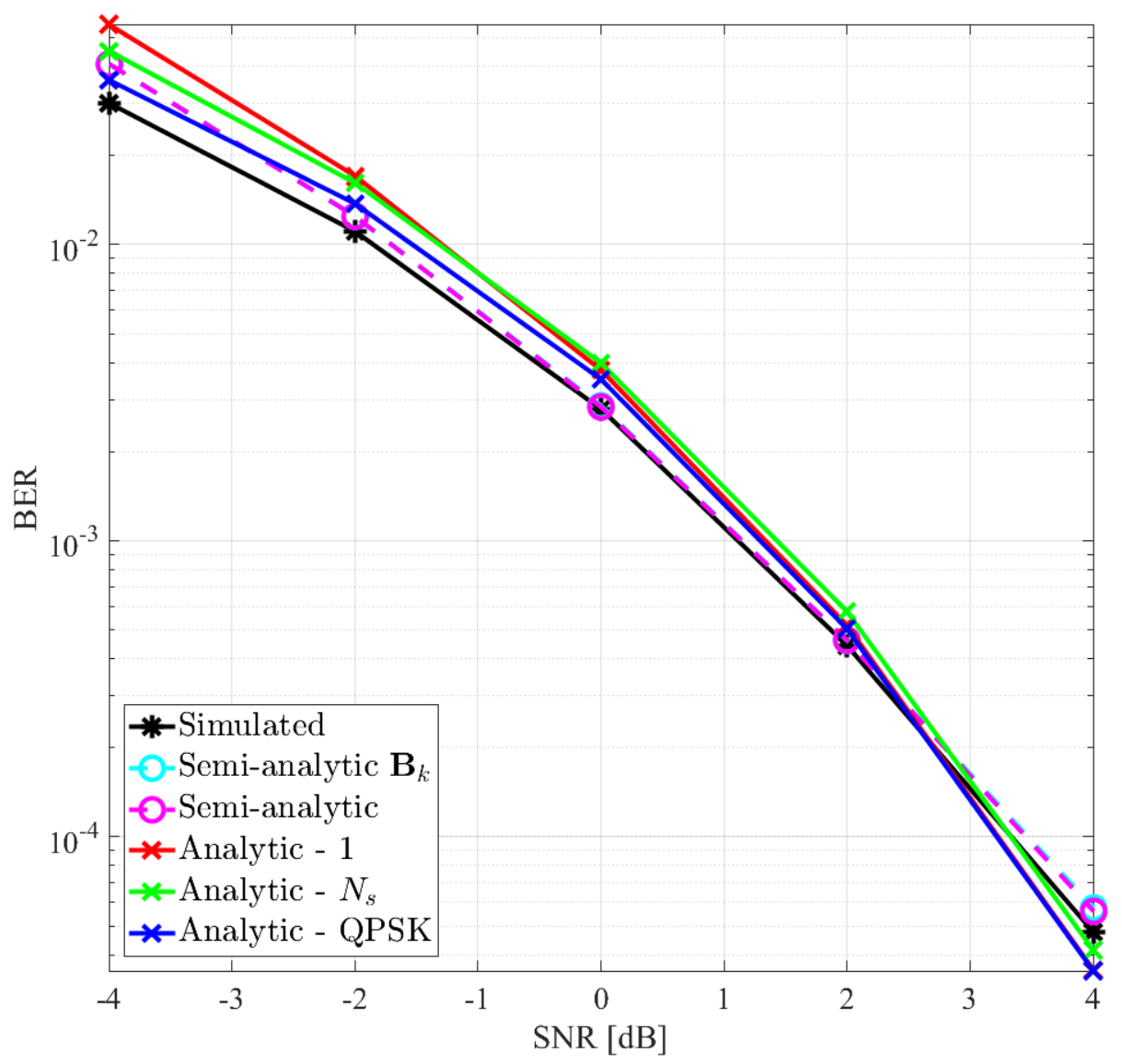

Figure 5.13: Simulated and estimated values of the BER performance using P-SVD*-MMSE*,. Simulation settings: NIWMDD, $N_{t}=64, N_{s}=2, K=8$, $N_{r}=16$ and $\hat{\mathbf{B}}_{k}=\mathbf{B}_{k}$. 
Chapter 5. Method to find fairly tight analytic approximations to the BER performance

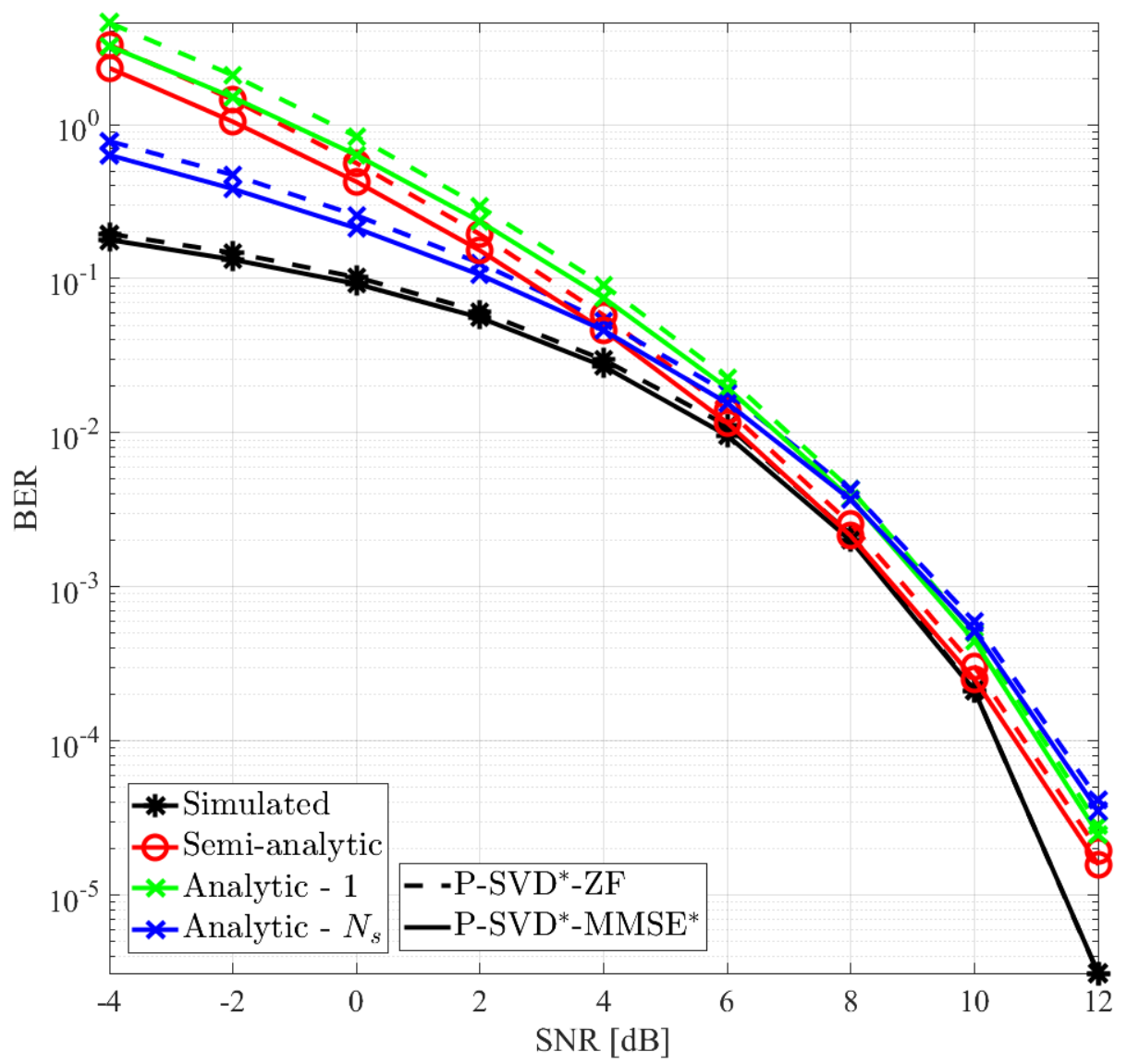

Figure 5.14: BER performance of P-SVD*-ZF and P-SVD*-MMSE* using NWMDD. Simulation settings: $N_{t}=64, K=4, N_{r}=4, N_{s}=2$, and modulation 16QAM 


\section{6}

\section{Three stages hybrid precoder proposal}

Hybrid precoders, which combine large dimensional analog processing with lower dimensional digital processing, are the most promising approach for reducing the hardware cost in massive mmWave MU-MIMO systems and enabling the next-generation wireless communication. This chapter proposes a novel hybrid precoder design made by three stages, where the first stage performs the analog part of the beamforming that maximizes the effective channel gain, while the second and third states perform the digital part of the precoder. The second stage applies a low-dimensional baseband precoder to manage the inter user/symbol interference and the third stage allocates power to decrease the instantaneous BER values. Numerical results show a superior BER performance of our proposal when compared to other hybrid precoders proposed recently.

The remainder of this chapter is organized as follows: Section 6.1 is dedicated to describe our proposal, and in Section 6.2 numerical results and some observations are presented.

\section{1}

\section{Hybrid precoder proposal}

The hybrid beamforming is divided in two parts, the analog part and digital part as stated before. Our proposal to design these parts reflects the idea that the analog part should lead to an equivalent baseband user channel close to a diagonal matrix with large entries values, while the digital part performs a low baseband linear precoder reducing the inter user/symbol interference.

In order to minimize the BER of the system, we consider the design of the digital part of the hybrid precoder in two stages, where the first stage implements a low baseband linear precoder and the second stage effects an algorithm to allocate the available transmit power. Thus, the digital part of the precoder is written as follows

$$
\mathbf{F}_{B B}=\mathbf{F}_{B B_{1}} \mathbf{F}_{B B_{2}}
$$

where $\mathbf{F}_{B B_{2}}=\operatorname{blkdiag}\left(\boldsymbol{\Phi}_{1}, \ldots, \boldsymbol{\Phi}_{K}\right)$, with $\boldsymbol{\Phi}_{K}=\operatorname{diag}\left(\phi_{1, k}, \ldots, \phi_{N_{s}, k}\right), \phi_{l, k} \in$ $\mathbb{R}^{+}, \forall l, k$ and $\operatorname{tr}\left\{\mathbf{F}_{B B_{2}}\right\}=E_{T}$. 
This section is organized as follows: Subsection 6.1.1 presents the main steps for generating the matrices $\mathbf{W}_{R F_{k}}, \mathbf{W}_{B B_{k}}, \mathbf{F}_{R F}$ and $\mathbf{F}_{B B_{1}}$ according to Chapter 4 and 5, which have shown a superior BER performance when compared with different hybrid designs described in the literature; and Subsection 6.1.2 is dedicated to explain our proposal for the generation of the allocated power matrix.

\subsection{1}

\section{Hybrid design review}

Algorithm 10 and 11 resume the steps to generate the hybrid combiner and hybrid precoder, respectively, according to Chapters 4 and 5 .
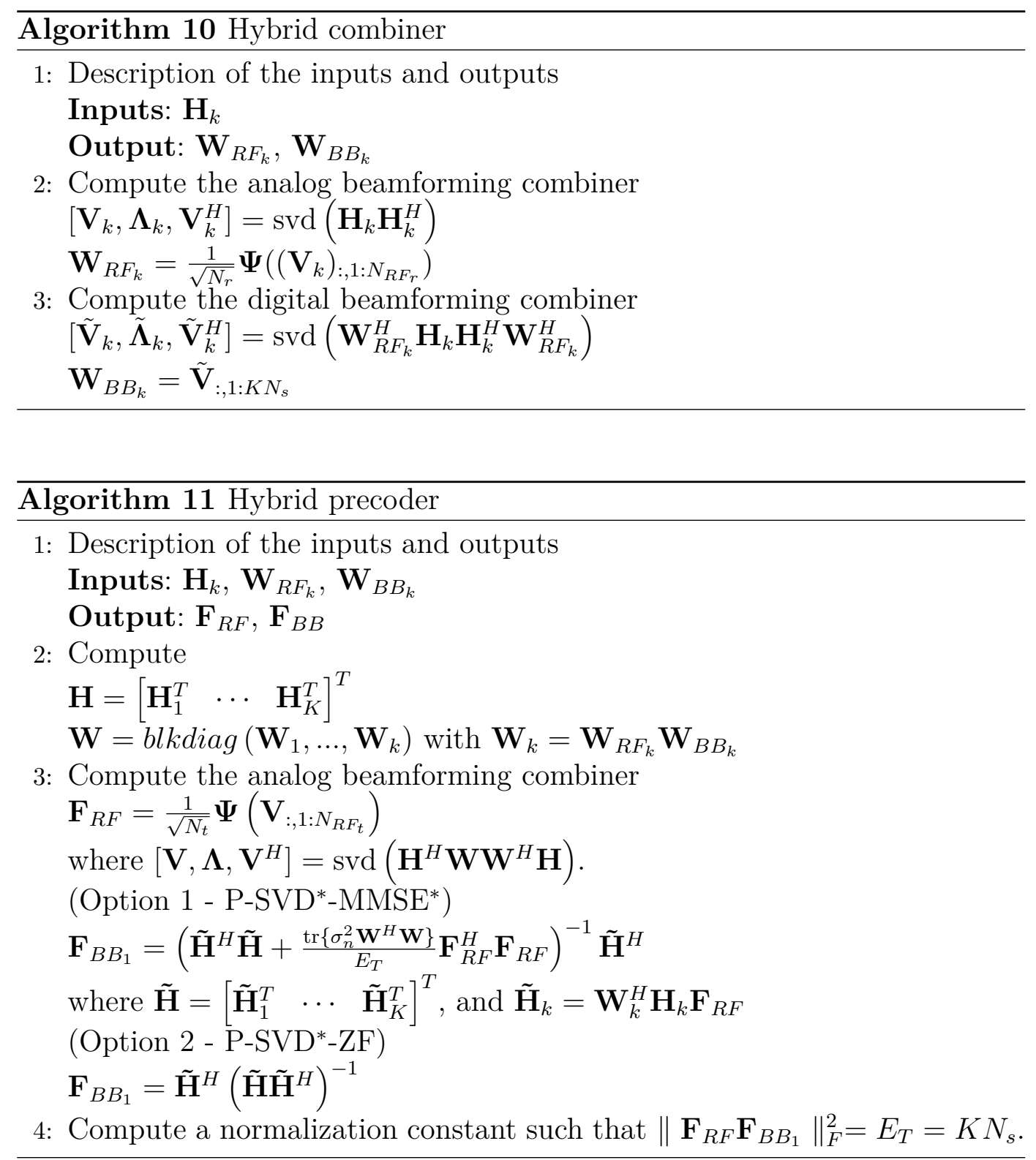


\subsection{2}

\section{Power allocation matrix proposal}

Many power allocating matrices have been proposed in the literature. The majority of them look for the sum rate maximization of the system. However, few works are focused on decreasing the BER performance. Our proposal applies a methodology similar to the one presented in [52] to our considered problem described below.

Considering (6-1) the processed received signal given by (2-2) can be written as

$$
\mathbf{y}_{k}=\mathbf{W}_{k}^{H} \mathbf{H}_{k} \mathbf{F}_{k} \boldsymbol{\Phi}_{k} \mathbf{s}_{k}+\sum_{j \neq k} \mathbf{W}_{k}^{H} \mathbf{H}_{k} \mathbf{F}_{j} \boldsymbol{\Phi}_{j} \mathbf{s}_{j}+\mathbf{W}_{k}^{H} \mathbf{n}_{k}
$$

where $\left[\begin{array}{lll}\mathbf{F}_{1} & \cdots & \mathbf{F}_{K}\end{array}\right]=\mathbf{F}_{R F} \mathbf{F}_{B B_{1}}$. The first (second) term correspond to the wanted (interference) signal, and the third term represents the noise.

The expression (6-2) can be rewritten as

$$
\mathbf{y}_{k}=\mathbf{W}_{k}^{H} \mathbf{H}_{k} \mathbf{F}_{k} \boldsymbol{\Phi}_{k} \mathbf{s}_{k}+\tilde{\mathbf{n}}_{k}
$$

where the equivalent noise $\tilde{\mathbf{n}}_{k}=\sum_{j \neq k} \mathbf{W}_{k}^{H} \mathbf{H}_{k} \mathbf{F}_{j} \boldsymbol{\Phi}_{j} \mathbf{s}_{j}+\mathbf{W}_{k}^{H} \mathbf{n}_{k}$ has covariance $\operatorname{matrix} \mathbf{K}_{k}=\mathbb{E}\left[\tilde{\mathbf{n}}_{k} \tilde{\mathbf{n}}_{k}^{H}\right]=\sigma_{n}^{2} \mathbf{W}_{k}^{H} \mathbf{W}_{k}+\mathbf{K}_{\mathbf{i}_{\mathbf{k}}}$, with $\mathbf{K}_{\mathbf{i}_{\mathbf{k}}}=\mathbb{E}\left[\mathbf{i}_{k} \mathbf{i}_{k}^{H}\right]$, and $\mathbf{i}_{k}=$ $\sum_{j \neq k} \mathbf{W}_{k}^{H} \mathbf{H}_{k} \mathbf{F}_{j} \boldsymbol{\Phi}_{j} \mathbf{s}_{j}$. If the interference term is a Gaussian vector ${ }^{1}$ statistically independent of the noise vector, then, $\tilde{\mathbf{n}}_{k} \sim \mathcal{C N}\left(\mathbf{0}, \mathbf{K}_{k}\right)$ and the ML estimate of the received data can be obtained by

$$
\hat{\mathbf{s}}_{k}=\arg \min _{\mathbf{m} \in \mathbb{Q}^{N_{s}}}\left\|\mathbf{A}_{k}^{-1 / 2}\left(\mathbf{y}_{k}-\mathbf{B}_{k} \boldsymbol{\Phi}_{k} \mathbf{m}\right)\right\|^{2}
$$

where $\mathbf{B}_{k}=\mathbf{W}_{B B_{k}}^{H} \mathbf{W}_{R F_{k}}^{H} \mathbf{H}_{k} \mathbf{F}_{k}$, and $\mathbf{A}_{k}=\mathbf{K}_{k}$. Considering (6-4) it can be shown that given $\mathbf{H}_{k}$ and $\boldsymbol{\Phi}_{k},(k=1, \ldots, K)$, the pairwise error probability based upper bound of the conditional BER of the system is

$$
\mathrm{CBER} \leq c \sum_{k=1}^{K} \sum_{i=1}^{M} \sum_{j \neq 1}^{M} d_{b_{i j}} Q\left(\sqrt{\frac{\mathbf{d}_{i j}^{H} \boldsymbol{\Phi}_{k}^{H} \mathbf{B}_{k}^{H} \mathbf{K}_{k}^{-1} \mathbf{B}_{k} \boldsymbol{\Phi}_{k} \mathbf{d}_{i j}}{2}}\right)
$$

where $c=\left(K M \log _{2}(M)\right)^{-1} ; M$ is the number of possible transmitted symbol vectors for the user $k ; d_{b_{i j}}$ is the Hamming distance between the equivalent bit representation of the symbol vectors $\mathbf{s}_{i} ; \mathbf{s}_{j} ; \mathbf{d}_{i j}=\mathbf{s}_{i}-\mathbf{s}_{j}$; and $\mathbf{s}_{i} \in \mathbb{Q}^{N_{s}} \forall i$.

Considering $\mathbf{K}_{i_{k}} \approx \tilde{\mathbf{K}}_{i_{k}}=\mathbb{E}\left[\tilde{\mathbf{i}}_{k} \tilde{\mathbf{i}}_{k}^{H}\right]$, with $\tilde{\mathbf{i}}_{k}=\sum_{j \neq k} \mathbf{W}_{k}^{H} \mathbf{H}_{k} \mathbf{F}_{j} \mathbf{s}_{j}$, then $\mathbf{K}_{k} \approx \tilde{\mathbf{K}}_{k}=\sigma_{n}^{2} \mathbf{W}_{k}^{H} \mathbf{W}_{k}+\tilde{\mathbf{K}}_{\mathbf{i}_{\mathbf{k}}}$ and therefore, the product $\mathbf{B}_{k}^{H} \mathbf{K}_{k}^{-1} \mathbf{B}_{k} \approx$ $\mathbf{B}_{k}^{H} \tilde{\mathbf{K}}_{k}^{-1} \mathbf{B}_{k}$. If this product is a diagonal matrix, then the system has no inter-

\footnotetext{
${ }^{1}$ In fact the interference term can be modeled as a Gaussian vector in a pessimistic case, i.e., large number of users, through the central limit theorem.
} 
symbol interference, and the detection in (6-4) can be made symbol by symbol separately, and the upper bound (6-5) can be written as

$$
\mathrm{CBER} \leq \tilde{c} \sum_{k=1}^{K} \sum_{l=1}^{N_{s}} \sum_{i=1}^{N} \sum_{j \neq 1}^{N} \tilde{d}_{b_{i j}} Q\left(\sqrt{\frac{\gamma_{l}^{(k)}\left\|\tilde{d}_{i j}\right\|^{2} \phi_{l, k}^{2}}{2}}\right)
$$

where $\left(\gamma_{1}^{(k)}, \ldots, \gamma_{N_{s}}^{(k)}\right)=\operatorname{diag}\left(\mathbf{B}_{k}^{H} \tilde{\mathbf{K}}_{k}^{-1} \mathbf{B}_{k}\right) ; \tilde{c}=\left(K N N_{s} \log _{2}(N)\right)^{-1} ; N$ is the size of the signal constellation $\mathbb{Q}$; $\tilde{d}_{b_{i j}}$ is the Hamming distance between the equivalent bit representation of the symbols of the constellation $s_{i}$ and $s_{j}$; $\tilde{d}_{i j}=s_{i}-s_{j}$; and $s_{i} \in \mathbb{Q}, \forall i$.

If the QPSK or BPSK modulation is used, an approximation to the conditional BER of the system can be obtained through

$$
\mathrm{CBER} \approx \frac{1}{K N_{s}} \sum_{k=1}^{K} \sum_{l=1}^{N_{s}} Q\left(\sqrt{\gamma_{l}^{(k)} \phi_{l, k}^{2}}\right)
$$

Thus, we consider the following problem

$$
\begin{array}{r}
\min _{\phi_{l, k}} \frac{1}{K N_{s}} \sum_{k=1}^{K} \sum_{l=1}^{N_{s}} Q\left(\sqrt{\gamma_{l}^{(k)} \phi_{l, k}^{2}}\right) \\
\text { s.t. } \sum_{k=1}^{K} \sum_{l=1}^{N_{s}} \phi_{l, k}^{2}=E_{T}
\end{array}
$$

Considering the fact that the function $Q(\sqrt{x}) \approx \alpha e^{-\beta x}$, with $\alpha=\beta=0.5$, and making use of the Langrange method, the cost function of (6-8) can be written as

$$
\xi=\frac{1}{K} \sum_{k=1}^{K} \sum_{l=1}^{N_{s}} \alpha e^{-\beta \tilde{\gamma}_{l}^{(k)} \phi_{l, k}^{2}}+\mu\left(\sum_{k=1}^{K} \sum_{l=1}^{N_{s}} \phi_{l, k}^{2}-E_{T}\right)
$$

where $\mu$ represents the Lagrange multiplier. Taking the derivative of (6-9) with respect to $\phi_{l, k}$

$$
\frac{\partial \xi}{\partial \phi_{l, k}}=\frac{-2 \alpha \beta \gamma_{l}^{(k)} \phi_{l, k}}{K} e^{-\frac{1}{\tilde{\sigma}_{k}^{2}} \beta \gamma_{l}(k) \phi_{l, k}^{2}}+2 \mu \phi_{l, k}
$$

and equating (6-10) to zero yields

$$
\phi_{l, k}^{2}=\frac{\ln \gamma_{l}^{(k)}-\mu}{\beta \gamma_{l}{ }^{(k)}}
$$

Substituting (6-11) in the power constraint in (6-8) yields

$$
\mu=\frac{\sum_{k=1}^{K} \sum_{l=1}^{N_{s}} \frac{\ln \gamma_{l}^{(k)}}{\beta \gamma_{l}^{(k)}}-E_{T}}{\sum_{k=1}^{K} \sum_{l=1}^{N_{s}} \frac{1}{\beta \gamma_{l}^{(k)}}}
$$

Note that the above solution does not take into account the inherent inequality constraints, $\phi_{l, k}^{2} \geq 0, \forall l, k$. Therefore, when one or more of these 
constraints are not satisfied, the Lagrange multiplier should be found via waterfilling algorithm. Algorithm 12 presents an option to implement the waterfilling algorithm for a general case [40]. In order to apply Algorithm 12 to our particular problem (6-11), we introduce a restriction in (6-11) by defining

$$
\phi_{l, k}^{2}=\left(\frac{\mu-\ln \frac{1}{\gamma_{l}^{(k)}}}{\beta \gamma_{l}^{(k)}}\right)_{+}
$$

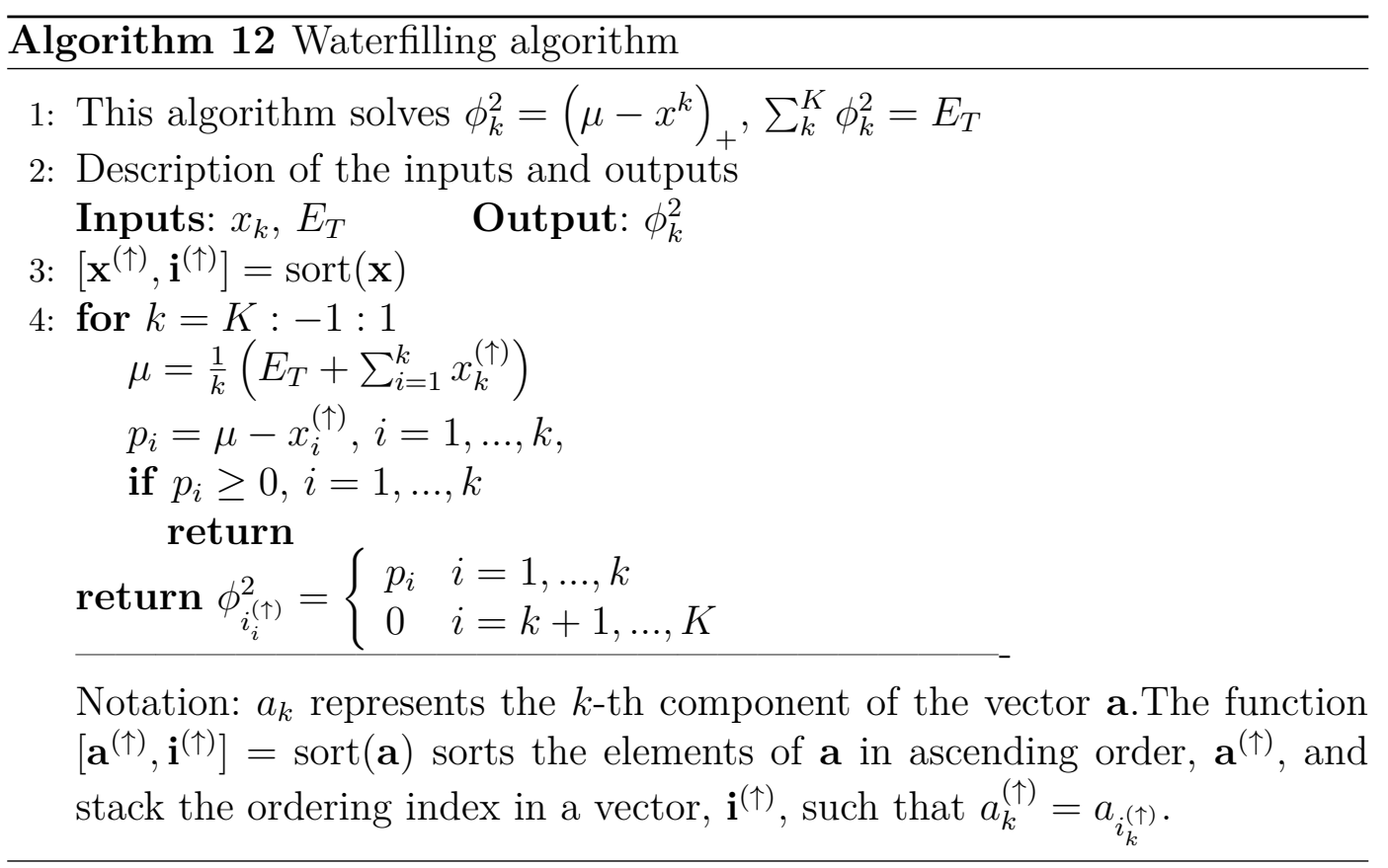

Finally, in order to satisfy the power constraint on the whole hybrid precoder, a normalization constant have to be computed such that $\left\|\mathbf{F}_{R F} \mathbf{F}_{B B_{1}} \mathbf{F}_{B B_{2}}\right\|_{F}^{2}=K N_{s}$. Note that two options for $\mathbf{F}_{B B_{1}}$ were presented in Algorithm 11, therefore to distinguish the different resulted hybrid precoders, the following notation is used: P-SVD*-S ${ }_{1}+\mathrm{PA}$, where $\mathrm{S}_{1} \in\left\{\mathrm{MMSE}^{*}, \mathrm{ZF}\right\}$.

\section{2}

\section{Numerical results}

In the simulations, the users' channels are generated with $N_{p}=10$ multi-path components, the azimuth and elevation departure angles values are given by a random variable with uniform distribution in the interval of $(0,2 \pi)$ and $(0, \pi)$, respectively. The UPAs have square formats for both transmitter and receivers, i.e., $N_{t_{h}}=N_{t_{v}}=\sqrt{N}_{t}$ and $N_{r_{h}}=N_{r_{v}}=\sqrt{N}_{r}$. The minimum allowed setting for the number of RF chains is used for both the BS and for each MS, so that $N_{R F_{t}}=K N_{s}$ and $N_{R F_{r}}=N s$. The results are averaged over $8 \times 10^{5}$ channel realizations for each user. In addition, the three data detection approaches presented in Chapter 4 was considered: Minimum 
distance detection (MDD) : $\mathbf{A}_{k}=\mathbf{I}_{N_{s}}$ in (6-4); Noise whitening operation followed by MDD (NWMDD) : $\mathbf{A}_{k}=\sigma_{n}^{2} \mathbf{W}_{k}^{H} \mathbf{W}_{k}$ in (6-4); and Noise and interference whitening operation followed by MDD (NIWMDD) : $\mathbf{A}_{k}=\mathbf{K}_{k}$ in $(6-4)$.

For Figures 6.1 and 6.2 the simulated hybrid system has the following characteristics: the BS has $N_{t}=64$ antennas and sends $N_{s}=2$ streams using modulation QPSK to $K=4$ users equipped with $N_{r}=4$.

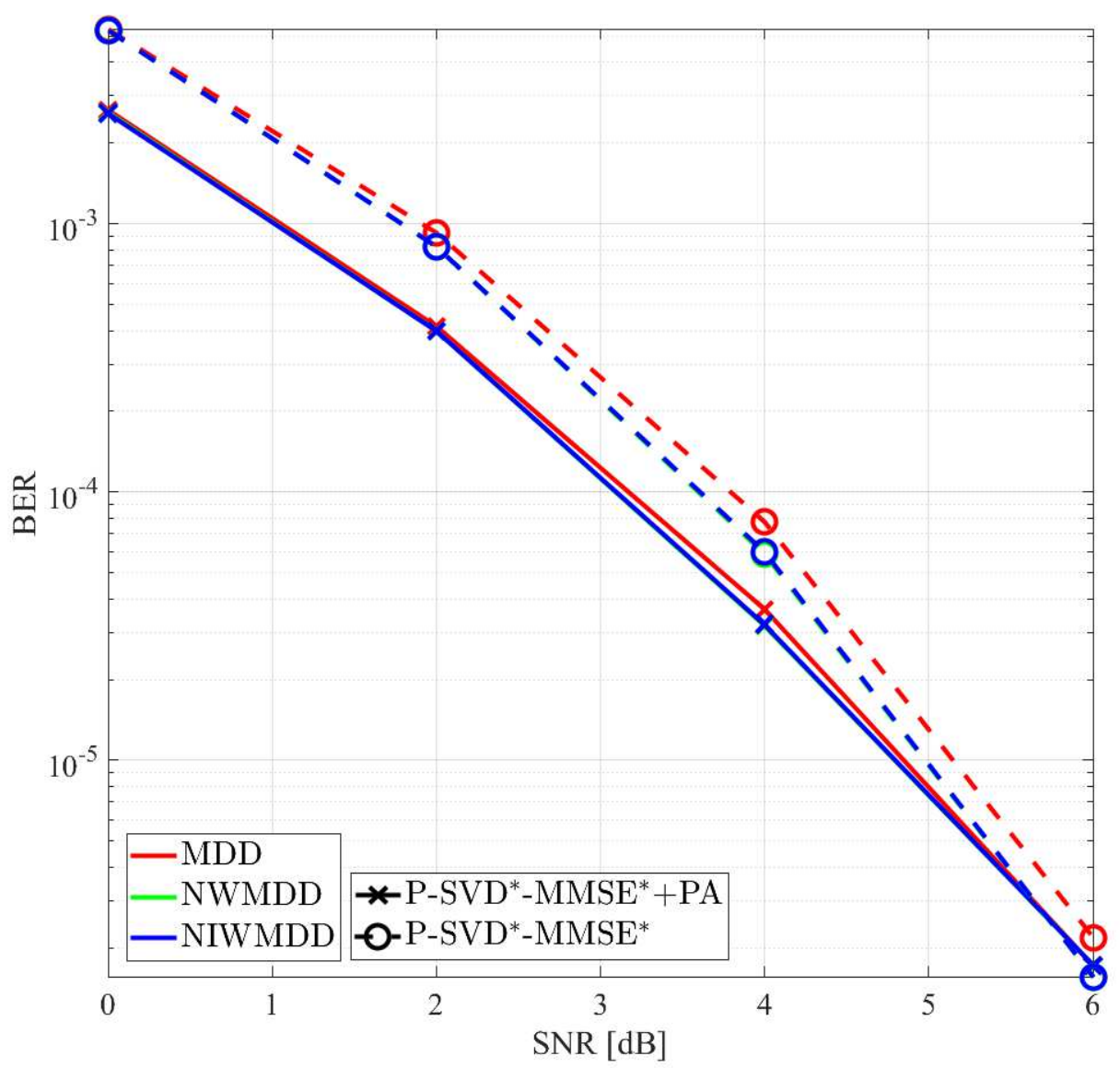

Figure 6.1: BER performance comparison between our proposals P-SVD*MMSE* $^{*}+\mathrm{PA}$ and P-SVD ${ }^{*}$-MMSE* $. N t=64, N_{r}=4, N_{s}=2$ and $K=4$

For Figures 6.3 and 6.4 the simulation settings are: the BS has $N_{t}=64$ antennas and sends $N_{s}=2$ streams using modulation QPSK to $K=8$ users equipped with $N_{r}=16$.

Figures 6.1 - 6.4 show that the addition of the proposed power allocating matrix to the considered hybrid precoders gives a improvement in the BER performance, which satisfies the target of the methodology proposed in this chapter. Figures 6.1 and 6.2 evidenced the greatest improvements, which means that proposed power allocating matrix is more efficient when the number of users is low. 


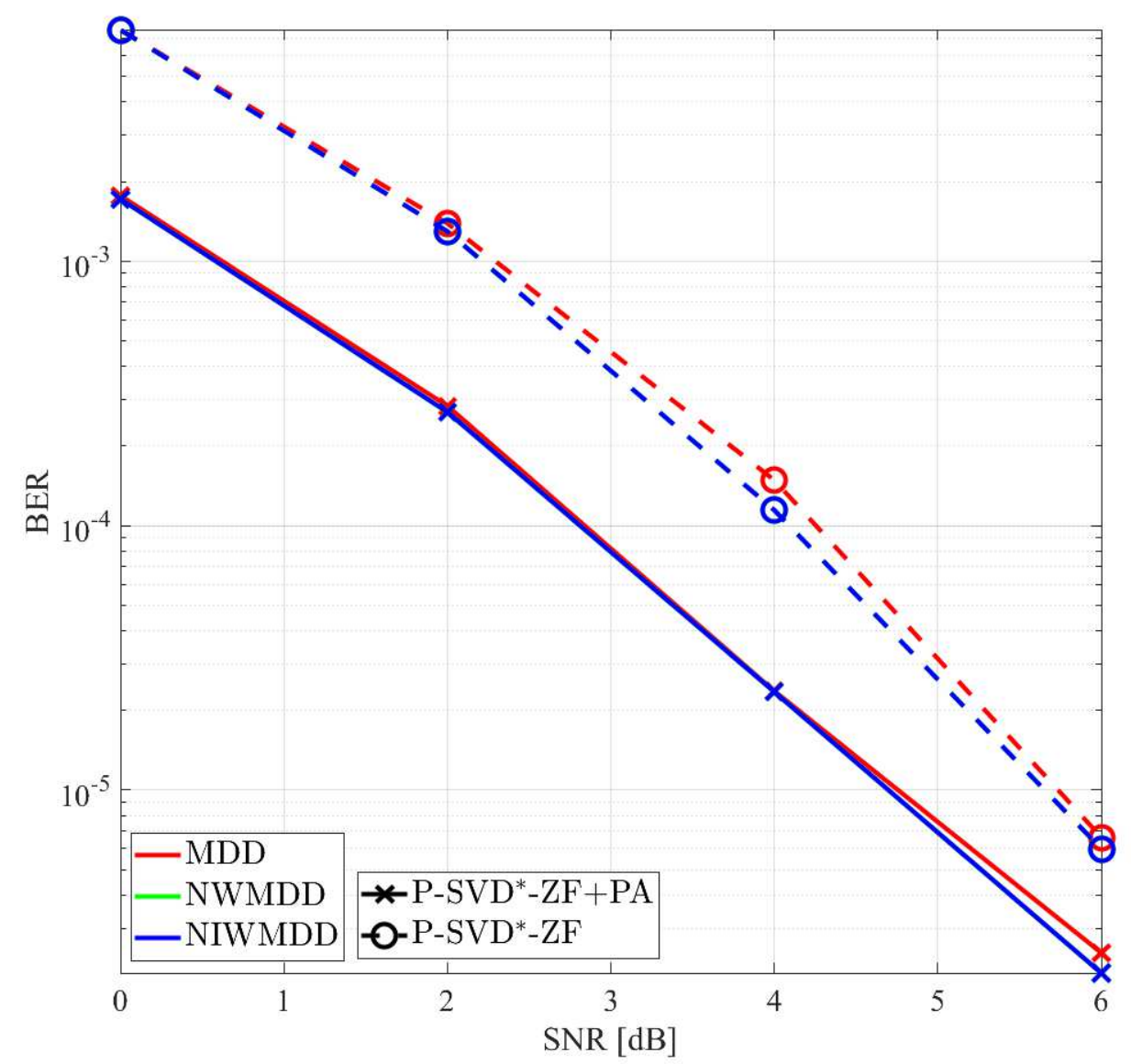

Figure 6.2: BER performance comparison between our proposal P-SVD*$\mathrm{ZF}+\mathrm{PA}$ and P-SVD ${ }^{*} \mathrm{ZF} . N t=64, N_{r}=4, N_{s}=2$ and $K=4$

Table 6.1 presents a counter of the number of times that the water filling was used to compute the lagrange multiplier over the $8 \times 10^{5}$ channel realizations.

The values in Table 6.1 evidence that for $\mathrm{P}_{-S V D}$-MMSE*$+\mathrm{PA}$ the Lagrange multiplier value was found through (6-12) in the large majority of cases. However, it is observed that when the number of users increases and the SNR value decreases, this counter tends to increase. In contrast, P-SVD*$\mathrm{ZF}+\mathrm{PA}$ did not require the water filling algorithm in all considered simulation settings. 


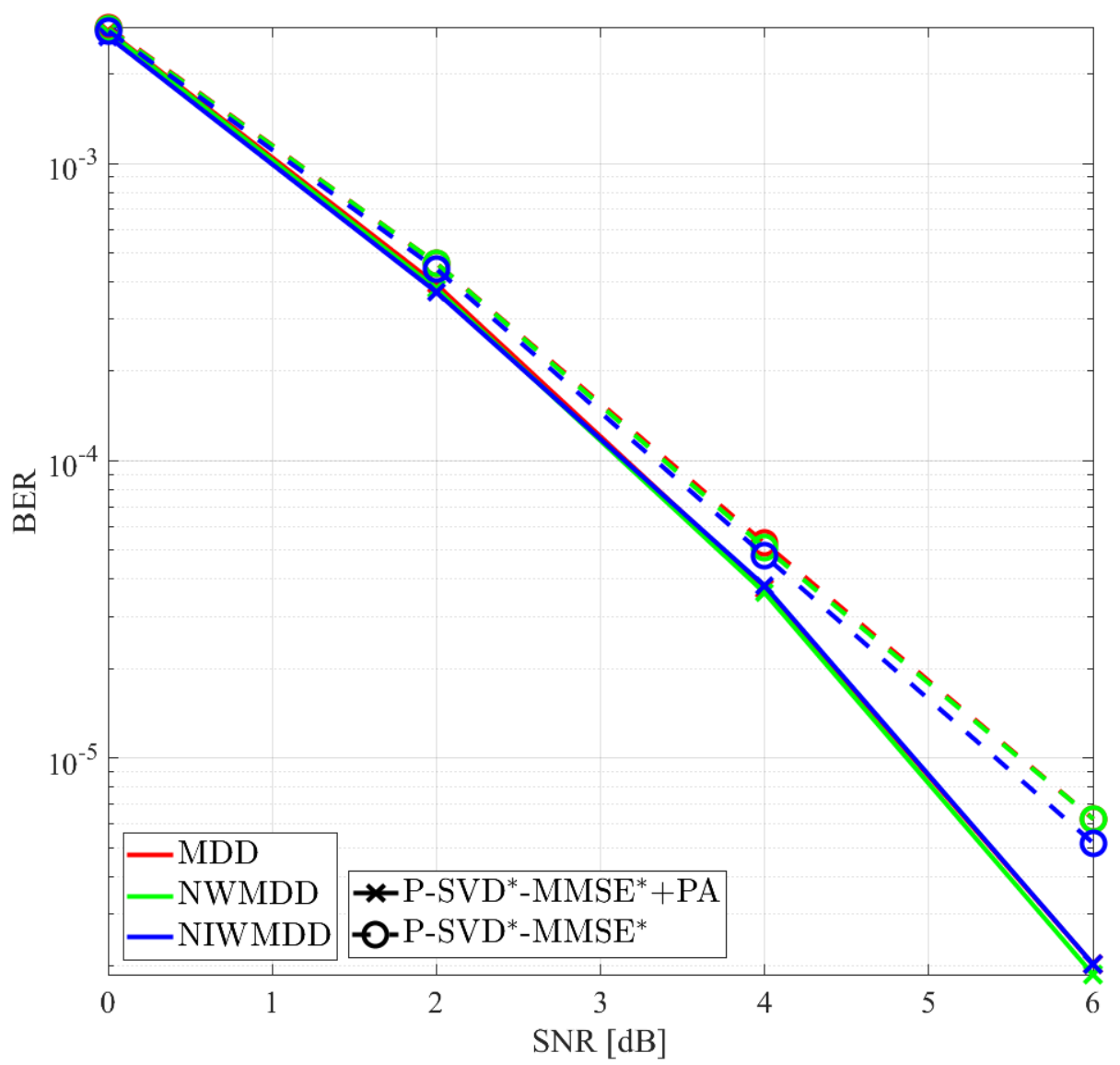

Figure 6.3: BER performance comparison between our proposals P-SVD*MMSE $^{*}+\mathrm{PA}$ and P-SVD*-MMSE ${ }^{*} . N t=64, N_{r}=16, N_{s}=2$ and $K=8$

Table 6.1: Number of times that the water filling algorithm was used

\begin{tabular}{|c|c|c|}
\hline \multirow{2}{*}{ Simulation settings } & \multicolumn{2}{|c|}{ P-SVD*-MMSE* $^{*}+\mathrm{PA}$} \\
\cline { 2 - 3 } & $\mathrm{SNR}=0 \mathrm{~dB}$ & $\mathrm{SNR}=2 \mathrm{~dB}$ \\
\hline$N t=64, N_{r}=4, N_{s}=2$ and $K=4$ & 19 & 2 \\
\hline$N t=64, N_{r}=16, N_{s}=2$ and $K=8$ & 114 & 4 \\
\hline
\end{tabular}




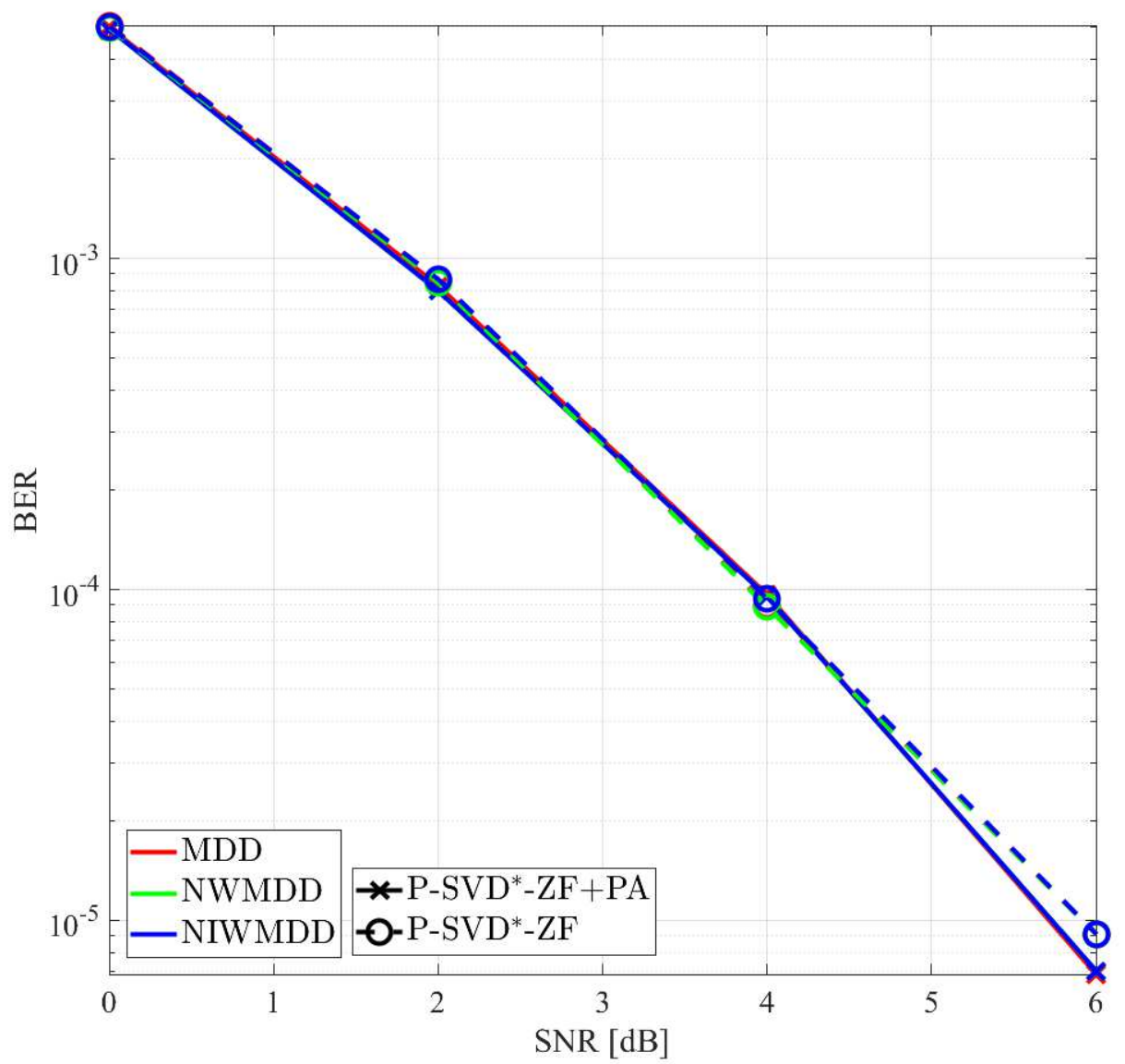

Figure 6.4: BER performance comparison between our proposals P-SVD*$\mathrm{ZF}+\mathrm{PA}$ and P-SVD*-ZF. $N t=64, N_{r}=16, N_{s}=2$ and $K=8$ 


\section{Conclusions}

In Chapter 3, we studied the mutual information rate for downlink massive MU-MIMO mmWave system. Our research was focused on finding an hybrid precoder such that maximizes the mutual information' upper bound. This problem has already studied in mmWave systems for the last years, e.g., $[1,21,23,24,29,32]$, the majority of the proposed solutions try to decrease the distance between the hybrid precoder and a digital one but, that methodology generally leads to iterative complex algorithms. However, the considered problem in this chapter has been widely studied for traditional MIMO system, thus, our research was focused on the adaptation of a traditional solution achieving a good performance. Particularly, Chapter 3 presents the adaptation of [30] to mmwave MU-MIMO scenarios. Numerical results in terms of mutual information rate showed the improvement obtained by our proposal in relation to the other considered hybrid precoders. However, when the BER was used as performance metric, it could be observed that our hybrid precoder proposal did not achieve a good performance due to its low capacity of interference management. Precoder designs through techniques that maximizes the mutual information could lead to solutions also increase the inter user interference. Consequently, the resulted interference in this case could not be mitigate even using an all digital combiner. Therefore, other precoding strategies have to be taking into account to obtain a better BER performance.

Chapter 4 described a new hybrid precoding/combining strategy based on the MMSE linear precoder and the maximization of the mutual information. Using this approach four hybrid combiner/precoder for downlink mmWave massive MU-MIMO systems were proposed. The designing of the hybrid combiner/precoder was divided in two parts, analog and digital. Previous designs of the analog part of the combiner/precoder are focused in the increasing of the SNR, since theses analog parts are not very useful to manage the inter user/symbol interference. In contrast, in our approach, we noticed that the ideal equivalent baseband user channel is a diagonal matrix with large entries, such that our designs try to reach an approximation of this desire result. Numerical results showed that addressing the beamforming phases to a digital precoder (combiner) based on SVD could be a good strategy to construct the 
analog part part of the precoder (combiner). Then, since the digital part of the combiner and precoder do not have constraints for their construction, their designs can be based on traditional signal processing that takes into account the colored noise vector in the received signal.

Furthermore, Chapter 4 presents a exact derivation of the MMSE linear precoder to be used as the digital part. Numerical results illustrate the BER performance improvement obtained through the proposed hybrid designs. In addition, for P-SVD*-MMSE*, which shown the best performance, a simple detection approach can be used to estimate the transmitted data without a significant performance loss.

Chapter 5 derived a general expression to get an upper bound of the BER performance and proposed a method to find fairly tight analytic approximations to the BER performance using different data detectors. Three analytic expressions were derived through our proposed method. Specifically, the presented results shown that when the modulation QPSK or BPSK is used, the most exact and accurate approximation is given by "Analytic - QPSK" for the two considered hybrid precoders and the three data detectors. However, when other kind of modulation is considered, "Analytic - QPSK" should not be used. According to the results using 16QAM, "Analytic - $N_{s}$ " gave very close approximations of the BER performance even better than the BER performance upper bound expression, here referred as "Semi-analytic $\mathbf{B}_{k}$ ".

From the numerical results it could be observed that the entries of the diagonal of $\mathbf{B}_{k}^{H} \mathbf{A}^{-1} \mathbf{B}_{k}$ have a pdf quite similar to the gamma distribution, which facilitated the derivation analytic approximations as functions in terms of the mean and variance of these entries. However, computing these two statistics in an analytic way is mathematically challenging, and therefore, they were obtained via Monte Carlo method.

Finally, in Chapter 6 we proposed a hybrid precoder design made by three stages. The fist stage maximizes the effective channel gain, the second stage applies a low dimensional baseband precoder and the third stage performs an allocated power algorithm to decrease the instantaneous BER performance. The first two stages were took from Chapter 4 due to their superior BER performance when compared to other hybrid precoders from the literature. The results presented in this paper show performance gains when the proposed third stage is added with a low complexity increase of $O\left(K N_{s}\right)$. Particularly, when the number of users is low a better BER performance improvement is obtained especially for the considered hybrid Zero Forcing.

This work can be extended in different manners. Below follows a list of possible options as future works: 
- Considering uplink mmWave MU-MIMO. Note that this whole work is focused on the performance behavior in downlink mmWave MU-MIMO systems.

- We proposed just some possible options for the hybrid processing in both BS and MS, however, this is a field that is still in discussion. We want to invite the readers to propose their own hybrid precoders/combiners, and we hope that this text helps them in this process.

- Trying sub-connected architectures i.e., an RF chain is connected to a group of antennas. Therefore, the antenna array in the transmitter or receiver is built it using many antenna arrays (an array of arrays). Considering this kind of architectures, the matrices corresponding to the analog parts of the hybrid processing have zeros that represent the noconnection, which produces its design more challenging.

- The designing problem of the analog part of hybrid processing can be formulated as a selection problem. A group of fixed beams is defined for the precoder or combiner. This group is known as a codebook. Here two approaches can be researched, the first one is about finding an appropriate codebook design to face the adverse channel conditions presented in mmWave scenarios, and the second one is to propose a beam selection criterion. This last topic can be approached by tools of artificial intelligence field as deep learning, or reinforcement learning. 


\section{Bibliography}

[1] EL AYACH, O.; RAJAGOPAL, S.; ABU-SURRA, S.; PI, Z. ; HEATH, R. W.. Spatially sparse precoding in millimeter wave MIMO systems. IEEE transactions on wireless communications, 13(3):1499-1513, 2014.

[2] TORKILDSON, E.; SHELDON, C.; MADHOW, U. ; RODWELL, M.. Millimeter-wave spatial multiplexing in an indoor environment. In: GLOBECOM WORKSHOPS, 2009 IEEE, p. 1-6. IEEE, 2009.

[3] WANG, J.. Beam codebook based beamforming protocol for multiGbps millimeter-wave WPAN systems. IEEE Journal on Selected Areas in Communications, 27(8), 2009.

[4] EL AYACH, O.; HEATH, R. W.; ABU-SURRA, S.; RAJAGOPAL, S. ; PI, Z.. Low complexity precoding for large millimeter wave MIMO systems. In: COMMUNICATIONS (ICC), 2012 IEEE INTERNATIONAL CONFERENCE ON, p. 3724-3729. IEEE, 2012.

[5] EL AYACH, O.; HEATH, R. W.; ABU-SURRA, S.; RAJAGOPAL, S. ; PI, Z.. The capacity optimality of beam steering in large millimeter wave MIMO systems. In: SIGNAL PROCESSING ADVANCES IN WIRELESS COMMUNICATIONS (SPAWC), 2012 IEEE 13TH INTERNATIONAL WORKSHOP ON, p. 100-104. IEEE, 2012.

[6] LOTA, J.; SUN, S.; RAPPAPORT, T. S. ; DEMOSTHENOUS, A.. 5G Uniform Linear Arrays with Beamforming and Spatial Multiplexing at 28, 37, 64, and $71 \mathrm{GHz}$ for Outdoor Urban Communication: A Two-Level Approach. IEEE Transactions on Vehicular Technology, 66(11):9972-9985, 2017.

[7] MOLISCH, A. F.; RATNAM, V. V.; HAN, S.; LI, Z.; NGUYEN, S. L. H.; LI, L. ; HANEDA, K.. Hybrid beamforming for massive MIMO: A survey. IEEE Communications Magazine, 55(9):134-141, 2017.

[8] BOGALE, T. E.; LE, L. B.; HAGHIGHAT, A. ; VANDENDORPE, L.. On the number of rf chains and phase shifters, and scheduling design with hybrid analog-digital beamforming. IEEE Transactions on Wireless Communications, 15(5):3311-3326, 2016. 
[16] KWON, G.; PARK, H.. A joint scheduling and millimeter wave hybrid beamforming system with partial side information. In: COMMUNICATIONS (ICC), 2016 IEEE INTERNATIONAL CONFERENCE ON, p. 1-6. IEEE, 2016.

[17] SONG, J.; CHOI, J. ; LOVE, D. J.. Common codebook millimeter wave beam design: Designing beams for both sounding and communication with uniform planar arrays. IEEE Transactions on Communications, 65(4):1859-1872, 2017.

[18] AlKhAteeb, A.; El AYACH, O.; LeUS, G. ; HeATH, R. W.. Hybrid precoding for millimeter wave cellular systems with partial channel knowledge. In: INFORMATION THEORY AND APPLICATIONS WORKSHOP (ITA), 2013, p. 1-5. IEEE, 2013.

[19] LEE, J.; GIL, G.-T. ; LEE, Y. H.. Channel estimation via orthogonal matching pursuit for hybrid MIMO systems in millimeter wave communications. IEEE Transactions on Communications, 64(6):23702386, 2016.

[20] MIRZA, J.; ALI, B.; NAQVI, S. S. ; SALEEM, S.. Hybrid precoding via successive refinement for millimeter wave MIMO communication systems. IEEE Communications Letters, 21(5):991-994, 2017.

[21] LI, A.; MASOUROS, C.. Hybrid precoding and combining design for millimeter-wave multi-user MIMO based on SVD. In: COMMUNICATIONS (ICC), 2017 IEEE INTERNATIONAL CONFERENCE ON, p. 1-6. IEEE, 2017.

[23] BOGALE, T. E.; LE, L. B.. Beamforming for multiuser massive MIMO systems: Digital versus hybrid analog-digital. arXiv preprint arXiv:1407.0446, 2014.

[24] HU, C.; LIU, J.; LIAO, X.; LIU, Y. ; WANG, J.. A novel equivalent baseband channel of hybrid beamforming in massive multiuser MIMO systems. IEEE Commun. Lett., vol. PP, (99):1-1, 2017.

[25] ZU, K.; DE LAMARE, R. C. ; HAARDT, M.. Generalized design of low-complexity block diagonalization type precoding algorithms for multiuser MIMO systems. IEEE Transactions on Communications, 61(10):4232-4242, 2013. 
[26] PI, Z.. Optimal transmitter beamforming with per-antenna power constraints. In: COMMUNICATIONS (ICC), 2012 IEEE INTERNATIONAL CONFERENCE ON, p. 3779-3784. IEEE, 2012.

[29] ORTEGA, Á. J.; SAMPAIO-NETO, R. ; DAVID, R. P.. Hybrid precoded index modulation in downlink mmwave MU-MIMO systems. In: 2019 INTERNATIONAL CONFERENCE ON COMPUTING, NETWORKING AND COMMUNICATIONS (ICNC), p. 329-333. IEEE, 2019.

[30] SCAGLIONE, A.; STOICA, P.; BARBAROSSA, S.; GIANNAKIS, G. B. ; SAMPATH, H.. Optimal designs for space-time linear precoders and decoders. IEEE Transactions on Signal Processing, 50(5):1051-1064, 2002.

[31] JOHAM, M.; UTSCHICK, W. ; NOSSEK, J. A.. Linear transmit processing in MIMO communications systems. IEEE Transactions on signal Processing, 53(8):2700-2712, 2005.

[32] IOUSHUA, S. S.; ELDAR, Y. C.. Hybrid analog-digital beamforming for massive MIMO systems. arXiv preprint arXiv:1712.03485, 2017.

[33] MÉNDEZ-RIAL, R.; RUSU, C.; PRELCIC, N. G.; ALKHATEEB, A. ; HEATH JR, R. W.. Hybrid MIMO architectures for millimeter wave communications: Phase shifters or switches? IEEE Access, 4(8):247-267, 2016.

[34] KIM, M.; LEE, Y. H.. Mse-based hybrid RF/baseband processing for millimeter-wave communication systems in MIMO interference channels. IEEE Transactions on Vehicular Technology, 64(6):27142720, 2015.

[35] YU, X.; SHEN, J.-C.; ZHANG, J. ; LETAIEF, K. B.. Alternating minimization algorithms for hybrid precoding in millimeter wave MIMO systems. J. Sel. Topics Signal Processing, 10(3):485-500, 2016.

[36] COVER, T. M.; THOMAS, J. A.. Elements of information theory. John Wiley \& Sons, 2012.

[37] SMITH, P. J.; SENANAYAKE, R. ; DMOCHOWSKI, P. A.. How accurate is your gaussian/gamma approximation? IEEE Wireless Communications Letters, 7(5):804-807, 2018. 
[38] STAVRIDIS, A.; BASNAYAKA, D.; DI RENZO, M. ; HAAS, H.. Average bit error probability of receive-spatial modulation using zeroforcing precoding. In: 2014 IEEE 19TH INTERNATIONAL WORKSHOP ON COMPUTER AIDED MODELING AND DESIGN OF COMMUNICATION LINKS AND NETWORKS (CAMAD), p. 390-394. IEEE, 2014.

[39] ORTEGA, A. J.; SAMPAIO-NeTO, R. ; PEREIRA, R. D.. On hybrid precoder/combiner for downlink mmWave massive MU-MIMO systems. Preprint Researchgate DOI: 10.13140/RG.2.2.36339.58405, 2019.

[40] PÉREZ PAlOMAR, D.; RODRÍGUeZ FONOLlOSA, J.. Practical algorithms for a family of waterfilling solutions. 2005.

[41] GUO, R.; CAI, Y.; SHI, Q.; ZHAO, M. ; CHAMPAGNE, B.. Joint design of beam selection and precoding for mmwave MU-MIMO systems with lens antenna array. In: 2017 IEEE 28TH ANNUAL INTERNATIONAL SYMPOSIUM ON PERSONAL, INDOOR, AND MOBILE RADIO COMMUNICATIONS (PIMRC), p. 1-5. IEEE, 2017.

[42] RAGHAVAN, V.; SUBRAMANIAN, S.; CEZANNE, J.; SAMPATH, A.; KOYMEN, O. H. ; LI, J.. Single-user versus multi-user precoding for millimeter wave MIMO systems. IEEE Journal on Selected Areas in Communications, 35(6):1387-1401, 2017.

[43] EL AYACH, O.; HEATH, R. W.; ABU-SURRA, S.; RAJAGOPAL, S. ; PI, Z.. The capacity optimality of beam steering in large millimeter wave MIMO systems. In: 2012 IEEE 13TH INTERNATIONAL WORKSHOP ON SIGNAL PROCESSING ADVANCES IN WIRELESS COMMUNICATIONS (SPAWC), p. 100-104. IEEE, 2012.

[44] SALEH, A. A.; VALENZUELA, R.. A statistical model for indoor multipath propagation. IEEE Journal on selected areas in communications, 5(2):128-137, 1987.

[45] ORTEGA, Á. J.; SAMPAIO-NETO, R. ; DAVID, R. P.. Approximate expressions for BER performance in downlink mmwave MUMIMO hybrid systems. In: 2019 16TH INTERNATIONAL SYMPOSIUM ON WIRELESS COMMUNICATION SYSTEMS (ISWCS). IEEE, 2019.

[46] AlKhATEeB, A.; El AYACH, O.; LeUS, G. ; HEATH, R. W.. Channel estimation and hybrid precoding for millimeter wave cellular systems. IEEE Journal of Selected Topics in Signal Processing, 8(5):831846, 2014. 
[47] ALKHATEEB, A.; EL AYACH, O.; LEUS, G. ; HEATH, R. W.. Single-sided adaptive estimation of multi-path millimeter wave channels. In: 2014 IEEE 15TH INTERNATIONAL WORKSHOP ON SIGNAL PROCESSING ADVANCES IN WIRELESS COMMUNICATIONS (SPAWC), p. 125-129. IEEE, 2014.

[48] BAJWA, W. U.; HAUPT, J.; SAYEED, A. M. ; NOWAK, R.. Compressed channel sensing: A new approach to estimating sparse multipath channels. Proceedings of the IEEE, 98(6):1058-1076, 2010.

[49] THOMAS, C. K.; SLOCK, D.. Deterministic annealing for hybrid beamforming design in multi-cell MU-MIMO systems. In: 2018 IEEE 19TH INTERNATIONAL WORKSHOP ON SIGNAL PROCESSING ADVANCES IN WIRELESS COMMUNICATIONS (SPAWC), p. 1-5. IEEE, 2018.

[50] THOMAS, C. K.; SLOCK, D.. Mixed time scale weighted sum rate maximization for hybrid beamforming in multi-cell MU-MIMO systems. In: 2017 IEEE GLOBECOM WORKSHOPS (GC WKSHPS), p. 1-6. IEEE, 2017.

[51] PARK, S.; PARK, J.; YAZDAN, A. ; HEATH, R. W.. Exploiting spatial channel covariance for hybrid precoding in massive MIMO systems. IEEE Transactions on Signal Processing, 65(14):3818-3832, 2017.

[52] REY, F.; LAMARCA, M. ; VÁZQUEZ, G.. Transmit filter optimization based on partial csi knowledge for wireless applications. In: IEEE INTERNATIONAL CONFERENCE ON COMMUNICATIONS, 2003. ICC'03., volumen 4, p. 2567-2571. Citeseer, 2003. 
A

\section{Appendix}

\section{A.1}

\section{General linear constrained MMSE Precoding}

Here we consider that the MIMO system consists of a linear precoder with precoding matrix $\mathbf{F}$ at the transmitter, which is constrained by a complex matrix $\mathbf{B}$. The output signal of the detector is described by

$$
\tilde{\mathbf{x}}=\mathbf{H B F} \mathbf{x}+\mathbf{n},
$$

where $\mathbf{H}$ is the MIMO channel, $\mathbf{x}$ is the input signal and $\mathbf{n}$ is the additive Gaussian noise vector. The input signal $\mathbf{x}$ has covariance matrix $\mathbb{E}\left[\mathbf{x x}^{H}\right]=\mathbf{R}_{\mathbf{x}}$. The noise $\mathbf{n}$ has covariance matrix $\mathbb{E}\left[\mathbf{n n}^{H}\right]=\mathbf{R}_{\mathbf{n}}$. Because the transmit energy is constrained, the received signal is scaled by a factor $\beta$ at the receiver, which is part of the optimization.

The MMSE precoder is obtained from the following optimization problem

$$
\begin{array}{r}
\left\{\mathbf{F}_{M M S E}, \beta_{M M S E}\right\}=\underset{\mathbf{F}, \beta}{\arg \min } \mathbb{E}\left[\|\mathbf{x}-\beta \tilde{\mathbf{x}}\|^{2}\right] \\
\text { s.t. } \mathbb{E}\left[\|\mathbf{B F \mathbf { x }}\|^{2}\right]=\operatorname{tr}\left\{\mathbf{A F R}_{x} \mathbf{F}^{H}\right\} \leq E_{T},
\end{array}
$$

where $\mathbf{A}=\mathbf{B}^{H} \mathbf{B}$ and $E_{T}$ is the total transmit energy. The necessary conditions for the precoder filter $\mathbf{F}$ and weight $\beta \in \mathbb{R}_{+}$can be found by constructing the Lagrangian function

$$
\begin{aligned}
L(\mathbf{F}, \beta, \lambda)= & \mathbb{E}\left[\|\mathbf{x}-\beta \tilde{\mathbf{x}}\|^{2}\right]+\lambda\left(\operatorname{tr}\left\{\mathbf{A F R}_{x} \mathbf{F}\right\}-E_{T}\right) \\
= & \operatorname{tr}\left\{\mathbf{R}_{x}\right\}-\beta \mathbb{E}\left[\mathbf{x}^{H} \overline{\mathbf{H}} \mathbf{F} \mathbf{x}\right]-\beta \mathbb{E}\left[\mathbf{x}^{H} \mathbf{F}^{H} \overline{\mathbf{H}}^{H} \mathbf{x}\right] \\
& +\beta^{2} \mathbb{E}\left[\mathbf{x}^{H} \mathbf{F}^{H} \overline{\mathbf{H}}^{H} \overline{\mathbf{H}} \mathbf{F} \mathbf{x}\right]+\beta^{2} \mathbb{E}\left[\mathbf{n}^{H} \mathbf{n}\right] \\
& +\lambda\left(\operatorname{tr}\left\{\mathbf{A} \mathbf{F} \mathbf{R}_{x} \mathbf{F}^{H}\right\}-E_{T}\right) .
\end{aligned}
$$

where $\overline{\mathbf{H}}=\mathbf{H B}$ and $\lambda$ is the Lagrangian multiplier. By making use of the trace operator and its properties, the Lagrangian function can be rewritten as 
follows

$$
\begin{aligned}
L(\mathbf{F}, \beta, \lambda)= & \operatorname{tr}\left\{\mathbf{R}_{x}\right\}-\beta \operatorname{tr}\left\{\overline{\mathbf{H}} \mathbf{F} \mathbf{R}_{x}\right\}-\beta \operatorname{tr}\left\{\overline{\mathbf{H}}^{H} \mathbf{R}_{x} \mathbf{F}^{H}\right\} \\
& +\beta^{2} \operatorname{tr}\left\{\overline{\mathbf{H}}^{H} \overline{\mathbf{H}} \mathbf{F} \mathbf{R}_{x} \mathbf{F}^{H}\right\}+\beta^{2} \operatorname{tr}\left\{\mathbf{R}_{n}\right\} \\
& +\lambda\left(\operatorname{tr}\left\{\mathbf{A F R}_{x} \mathbf{F}^{H}\right\}-E_{T}\right) .
\end{aligned}
$$

Then, setting its derivatives to zero

$$
\begin{aligned}
& \frac{\partial L(\mathbf{F}, \beta, \lambda)}{\partial \mathbf{F}^{*}}=2 \beta^{2} \overline{\mathbf{H}}^{H} \overline{\mathbf{H}} \mathbf{F} \mathbf{R}_{x}-2 \beta \overline{\mathbf{H}}^{H} \mathbf{R}_{x}+2 \lambda \mathbf{A} \mathbf{F} \mathbf{R}_{x}=\mathbf{0} \\
& \frac{\partial L(\mathbf{F}, \beta, \lambda)}{\partial \beta}=-\operatorname{tr}\left\{\overline{\mathbf{H}} \mathbf{F} \mathbf{R}_{x}\right\}-\operatorname{tr}\left\{\overline{\mathbf{H}}^{H} \mathbf{R}_{x} \mathbf{F}^{H}\right\} \\
&+2 \beta \operatorname{tr}\left\{\overline{\mathbf{H}}^{H} \overline{\mathbf{H}} \mathbf{F} \mathbf{R}_{x} \mathbf{F}^{H}\right\}+2 \beta \operatorname{tr}\left\{\mathbf{R}_{n}\right\} \\
&=-2 \operatorname{tr}\left\{\overline{\mathbf{H}}^{H} \mathbf{R}_{x} \mathbf{F}^{H}\right\} \\
&+2 \beta \operatorname{tr}\left\{\overline{\mathbf{H}}^{H} \overline{\mathbf{H}} \mathbf{F} \mathbf{R}_{x} \mathbf{F}^{H}\right\}+2 \beta \operatorname{tr}\left\{\mathbf{R}_{n}\right\}=0
\end{aligned}
$$

We obtain from (A-5) that

$$
\beta^{-1} \overline{\mathbf{H}}^{H}=\left(\overline{\mathbf{H}}^{H} \overline{\mathbf{H}}+\lambda \beta^{-2} \mathbf{A}\right) \mathbf{F}
$$

thus resulting for the precoder filter the structure

$$
\mathbf{F}=\beta^{-1}\left(\overline{\mathbf{H}}^{H} \overline{\mathbf{H}}+\lambda \beta^{-2} \mathbf{A}\right)^{-1} \overline{\mathbf{H}}^{H}
$$

Multiplying from the right $2 \beta \mathbf{R}_{x} \mathbf{F}^{H}$ in (A-7) and using the trace operator yields

$$
\begin{aligned}
2 \operatorname{tr}\left\{\overline{\mathbf{H}}^{H} \mathbf{R}_{x} \mathbf{F}^{H}\right\} & =2 \beta \operatorname{tr}\left\{\left(\overline{\mathbf{H}}^{H} \overline{\mathbf{H}}+\lambda \beta^{-2} \mathbf{A}\right) \mathbf{F} \mathbf{R}_{x} \mathbf{F}^{H}\right\} \\
& =2 \beta \operatorname{tr}\left\{\overline{\mathbf{H}}^{H} \overline{\mathbf{H}} \mathbf{F} \mathbf{R}_{x} \mathbf{F}^{H}\right\}+2 \lambda \beta^{-1} \operatorname{tr}\left\{\mathbf{A} \mathbf{F} \mathbf{R}_{x} \mathbf{F}^{H}\right\}
\end{aligned}
$$

Using (A-6) in (A-9) we have that

$$
\lambda \beta^{-2}=\frac{\operatorname{tr}\left\{\mathbf{R}_{n}\right\}}{\operatorname{tr}\left\{\mathbf{A F R}_{x} \mathbf{F}^{H}\right\}}
$$

Equation (A-10) evidences that $\lambda$ takes values grater than zero, and therefore the solution of $\mathbf{F}$ is in the border of the admissible search space, meaning that equality holds in (A-2), $\operatorname{tr}\left\{\mathbf{A F R}_{x} \mathbf{F}^{H}\right\}=E_{T}$, and 


$$
\lambda \beta^{-2}=\frac{\operatorname{tr}\left\{\mathbf{R}_{n}\right\}}{E_{T}}
$$

Substituting (A-11) in (A-8), we obtain

$$
\mathbf{F}=\beta^{-1}\left(\overline{\mathbf{H}}^{H} \overline{\mathbf{H}}+\frac{\operatorname{tr}\left\{\mathbf{R}_{n}\right\}}{E_{T}} \mathbf{A}\right)^{-1} \overline{\mathbf{H}}^{H}
$$

and the equality constraint

$$
\beta^{-2} \operatorname{tr}\left\{\mathbf{A}\left(\overline{\mathbf{H}}^{H} \overline{\mathbf{H}}+\frac{\operatorname{tr}\left\{\mathbf{R}_{n}\right\} \mathbf{A}}{E_{T}}\right)^{-1} \overline{\mathbf{H}}^{H} \mathbf{R}_{x} \overline{\mathbf{H}}\left(\overline{\mathbf{H}}^{H} \overline{\mathbf{H}}+\frac{\operatorname{tr}\left\{\mathbf{R}_{n}\right\} \mathbf{A}}{E_{T}}\right)^{-1}\right\}=E_{T}
$$

yields the expression for the scaling factor $\beta^{-1}$ in $(\mathrm{A}-12)$

$$
\beta_{M M S E}^{-1}=\sqrt{\frac{E_{T}}{\operatorname{tr}\left\{\mathbf{A}\left(\overline{\mathbf{H}}^{H} \overline{\mathbf{H}}+\frac{\operatorname{tr}\left\{\mathbf{R}_{n}\right\} \mathbf{A}}{E_{T}}\right)^{-1} \overline{\mathbf{H}}^{H} \mathbf{R}_{x} \overline{\mathbf{H}}\left(\overline{\mathbf{H}}^{H} \overline{\mathbf{H}}+\frac{\operatorname{tr}\left\{\mathbf{R}_{n}\right\} \mathbf{A}}{E_{T}}\right)^{-1}\right\}}}
$$

Therefore, the general linear constrained MMSE precoding is given by

$$
\begin{aligned}
\mathbf{F}_{M M S E}= & \sqrt{\frac{E_{T}}{\operatorname{tr}\left\{\mathbf{A}\left(\overline{\mathbf{H}}^{H} \overline{\mathbf{H}}+\frac{\operatorname{tr}\left\{\mathbf{R}_{n}\right\} \mathbf{A}}{E_{T}}\right)^{-1} \overline{\mathbf{H}}^{H} \mathbf{R}_{x} \overline{\mathbf{H}}\left(\overline{\mathbf{H}}{ }^{H} \overline{\mathbf{H}}+\frac{\operatorname{tr}\left\{\mathbf{R}_{n}\right\} \mathbf{A}}{E_{T}}\right)^{-1}\right\}}} \\
& \times\left(\overline{\mathbf{H}}^{H} \overline{\mathbf{H}}+\frac{\operatorname{tr}\left\{\mathbf{R}_{n}\right\}}{E_{T}} \mathbf{A}\right)^{-1} \overline{\mathbf{H}}^{H}
\end{aligned}
$$

